\title{
Locality and the Structure of Particle States
}

\author{
Detlev Buchholz ${ }^{1}$ and Klaus Fredenhagen ${ }^{2}$ \\ 1 II. Institut für Theoretische Physik der Universität Hamburg, D-2000 Hamburg, Federal Republic \\ of Germany \\ 2 Fakultät für Physik der Universität Freiburg, D-7800 Freiburg, Federal Republic of Germany
}

\begin{abstract}
Starting from the principle of locality of observables we derive localization properties of massive particle states which hold in all models of relativistic quantum theory, including gauge theories. It turns out that particles may always be regarded as well localized distributions of matter, although their mathematical description might require the introduction of non-local (unobservable) fields, which are assigned to infinite string-like regions. In spite of the non-locality of these fields one can show that such particles obey Bose- or Fermi (para) statistics, that to each particle there exists an antiparticle and that collision states of particles exist. A selfcontained exposition of the underlying physical ideas is given in the Introduction, and some perspectives for the structure of field-theoretic models arising from our analysis are discussed in the Conclusions.
\end{abstract}

\section{Introduction}

Many qualitative features of particle physics, such as the existence of antiparticles, the phenomenon of particle statistics and the formation of asymptotic particle configurations in collision processes have found a theoretical explanation based on the assumption that particle states are well localized excitations of some vacuum state. In the Wightman-framework of quantum field theory one converts this physical picture into the hypothesis that particle states can be constructed by applying local field operators to the vector representing the vacuum [2]. A physically more transparent formulation has been given by Doplicher, Haag and Roberts $[3,4]$. Using only the concept of local observables, these authors expressed in an algebraic setting the assumption that particle states cannot be distinguished from the vacuum by measurements in the spacelike complement of sufficiently large, but bounded regions of Minkowski space.

These descriptions of localization properties are perfectly adequate for charged particles in theories with a global gauge symmetry. But it is well known that they cannot be applied to particles carrying an electric charge: since it is possible to 
determine the electric charge by measuring the total electric flux through an arbitrarily large sphere surrounding a particle, states with an electric charge can be discriminated from the vacuum in the causal complement of any bounded region $[3,5]$. Such charges which can be measured at spacelike infinity appear typically in gauge field theories, and to fix terminology we will call them gaugecharges.

The example of the electric charge might suggest that gauge-charges are always connected with long-range forces and the appearance of massless particles. Indeed, this is generally true in Abelian gauge theories [1,6]. But in non-Abelian gauge theories, such as quantum chromodynamics, the argument fails, and the work on lattice gauge theories indicates that states carrying a gauge-charge may well exist in the absence of massless particles ${ }^{1}$. We shall also see from our general structural analysis that massive particle states might have weaker localization properties than normally assumed. This would admit gauge-charges even in purely massive theories.

In the traditional, perturbative treatment of gauge theories one uses local but unobservable fields revealing the local gauge symmetry and acting on a Hilbert space of indefinite metric. This approach has been axiomatized by various authors (see e.g. [5]), but unfortunately it has not provided much insight into the structural properties of charged physical states beyond the range of perturbation theory. The reason is that the basic fields generate a set of unphysical states by which the charged physical states must be approximated. But this approximation requires a topology in the indefinite metric space, and since the choice of this topology is so far highly ambiguous, one has no effective control on the structure of the limiting states one is interested in.

This unsatisfactory situation has stimulated us to reconsider the localization properties of particles in the Haag-Kastler framework of local quantum theory [7]. In this setting one regards the algebraic properties of the local observables as the fundamental structure which embodies the relevant information about the physical states. Therefore, if an algebra of local observables is given, it must in principle be possible to derive the properties of the corresponding physical states without introducing unobservable fields ab initio.

This general program resembles the procedure which one applies in constructive gauge field theory. There, the existing algorithms, such as the lattice approach or the methods using functional integrals are designed to define the local, gauge invariant observables of the theory in the representation which is induced by the vacuum state; unobservable fields merely appear as integration variables. Since the vacuum representation of the observables covers only the coherent subspace of states carrying the charge quantum numbers of the vacuum, one must extract in a second step from properties of suitable observables (such as the Wilson loops

1 The gluons which appear in a perturbative treatment of quantum chromodynamics are usually treated as massless objects. But they are not invariant under local gauge transformations, so they are not real particles contributing to the physical energy-momentum spectrum. We use here the term particle only for physical states appearing asymptotically in collision processes 
[8]) the information of whether there exist also charged states in the theory and what their properties are. This procedure is analogous to the general ideas of Haag and Kastler, and one may in fact regard the gauge theories as prototypes of theories of local observables.

The construction of the physical Hilbert space is accomplished in this setting in a manner which is perhaps not so familiar, therefore we recall here the basic ideas. Any physical state corresponds to some positive linear functional $\omega$ over an algebra of observables $\mathfrak{A}$. So in a first step one has to select all functionals exhibiting properties which are distinctive of particle states. This step is perhaps the most subtle one in the whole analysis because there the physical idea of a particle has to be converted into appropriate mathematical constraints. But once such a set of particle states $\omega$ has been chosen there exist standard methods for the construction of the physical Hilbert space. One uses the basic fact (GNS-construction [9]) that to each state $\omega$ there exists a representation $\pi$ of the algebra $\mathfrak{A}$ on some Hilbert space $\mathscr{H}$, in which $\omega$ is represented by a unit vector $\Psi$ and the observables $A \in \mathfrak{A}$ by operators $\pi(A)$ such that

$$
\omega(A)=(\Psi, \pi(A) \Psi) .
$$

The vectors in $\mathscr{H}$ are to be interpreted as those physical states which arise from the ensemble described by $\Psi$ due to the action of local observables. So in particular they carry the same charge quantum numbers as $\Psi$.

Of course equivalent representations of $\mathfrak{A}$ describe identical sets of states. Therefore one combines the irreducible representations into equivalence classes, called sectors. If there are superselection rules in a model there exist several such sectors, and one may regard the labels distinguishing them as charge quantum numbers. This fundamental concept of charge applies also to superselection rules which are related to the Casimir operators of non-Abelian gauge groups or to topological charges which cannot be derived from an observable current, and it is therefore perfectly adequate for a structural analysis of gauge theories. Having determined the sectors of a theory, one can then construct a representation of the algebra of observables on a global physical Hilbert space by picking representations from each sector and taking their direct sum.

The above considerations show that even if charge carrying fields are not at one's disposal it is possible to determine the physical Hilbert space and its superselection structure, provided one has selected by some reasonable criterion all states of physical interest. There are two such selection criteria which have been used in the literature. One criterion which we have already mentioned is due to Doplicher, Haag and Roberts [3]. These authors select the states $\omega$ of interest in particle physics by the assumption that they become indistinguishable from the vacuum for observations at spacelike infinity. This excludes from the outset states carrying a gauge-charge as defined above.

Such states are, however, covered by a selection criterion of Borchers [10], who uses momentum space properties of states in order to distinguish the physically relevant representations. Following Borchers, we select the representations of interest in particle physics by the condition that the space-time translations are generated by energy-momentum operators satisfying the spectrum condition. 
One might argue that in representations describing particle states the generators of the Lorentz-transformations should also exist as well-defined operators. However, this is in general too restrictive an assumption because it requires localization properties of states which are not always present. For example, the charged sectors in quantum electrodynamics are not Lorentz-covariant [11], so one must dispense with this assumption in a general selection criterion.

As was already indicated, we will restrict in the present paper our attention to models describing only massive particles. (For an analysis of the physical state space of quantum electrodynamics see [12].) Moreover, we assume that the models under consideration have an asymptotic particle interpretation in the sense of scattering theory (see for example [13]). This assertion is not used in a technical sense, but its obvious physical content provides a heuristic background for various steps in our analysis.

Since in a particle theory all states of interest should be composed of asymptotic configurations of single particle states, it is natural to begin these investigations with a detailed analysis of representations $\pi$ of $\mathfrak{A}$ in which states of just one particle appear. We distinguish these representations by the fact that the corresponding representation space $\mathscr{H}$ contains a subspace $\mathscr{H}^{(1)}$, on which the relation $P_{0}=\left(\mathbf{P}^{2}+m^{2}\right)^{1 / 2}$ relating the energy and momentum of a particle of mass $m$ holds. Restricting our attention to charged particles we assume furthermore that the vectors in $\mathscr{H}^{(1)}$ are the states of lowest mass in $\mathscr{H}$, and taking into account that there are no massless particles in the model, we require that the energy-momentum spectrum of the vectors in $\mathscr{H}^{(1)}$ is isolated from the rest of the spectrum by a mass gap; this means that $P_{0} \geqq\left(\mathbf{P}^{2}+M^{2}\right)^{1 / 2}$ for some $M>m$ on the orthogonal complement of the single particle space $\mathscr{H}^{(1)}$.

The existence of such representations of $\mathfrak{U}$ is clearly a necessary condition for a model to have a conventional particle interpretation. However, since we did not require Lorentz covariance it might appear artificial to characterize the vectors in $\mathscr{H}^{(1)}$ by Lorentz-invariant subsets of the energy-momentum spectrum. But it follows already from locality of the observables that the lower boundary of the energy-momentum spectrum is a Lorentz-invariant set in all representations satisfying the selection criterion of Borchers [15]. In Sect. 2 we will sketch the argument leading to this result; moreover we will prove that the spectrum of the spatial momentum operators $\mathbf{P}$ is continuous with respect to Lebesgue-measure, which is a well known fact in the presence of Lorentz transformations [14]. So also under the present general conditions one may identify the vectors in $\mathscr{H}^{(1)}$ with square integrable wavefunctions $\varphi(\mathbf{p})$ (if one disregards internal degrees of freedom).

Newton and Wigner have discussed the concept of localization of a single particle state in terms of such wavefunctions [16]. It is, however, not clear how the Newton-Wigner position operator is related to the local observables in $\mathfrak{A}$. Therefore we will exploit here the local structure of observables directly for the identification of well localized single particle states in $\mathscr{H}^{(1)}$. This will be done in Sect. 3.

Thinking in terms of measurements, it is quite plausible how these states are constructed. First one chooses a vector $\Phi_{\mathbf{q}} \in \mathscr{H}^{(1)}$ with a momentum space wavefunction which has support in a small neighbourhood of some point $\mathbf{q}$. So $\Phi_{\mathbf{q}}$ 
describes an ensemble of particles with relatively sharp momentum, but no specific localization properties. Then one picks an observable $A \in \mathfrak{A}$ which is localized in some bounded space-time region $\mathcal{O}$ and applies it to $\Phi_{\mathbf{q}}$. The resulting vector $\pi(A) \Phi_{\mathbf{q}}$ should describe the effects of an operation in the region $\mathcal{O}$ on an ensemble of single particle states with momentum $\mathbf{q}$, which are expected to be of the following nature: some of the particles in the ensemble will come close to the region $\mathcal{O}$ and interact with the observable $A$, and this will cause a change of their momentum. On the other hand, the presence of $A$ can have no effect on particles which keep a sufficient distance from $\mathcal{O}$, so their momentum remains unchanged. It will also happen with a certain probability that additional particles are created by the action of $A$, resulting in a change of the total mass; so all these typical events can be distinguished by their characteristic energy-momentum content.

One can choose now a function $g(P)$ of the energy and momentum operator which vanishes on single-particle states with momentum about $\mathbf{q}$ and on all multi-particle states. According to the above remarks one then expects that the vector $g(P) \cdot \pi(A) \Phi_{\mathbf{q}}$ describes an ensemble of particles coming close to the region $\mathcal{O}$. Of course, the action of $g(P)$ will destroy again some of the information on the localization properties because of the uncertainty-principle. But in view of the mass gap between the single- and multi-particle states, there exist smooth functions $g(P)$ with the above mentioned properties, for which this effect is small. So the vector $\Psi=g(P) \cdot \pi(A) \Phi_{\mathbf{q}}$ should describe a well localized single particle state.

A mathematical expression of this fact is that any translation $U(x)$ of $\Psi$ can be represented by the action of some suitable almost local operator $B_{x}$ on $\Psi$, i.e.

$$
U(x) \Psi=B_{x} \Psi \quad \text { and } \quad U(-x) \Psi=B_{x}^{*} \Psi
$$

This means that the translations $U(x)$ essentially become effective only in a bounded region, which one may interpret as the localization region of $\Psi$ if $x$ is small. (Actually we will prove a slightly weaker form of this relation; but with the same physical content.) So for the characterization of the localization properties of $\Psi$ no reference to a vacuum representation is necessary.

The existence of vectors in a representation $\pi$ with the property (1.2) has several significant consequences. First, it follows that the expectation values $(U(x) \Psi, \pi(A) U(x) \Psi)$ of local observables $A \in \mathfrak{A}$ converge rapidly to some positive, linear functional $\omega_{0}$ over $\mathfrak{A}$ if $x$ tends to spacelike infinity,

$$
\lim _{x}(U(x) \Psi, \pi(A) U(x) \Psi)=\omega_{0}(A),
$$

and $\omega_{0}$ exhibits all properties of a vacuum state. So the space-like asymptotic structure of $\Psi$ can be described in terms of a single vacuum, and $\Psi$ may be regarded as an excitation of it.

Second, it follows from relation (1.2) that it is impossible to determine the sector (the total charge) of $\Psi$ by measurements in the causal complement of certain string-like regions. More precisely, one can show that the representation $\pi$ of $\mathfrak{U}$ (which is induced by $\Psi$ ) and the vacuum representation $\pi_{0}$ are equivalent on the subalgebras $\mathfrak{A}\left(\mathscr{S}^{\prime}\right)$ of observables which are localized in the causal complement 
$\mathscr{S}^{\prime}$ of any given spacelike cone $\mathscr{S}^{2}$, i.e.

$$
\pi\left\lceil\mathfrak { U } ( \mathscr { S } ^ { \prime } ) \simeq \pi _ { 0 } \left\lceil\mathfrak{A}\left(\mathscr{S}^{\prime}\right)\right.\right.
$$

In the proof of this result one uses the fact that any translation of $\Psi$ can be decomposed into small shifts along an arbitrary path connecting the localization regions of $\Psi$ and of its translate. Now if one decomposes a translation $\mathbf{z}$ into $\mathbf{z}=\mathbf{x}+\mathbf{y}$ one gets from relation (1.2)

$$
U(\mathbf{z}) \Psi=U(\mathbf{y}) B_{\mathbf{x}} U(\mathbf{y})^{-1} \cdot B_{\mathbf{y}} \Psi
$$

and iterating this procedure it follows that any translation of $\Psi$ is induced by a product of almost local observables which are, roughly speaking, localized in a tube about the chosen path. But this implies that the effect of this translation cannot be observed in the causal complement of this tube because of locality. Therefore it is impossible to dinstinguish the states in the representations $\pi$ and $\pi_{0}$ by measurements in any region $\mathscr{S}^{\prime}$, because in the region $\mathscr{S}$ one can always bring in particles from spacelike infinity or remove them without changing the results of measurements in $\mathscr{S}^{\prime}$.

It is interesting to confront our results with the selection criterion of Doplicher, Haag and Roberts [3]. These authors conjectured that their criterion applies to all particle states in completely massive theories (hadronic physics). This would mean [3] that particle states induce representations which are equivalent to the vacuum representation on the larger subalgebras $\mathfrak{U}\left(\mathcal{O}^{\prime}\right)$ of observables in the causal complement of bounded regions $\mathcal{O}$,

$$
\pi \uparrow \mathfrak{U}\left(\mathcal{O}^{\prime}\right) \simeq \pi_{0} \uparrow \mathfrak{U}\left(\mathcal{O}^{\prime}\right)
$$

The picture of a particle which is implied by this stronger condition is the following one: a particle is a well localized system which is completely uncorrelated to the rest of the world in the sense that one may take it out of the world or add it without effecting measurements in the causal complement of its localization region. Now in physics charged particles can only be created in pairs, and it is known from gauge theories that this can happen with a high degree of coherence which becomes manifest in the form of a string (flux lines) connecting the particles. It is evident that particles sitting at the endpoints of such a string cannot be treated as isolated systems, and therefore the selection criterion of Doplicher, Haag and Roberts does not apply to them. In contrast, our general result (1.4) does not exclude the possibility of such particles in massive theories.

At present, there are no definite examples of massive theories in which the selection criterion of Doplicher, Haag and Roberts fails, although there are some candidates (see the Conclusions). It might therefore be worthwhile to outline the expected qualitative features of such models. We will use in this discussion the simple picture that single particle states carrying a gauge-charge are tied to an infinite string. In concrete examples this string can have a complicated structure, it may split or roughen, but this is not relevant here.

$2 \mathscr{S}$ may be thought of as a string which fattens 
There are two possibilities to be discussed. The first one is that the location of the string can be determined. Then its asymptotic direction is a classical observable (i.e. a superselection rule) because it cannot change under the influence of any local measurements due to locality. In this case there exists a continuum of inequivalent representations describing particles with different asymptotic stringdirections, so these representations are not equivalent to the vacuum representation on the algebras $\mathfrak{U}\left(\mathscr{S}^{\prime}\right)$ for arbitrary spacelike cones $\mathscr{S}$. But according to our general result this is not possible in massive particle theories, and, as a matter of fact, the above scenario describes exactly the situation which one finds in quantum electrodynamics [12].

The second possibility is that the asymptotic direction of the string cannot be observed, but its presence can be established nevertheless. In order to illustrate this we give an example: let us assume that one can determine whether strings cross a given surface an even or odd number of times. If one performs such a measurement on a sufficiently large sphere surrounding all particles in a given state, one should find the values 1 or -1 , depending on whether the sphere encloses an even or odd number of particles. So these states are separated by a (multiplicative) gaugecharge. But if one removes a part from the sphere and performs the measurement on the remaining surface one should find with equal probability the values 1 or -1 in any ensemble of particles (including the vacuum) if it is impossible to determine the direction of strings. This picture is only consistent if the vacuum is subject to large string-like fluctuations which close to loops, and which spread out in such a way that they cut large surfaces with boundary an even or odd number of times with equal probability. Moreover, string-like fluctuations which do not close to loops must be suppressed, because otherwise it would be impossible to discriminate states with an even or odd number of particles. So the characteristic features of models belonging to this second category are, that their vacuum states undergo fluctuations which make it impossible to determine the location of the string; but their exist global observables measuring the gauge-charge which are built up from local observables in a multiplicative way. In such models the selection criterion (1.6) of Doplicher, Haag and Roberts would be too restrictive, but the weaker condition (1.4) could still be used to select all particle states. We remark that the above picture seems to be realized in the (massive) Higgs-phase of certain lattice gauge theories (see the Conclusions).

Although the problem of the existence of such models is still open, we consider it interesting to study the consequences of the weaker localization properties (1.4) of a particle for the physical interpretation of a theory. Surprisingly enough practically all relevant empirical properties of a particle can be established starting from relation (1.4): each such particle has an antiparticle, it obeys (para-) Bose- or Fermistatistics, and scattering states of such particles exist. This is in our opinion a strong indication that there should be models of this type.

For the proof of these facts we have generalized the methods which were developed by Doplicher, Haag and Roberts for the analysis of representations satisfying their selection criterion. Whenever possible we will refer to their results, and the reader interested in technical details should have available a copy of the articles [3] and [4]. 
This part of our paper is organized as follows: in Sect. 4 we will show that there is a composition law of sectors which is an expression of the fact that charges can be added. Section 5 contains the construction of a field bundle which is a convenient substitute for the algebra of field operators in conventional field theory. However, in contrast to conventional field theory the "fields" to be found here are localized in spacelike cones $\mathscr{S}$. Commutation relations in the field bundle lead to the notion of statistics in this setting, and in Sect. 6 we briefly review the classification of possible particle statistics given in [3], which also applies here. This analysis requires the construction of charge conjugate sectors which can be performed as in [3] for sectors having normal (finite) satistics.

The pathological possibility of infinite statistics can be ruled out for massive particles, using the fact that single particle states are energy-momentum concentrated in the sense of relation (1.2). This has been proved in [17] for pure particle states, and we give here an extension of that argument to arbitrary single particle states. As a consequence each (factorial) representation in which single particle states appear is a multiple of an irreducible representation describing single particle states. This result is quite satisfactory from the point of view of physics, because it means that it is always possible to prepare by suitable measurements pure particle states, so all particles are elementary systems.

In Sect. 7 we will analyse the energy-momentum operators in the composed sectors and prove that there exists a unique choice for these operators having all properties expected on physical grounds. Moreover, we will introduce translations on the field bundle in a covariant manner, which is essential for the construction of scattering states in Sect. 8. A brief discussion of possible perspectives of our analysis for field theoretic models completes the paper.

We conclude this introduction with a list of assumptions and notations. We base our analysis on the properties of a net of algebras $\mathcal{O} \rightarrow \mathfrak{U}(\mathcal{O})$, which is indexed by the open, bounded regions $\mathcal{O} \subset \mathbb{R}^{4}$ of space and time. Each $\mathfrak{A}(\mathcal{O})$ is a $C^{*}$-algebra with unit which may be regarded as the algebra generated by the observables which can be measured in $\mathcal{O}$. The algebra of all local observables is denoted by $\mathfrak{A}$ and defined as the $C^{*}$-inductive limit of the local algebras,

$$
\mathfrak{A}=\bigcup_{\mathscr{O}}^{C^{*}} \mathfrak{A}(\mathcal{O}) .
$$

Similarly we set for unbounded regions $\mathscr{R} \subset \mathbb{R}^{4}$

$$
\mathfrak{U}(\mathscr{R})=\bigcup_{\mathcal{O} \subset \mathscr{R}}^{C^{*}} \mathfrak{U}(\mathcal{O}) .
$$

We assume that observables in spacelike separated regions $\mathcal{O}_{1}$ and $\mathcal{O}_{2}$ commute, i.e. locality holds,

$$
\mathfrak{U}\left(\mathcal{O}_{1}\right) \subset \mathfrak{U}^{c}\left(\mathcal{O}_{2}\right) \text { if } \quad \mathcal{O}_{1} \subset \mathcal{O}_{2}^{\prime} .
$$

Here $\mathfrak{A}^{c}(\mathcal{O})$ stands for the subalgebra of operators in $\mathfrak{A}$ which commute with all operators in $\mathfrak{A}(\mathcal{O})$. Moreover, we require that the group of space-time translations 
$x$ is implemented by automorphisms $\alpha_{x}$ of $\mathfrak{U}$ which respect the geometric structure of the net,

$$
\alpha_{x}(\mathfrak{A}(\mathcal{O})) \subset \mathfrak{U}(\mathcal{O}+x) .
$$

In Sect. 4 we will add to these basic postulates the assumption that the vacuum representation constructed in Sect. 3 fulfils the following weak duality condition:

$$
\pi_{0}\left(\mathfrak{A}^{c}(\mathscr{S})\right)^{-}=\pi_{0}(\mathfrak{U}(\mathscr{S}))^{\prime}
$$

for any spacelike cone $\mathscr{S}$. Here $\mathfrak{B}^{-}$denotes the weak closure of a subalgebra $\mathfrak{B} \subset \mathscr{B}\left(\mathscr{H}_{0}\right)$ and $\mathfrak{B}^{\prime}$ the commutant of $\mathfrak{B}$ in $\mathscr{B}\left(\mathscr{H}_{0}\right)$. Such duality assumptions are familiar from the work of Doplicher, Haag and Roberts [3]. Roughly speaking, they say that the vacuum cannot carry a non-Abelian charge. Although this sounds plausible, it would be desirable to understand the physical origin of this fact better. For algebras of observables which can be constructed from a set of local Wightmanfields, Bisognano and Wichmann [18] have established a somewhat different form of duality which would also be sufficient for our analysis.

\section{Energy and Momentum}

In this section we will discuss properties of the energy-momentum operators in representations $(\pi, \mathscr{H})$ of the algebra of observables $\mathfrak{U}$ which fulfil the selection criterion of Borchers. These representations are distinguished by the fact that the space-time translations $x=(t, \mathbf{x})$ are represented by strongly continuous, unitary operators $U(x)$ on $\mathscr{H}$ which implement the automorphisms $\alpha_{x}$,

$$
\pi\left(\alpha_{x}(A)\right)=U(x) \pi(A) U(x)^{-1} \quad \text { for } \quad A \in \mathfrak{A} .
$$

Moreover, the joint spectrum sp $\mathscr{U}$ of the generators $P=\left(P_{0}, \mathbf{P}\right)$ of $U(x)$ satisfies the relativistic spectrum condition ${ }^{3}$

$$
\operatorname{sp} \mathscr{U} \subset \bar{V}_{+}=\left\{p \in \mathbb{R}^{4}: p^{2} \geqq 0, p_{0} \geqq 0\right\} .
$$

As has been shown by Borchers [19], it is always possible under these conditions to choose the operators $U(x)$ in the weak closure of $\pi(\mathfrak{U})$,

$$
U(x) \in \pi(\mathfrak{U})^{-},
$$

and this choice is also the natural one, since then the generators $P$ can be interpreted as global observables, measuring the energy and momentum of the states in $\mathscr{H}$. Moreover, relation (2.3) implies that any subrepresentation of $\pi$ also has the properties listed above. So performing the central decomposition of $\pi$ we may concentrate in the following on factorial representations,

$$
\pi(\mathfrak{U})^{-} \cap \pi(\mathfrak{U})^{\prime}=\mathbb{C} \cdot 1,
$$

which means in physical terms, that we consider only states with definite charge quantum numbers. In principle one could decompose $\pi$ even into irreducible

3 We use the notation $p^{2}=p_{0}^{2}-|\mathbf{p}|^{2}$ and $|p|^{2}=p_{0}^{2}+|\mathbf{p}|^{2}$ 
representations, but such a decomposition has only a simple physical meaning if there exist vectors in $\mathscr{H}$ inducing pure states on $\mathfrak{A}$. (We postpone a discussion of this point to Sect. 6.)

In a factorial representation of $\mathfrak{A}$ the generators $P$ are fixed by condition (2.3) up to an arbitrary additive constant. It is convenient to adjust this constant such that the following more restrictive version of relation (2.2) holds:

$$
\left\{q \in \bar{V}_{+}: \operatorname{sp} \mathscr{U} \subset \bar{V}_{+}+q\right\}=\{0\},
$$

i.e. no lightcone inside $\bar{V}_{+}$contains all of $\operatorname{sp} \mathscr{U}$. In the following we call representations $(\pi, \mathscr{H})$ of $\mathfrak{A}$ which satisfy the conditions (2.1) up to (2.5) positive energy representations.

We will study now the consequences of locality for the structure of the energymomentum spectrum. This part of our analysis applies also to models describing massless particles, such as quantum electrodynamics. The first result, which we adapt from [15], restricts the admissible shape of the set $\operatorname{sp} \mathscr{U}$ in positive energy representations of $\mathfrak{U}$.

Proposition 2.1. i) The lower boundary of $\operatorname{sp} \mathscr{U}$ is a Lorentz-invariant set. ii) If one removes from $\mathrm{sp} \mathscr{U}$ its lower boundary and takes the closure, one obtains again a set with Lorentz-invariant lower boundary.

(The lower boundary of a closed set $K \subset \bar{V}_{+}$consists of all points in $K$ which can be connected with 0 by some curve in the complement of $K$.)

Proof. (We sketch here the essential steps of the argument in [15].) One starts from the observation that the shape of $\mathrm{sp} \mathscr{U}$ can be determined from properties of the vector-valued functions $x \rightarrow \pi\left(\alpha_{x}(A)\right) \Phi$, where $\Phi \in \mathscr{H}$ and $A \in \mathfrak{A}$. Clearly the supports of the Fourier transforms of these functions (which are defined in the sense of distributions) are contained $\operatorname{in}^{4} \operatorname{sp} \mathscr{U}-\operatorname{supp} \Phi$, where supp $\Phi$ is the spectral support of $\Phi$ relative to $P$.

On the other hand, for any open region $N$ intersecting with sp $\mathscr{U}-\operatorname{supp} \Phi$ there is a local observable $A$ and a testfunction $f$ with supp $\tilde{f} \subset N$ such that $\int d^{4} x f(x) \pi\left(\alpha_{x}(A)\right) \Phi \neq 0$. This can be seen as follows: for a fixed testfunction $f$ the integral $B \rightarrow \int d^{4} x f(x) U(x) B U(x)^{-1}, B \in \mathscr{B}(\mathscr{H})$, defines an ultraweakly continuous mapping on $\mathscr{B}(\mathscr{H})$. Hence if $\int d^{4} x f(x) \pi\left(\alpha_{x}(A)\right) \Phi=0$ for all local observables $A$, it follows that also $\int d^{4} x f(x) E\left(\Delta_{1}\right) \pi\left(\alpha_{x}(A)\right) E\left(\Delta_{2}\right) \Phi=0$, where $E\left(\Delta_{i}\right) \in \pi(\mathfrak{L})^{-}$are the spectral projections of $P$ attached to Borel sets $\Delta_{i} \subset \mathbb{R}^{4}, i=1,2$. Now given an open set $N$ intersecting with sp $\mathscr{U}-\operatorname{supp} \Phi$ we can find open sets $\Delta_{1}, \Delta_{2}$ intersecting with sp $\mathscr{U}$ and supp $\Phi$, respectively, such that the closure of $\Delta_{1}-\Delta_{2}$ is contained in $N$. Then the integral $\int d^{4} x f(x) E\left(\Delta_{1}\right) \pi\left(\alpha_{x}(A)\right) E\left(\Delta_{2}\right) \Phi$ cannot vanish for all testfunctions $f$ with supp $\widetilde{f} \subset N$, since otherwise $E\left(\Delta_{1}\right) \pi(A) E\left(\Delta_{2}\right) \Phi=0$ for all $A \in \mathfrak{A}$. But this would imply $E\left(\Delta_{1}\right) F=0$ where $F \in \pi(\mathfrak{U})^{\prime}$ is the projection onto $\left[\pi(\mathfrak{U}) E\left(\Delta_{2}\right) \Phi\right]$ in contradiction to the assumption that $\pi$ is factorial.

4 If $C, C^{\prime} \subset \mathbb{R}^{4}$ and $\lambda \in \mathbb{R}$ we set $C \pm C^{\prime}=\left\{c \pm c^{\prime}: c \in C, c^{\prime} \in C^{\prime}\right\}$ and $\lambda \cdot C=\{\lambda c: c \in C\}$. 
In the second step one exploits locality by considering the commutator function $x \rightarrow K(x)=\left(\Phi, \pi\left(\left[\alpha_{x}\left(A_{1}\right), A_{2}\right]\right) \Phi\right)$, where $A_{1}, A_{2}$ are arbitrary local observables. So $K(x)$ vanishes in the causal complement of some bounded region $\mathcal{O} \subset \mathbb{R}^{4}$. If in addition the Fourier transform $\tilde{K}(p)$ vanishes on some open set $M \subset \mathbb{R}^{4}$, it follows from standard arguments $[20]$ that $\tilde{K}(p)$ is the discontinuity on the reals of some function which is analytic in $T_{+} \cup T_{-} \cup M$, where $T_{ \pm}=\left\{k \in \mathbb{C}^{4}: \operatorname{Im} k \in V_{ \pm}\right\}$. So $\tilde{K}(p)$ must also vanish at all real points of the envelope of holomorphy of $T_{+} \cup T_{-} \cup M$. Now for the case at hand, one deduces from the spectrum condition that $\tilde{K}(p)$ vanishes in some region $M=N \cap-N$, where $N$ is connected and contains the complement of $\left(\bar{V}_{+}-\operatorname{supp} \Phi\right)$ if $\operatorname{supp} \Phi$ is compact. For regions of this type the above envelope of holomorphy has been computed [20], and one finds that the boundary of $M$ is composed of hyperboloids $\left\{p:\left(p-p_{0}\right)^{2}=\mu_{0}^{2}\right\}$, where $p_{0} \in\left(\bar{V}_{+}-\right.$ $\operatorname{supp} \Phi) \cap-\left(\bar{V}_{+}-\operatorname{supp} \Phi\right)$ and $\mu_{0} \geqq 0$ are such that the hyperboloids do not intersect with the interior of $M$.

In the final step of the argument, which is of a purely geometric nature, one deduces from the above information the shape of the lower boundary of $\mathrm{sp} \mathscr{U}$ by different choices of $\operatorname{supp} \Phi$. QED.

In view of this result it seems plausible that also the full spectrum $\operatorname{sp} \mathscr{U}$ is a Lorentz-invariant set, but the above methods are not yet powerful enough to verify this conjecture.

Next, we want to exhibit continuity properties of the spectral projections $\Delta \rightarrow E(\Delta)$ of $P$ following from locality. To this end we study the subgroup of translations on a spacelike hyperplane of Minkowski space, i.e. $x \rightarrow U(x)$ with $x \cdot e=0$ for a fixed timelike vector $e \in V_{+}$. It will turn out that the joint spectrum of the corresponding generators is absolutely continuous, apart from a discrete point appearing in vacuum representations. Again, this result is known if the Lorentz transformations are implemented on $\mathscr{H}[14]$.

In the argument given below we shall deal with almost local operators transferring energy and momentum in a certain definite manner. Such operators were already used in [6] in a different context. In the present mathematical setting an operator $B \in \mathscr{B}(\mathscr{H})$ is said to be almost local if for some neighbourhood $\mathcal{O}$ of the origin in Minkowski space and any $\lambda>0$ one can find operators $B_{\lambda} \in \pi(\mathfrak{H}(\lambda \mathcal{O}))^{-}$ such that

$$
\lim _{\lambda \rightarrow \infty} \lambda^{i}\left\|B-B_{\lambda}\right\|=0 \quad \text { for all } i \in \mathbb{N}
$$

A concrete example of such an operator is

$$
B=\int d^{4} x f(x) \cdot \pi\left(\alpha_{x}(A)\right)
$$

where $f$ is a testfunction on $\mathbb{R}^{4}, A$ a local observable and the integral is defined as a weak integral. If the Fourier transform of $f$ in (2.7) has support $\Gamma \subset \mathbb{R}^{4}$ we say that $B$ transfers energy-momentum $\Gamma$.

Proposition 2.2. Let $(\pi, \mathscr{H})$ be a positive energy representation of $\mathfrak{A}$ and let $x \rightarrow U(x)$ be the subgroup of spacelike translations $x$ with $x \cdot e=0, e \in V_{+}$fixed. Then the joint spectrum of the corresponding generators $\mathbf{P}_{e}$ has the following structure: 
i) The non-atomic part of the spectrum of $\mathbf{P}_{e}$ is absolutely continuous with respect to Lebesgue measure. (ii) If there exists an atomic part in the spectrum of $\mathbf{P}_{e}$, it consists of the single point 0 , and the corresponding spectral subspace of $\mathscr{H}$ is spanned by vectors which are invariant under arbitrary translations $U(x)$. So $\pi$ is a vacuum representation.

Proof. Let $\mathscr{D}$ be the linear span of all vectors of the form $B \cdot \Psi$, where $\dot{\Psi} \in E\left(\Delta_{1}\right) \mathscr{H}$ and $B$ are almost local operators with energy-momentum transfer $\Gamma \subset \Delta_{2}$. It is obvious that $\mathscr{D} \subset E(\Delta) \mathscr{H}$, where $\Delta=\left(\Delta_{1} \cap \mathrm{sp} \mathscr{U}\right)+\Delta_{2}$. Moreover, if $\Delta_{1}$ and $\Delta_{2}$ are open sets and $\Delta_{1} \cap \operatorname{sp} \mathscr{U} \neq \emptyset$ then $\mathscr{D}$ is also dense in $E(\Delta) \mathscr{H}$. Otherwise, the Fourier transforms of $x \rightarrow E\left(\Delta_{1} \cap \operatorname{sp} \mathscr{U}\right) \cdot \pi\left(\alpha_{x}(A)\right) \Phi$ would vanish on $-\Delta_{2}$ for some non-zero vector $\Phi \in E(\Delta) \mathscr{H}$ and arbitrary local observables $A$; but this is only possible (cf. the proof of Proposition 2.1) if supp $\Phi \cap\left(\left(\Delta_{1} \cap \operatorname{sp} \mathscr{U}\right)+\Delta_{2}\right)=\emptyset$, which is clearly a contradiction.

Now given a $p \in \operatorname{sp} \mathscr{U}, p \neq 0$ one picks a $q \in \operatorname{sp} \mathscr{U}$ such that $q-(p-q)$ is a spacelike vector. (That such a $q$ exists can easily be seen if one exploits the fact that the lower boundary of sp $\mathscr{U}$ is a Lorentz-invariant set.) Since the set of spacelike vectors is open there exist also neighborhoods $\Delta_{p}$ of $p$ and $\Delta_{q}$ of $q$ such that the set $\Delta_{q}$ $\left(\Delta_{p}-\Delta_{q}\right)$ contains only spacelike vectors; it is therefore disjoint from sp $\mathscr{U}$. Then one considers the space $\mathscr{D}$, defined above, setting $\Delta_{1}=\Delta_{q}$ and $\Delta_{2}=\left(\Delta_{p}-\Delta_{q}\right)$. As was discussed, $\mathscr{D}$ is dense in $E(\Delta) \mathscr{H}$, where $\Delta=\left(\Delta_{q} \cap \operatorname{sp} \mathscr{U}\right)+\left(\Delta_{p}-\Delta_{q}\right)$ is again some neighborhood of $p$. The special choice of $\Delta_{q}$ makes it possible to show that for all $\Phi_{1}, \Phi_{2} \in \mathscr{D}$ the functions $x \rightarrow\left(\Phi_{1}, U(x) \Phi_{2}\right)$ are rapidly converging to 0 as $|x| \rightarrow \infty, x \cdot e=0$. In particular, they are absolutely integrable on the hyperplane $x \cdot e=0$, proving that the spectrum of the generators $\mathbf{P}_{e}$ is absolutely continuous on $E(\Delta) \mathscr{H}$. The proposition follows then, because $p \in \operatorname{sp} \mathscr{U}$ was arbitrary, apart from the condition $p \neq 0$.

It remains to establish the stated convergence properties of the above functions. Now for vectors of the form $B \cdot \Psi \in \mathscr{D}$, where $B$ transfers energy-momentum $\Gamma \subset\left(\Delta_{p}-\Delta_{q}\right)$ and $\Psi \in E\left(\Delta_{q}\right) \mathscr{H}$ one gets the estimate ${ }^{5}$

$$
\begin{aligned}
\left|\left(B_{1} \Psi_{1}, U(x) B_{2} \Psi_{2}\right)\right| & =\left|\left(\Psi_{1},\left[B_{1}^{*}, B_{2}(x)\right] U(x) \Psi_{2}\right)\right| \\
& \leqq\left\|\left[B_{1}^{*}, B_{2}(x)\right]\right\| \cdot\left\|\Psi_{1}\right\| \cdot\left\|\Psi_{2}\right\| .
\end{aligned}
$$

because $\left(\Delta_{q}-\left(\Delta_{p}-\Delta_{q}\right)\right) \cap \operatorname{sp} \mathscr{U}=\emptyset$ and therefore $B^{*} \cdot E\left(\Delta_{q}\right)=0$. Using the fact that the operators $B_{1}, B_{2}$ are almost local as well as the spacelike commutation properties of local observables, it is then easy to obtain the desired result

$$
\left|\left(B_{1} \Psi_{1}, U(x) B_{2} \Psi_{2}\right)\right| \leqq c_{i} \cdot(1+|x|)^{-i} \quad \text { for } x \cdot e=0,
$$

which can be extended to arbitrary vectors $\Phi_{1}, \Phi_{2} \in \mathscr{D}$ by taking finite sums. This bound holds for any $i \in \mathbb{N}$ and the coefficients $c_{i}$ depend continuously on the direction of $e$. QED.

It follows from this proposition that $E(\Delta)=0$ for any set $\Delta \subset \bar{V}_{+}$whose projection onto some spacelike hyperplane is a set of Lebesgue measure 0 (relative

5 We set $B(x)=U(x) B U(x)^{-1}$ for $B \in \mathscr{B}(\mathscr{H})$ 
to that plane). The fact that the estimates given in the proof hold uniformly for any compact set of vectors $e \in V_{+}$can be used to exhibit an even wider class of Lebesgue null-sets $\Delta$ for which $E(\Delta)=0$. However, for the present investigations we will only need the result that the joint spectrum of the momentum operators $\mathbf{P}$ is absolutely continuous in non-vacuum representations.

We will restrict our attention now to massive theories. There the most elementary systems are vacuum and single particle states which are separated from the multi-particle states in their sector by a mass gap. For later reference we give a formal characterization of the corresponding representations.

Definition. Let $(\pi, \mathscr{H})$ be a positive energy representation of some algebra of observable $\mathfrak{A}$. (i) $(\pi, \mathscr{H})$ is called a (massive) vacuum representation if $0 \in \operatorname{sp} \mathscr{U}$ and sp $\mathscr{U} \subset\{0\} \cup\left\{p: p^{2} \geqq \mu^{2}, p_{0}>0\right\}$ for some $\mu>0$. ii) $(\pi, \mathscr{H})$ is called a (massive) single particle representation, if the set $H_{m}=\left\{p: p^{2}=m^{2}, p_{0}>0\right\}$ is contained in the singular spectrum of $P$, and if $\operatorname{sp} \mathscr{U} \subset H_{m} \cup\left\{p: p^{2} \geqq M^{2}, p_{0}>0\right\}$ for some $M>m>0$.

According to Proposition 2.1 any positive energy representation where a part of the lower boundary of $\operatorname{sp} \mathscr{U}$ is isolated from the rest of the spectrum is either a vacuum or a single particle representation.

\section{Localization of Single Particle States}

We turn now to the analysis of the localization properties of states in massive single particle representations of $\mathfrak{U}$. As was explained in the Introduction, we shall find that these states can be interpreted as excitations of a specific vacuum state. Similar results were already obtained in [6] in a different mathematical setting. Our improvements on that analysis are twofold: first, Lorentz-covariance is no longer needed in the proofs, and second, we could verify that single particle representations and the corresponding vacuum representations are equivalent on the algebras $\mathfrak{U}^{c}(\mathscr{S})$. The latter result provides the basis for our analysis of multiparticle states.

For the subsequent discussion it is convenient to introduce the following notation: all points of the energy-momentum spectrum lying on the lower boundary $H_{m}=\left\{p: p^{2}=m^{2}, p_{0}>0\right\}$ of $\mathrm{sp} \mathscr{U}$ are marked by a tilde, $p^{\sim}=\left(\left(\mathbf{p}^{2}+\right.\right.$ $\left.\left.m^{2}\right)^{1 / 2}, \mathbf{p}\right)$. The symbol $\Delta^{\sim}$ stands for open, bounded subsets of $\mathbb{R}^{4}$ having only points $p^{\sim}$ in common with $\operatorname{sp} \mathscr{U}$, i.e. $\Delta^{\sim} \cap \operatorname{sp} \mathscr{U} \subset H_{m}$. Such regions exist because of the mass gap in $\operatorname{sp} \mathscr{U}$ in massive single particle representations.

We write $g(P)$ for the operators $\int g(p) d E(p)$, where $p \rightarrow g(p)$ are smooth functions on $\mathbb{R}^{4}$ (i.e. the derivatives of $g$ exist up to any order). If the function $g$ is not bounded, it is understood that $g(P)$ acts on its natural domain of definition. With this notation we can now state the basic theorem which expresses the fact that well localized states exist in massive single particle representations.

Theorem 3.1. Let $\Delta_{1}, \Delta_{2}$ be arbitrary neighbourhoods of a given pair of points $\tilde{p_{1}}$ and $\tilde{p_{2}}$. Then there exist regions $\tilde{\Delta}_{1}^{\tilde{C}} \subset \Delta_{1}, \Delta_{2}^{\sim} \subset \Delta_{2}$ and a family of almost local operators $B_{g}$ which are assigned to the smooth functions $g$ on $\mathbb{R}^{4}$, such that the following relations hold: 

i) $B \cdot E\left(\tilde{\Delta_{1}}\right) \neq 0$,
ii) $\left(g(P) \cdot B-B_{g}\right) \cdot E\left(\Delta_{1}^{\sim}\right)=0$,
iii) $E\left(\Delta_{2}^{\sim}\right) \cdot\left(g(P) \cdot B-B_{g}\right)=0$,
iv) $B \cdot E\left(\Delta_{1}^{\sim}\right)=E\left(\Delta_{2}^{\sim}\right) \cdot B \cdot E\left(\Delta_{1}^{\sim}\right)$.

Here $B$ denotes the operator attached to the constant function $e(p)=1$.

Let us comment first on the physical significance of this statement. The relevant information is contained in relation (ii), which says that the effect of the operation $g(P) \cdot B$ on any ensemble of particles with energy-momentum in $\Delta_{1}^{\tilde{1}}$ is well localized in configuration space. This holds, in spite of the fact that $g(P)$ is completely dislocalized, because $B$ filters out of these ensembles with overwhelming probability particles which enter some finite space-time region (cf. the heuristic remarks in the Introduction). So the energy-momentum content (the matter) of the resulting ensembles is essentially concentrated in that region and the action of $g(P)$ becomes effective only there. Relations (iii) and (iv) subsume some information on the energy-momentum transfer of $B$ and are a consequence of (ii).

Since the proof of this theorem is very technical we give it in the Appendix and sketch here the basic idea. We will make use of the fact that because of the mass gap in $\operatorname{sp} \mathscr{U}$ there exist almost local operators $B$, which map all single particle states with energy and momentum about $\tilde{p}$, say, into single particle states, and which annihilate all states with energy and momentum about some $q^{\sim} \neq p^{\sim}$. In particular, there are almost local operators $B_{0}$ and $B_{1}$ such that the ranges of $B_{0} E\left(\Delta_{1}^{\sim}\right)$ and $B_{1} U(x) B_{0} E\left(\Delta_{1}^{\sim}\right), x \in \mathbb{R}^{4}$ are contained in the single particle space $\mathscr{H}^{(1)}$ and $B_{1} E\left(\Delta_{1}^{\sim}\right)=0$. Now formally one has for fixed $\mathbf{q}$ the relation

$$
\int d^{3} x e^{-i \mathbf{q} \mathbf{x}} B_{1}(\mathbf{x}) \cdot e^{-i t P_{0}} \uparrow \mathscr{H}^{(1)}=e^{-i t\left([\mathbf{P}+\mathbf{q}]^{2}+m^{2}\right)^{1 / 2}} \cdot \int d^{3} x e^{-i \mathbf{q} \mathbf{x}} B_{1}(\mathbf{x}) \uparrow \mathscr{H}^{(1)}
$$

because $\int d^{3} x e^{-i \mathbf{q x}} B_{1}(\mathbf{x})$ transfers the momentum $\mathbf{q}$ and $P_{0}=\left(\mathbf{P}^{2}+m^{2}\right)^{1 / 2}$ on $\mathscr{H}^{(1)}$. So taking into account that $B_{1} \cdot E\left(\Delta_{1}^{\sim}\right)=0$ one gets

$$
\begin{aligned}
\int d^{3} x e^{-i \mathbf{q} \mathbf{x}}\left[B_{1}(t, \mathbf{x}), B_{0}\right] \cdot E\left(\Delta_{1}^{\sim}\right)= & \exp \left\{i t\left(\left(\mathbf{P}^{2}+m^{2}\right)^{1 / 2}-\left([\mathbf{P}+\mathbf{q}]^{2}+m^{2}\right)^{1 / 2}\right)\right\} \\
& \cdot \int d^{3} x e^{-i \mathbf{q} \mathbf{x}}\left[B_{1}(\mathbf{x}), B_{0}\right] \cdot E\left(\Delta_{1}^{\sim}\right) .
\end{aligned}
$$

But $\int d^{3} x e^{-i \mathbf{q x}}\left[B_{1}(t, \mathbf{x}), B_{0}\right]$ is for any $t$ an almost local operator because of locality, and therefore relation (3.2) is a special case of the crucial relation (ii) of the theorem with a definite function $g$. It is shown in the Appendix, how this result extends to arbitrary functions if one replaces in the above reasoning the commutators by suitable multiple commutators.

The following two corollaries are simple consequences of the theorem.

Corollary 3.2. Let $\mathcal{O}$ be a fixed neighbourhood of the origin in $\mathbb{R}^{4}$. Then for any $C \in \pi(\mathfrak{U}(\lambda \odot))^{\prime}$ and $\lambda>0$

$$
\left\|E\left(\Delta_{1}^{\sim}\right) B^{*}[g(P), C] B E\left(\Delta_{1}^{\sim}\right)\right\| \leqq h(\lambda) \cdot\|C\|,
$$


where $B, g(P)$ and $E\left(\Delta_{1}^{\sim}\right)$ are the same as in the preceding theorem. The function $h$ does not depend on $C$ and is rapidly decreasing ${ }^{6}$.

Proof. According to relation (iv) of the above theorem, one has $E\left(\Delta_{1}^{\tilde{1}}\right) B^{*}=$ $E\left(\Delta_{1}^{\tilde{C}}\right) B^{*} E\left(\Delta_{2}^{\tilde{2}}\right)$, and using also (ii) and (iii) one gets

$$
\begin{aligned}
E\left(\Delta_{1}^{\tilde{1}}\right) B^{*}\left[B_{g}, C\right] E\left(\Delta_{1}^{\tilde{r}}\right) & =E\left(\Delta_{1}^{\tilde{\tau}}\right) B^{*}[g(P) B, C] E\left(\Delta_{1}^{\tilde{r}}\right) \\
& =E\left(\Delta_{1}^{\tilde{1}}\right) B^{*}[g(P), C] B E\left(\Delta_{1}^{\tilde{r}}\right)+E\left(\Delta_{1}^{\tilde{1}}\right) B^{*} g(P)[B, C] E\left(\Delta_{1}^{\tilde{r}}\right) .
\end{aligned}
$$

This implies

$$
\left\|E\left(\Delta_{1}^{\tilde{1}}\right) B^{*}[g(P), C] B E\left(\Delta_{1}^{\tilde{1}}\right)\right\| \leqq\|B\| \cdot\left\|\left[B_{g}, C\right]\right\|+\sup _{p \in \Delta_{\tilde{2}}^{\tilde{2}}}|g(p)| \cdot\|B\| \cdot\|[B, C]\|
$$

and bearing in mind that $B$ and $B_{g}$ are almost local operators one arrives at the conclusion. QED.

The algebras $\pi(\mathfrak{A}(\mathcal{O}))^{\prime}$ in the corollary contain the observables in the causal complement of $\mathcal{O}$ because of locality, $\pi(\mathfrak{A}(\mathcal{O}))^{\prime} \supset \pi\left(\mathfrak{A}\left(\mathcal{O}^{\prime}\right)\right)^{-}$. Only if $\pi$ describes a particle with ordinary Bose or Fermi statistics both algebras coincide, i.e. duality holds [3]. It is essential for the direct construction of the charge conjugate sectors in the parastatistics case that the corollary holds for the in general larger algebras $\pi(\mathfrak{H}(\mathcal{O}))^{\prime}[17]$.

Corollary 3.3. There exists a vector in $\mathscr{H}$ inducing a state $\omega$ such that

$$
\left|\partial_{\mu} \omega\left(\alpha_{x}(A)\right)\right| \leqq h\left(|\mathbf{x}|-\left|x_{0}\right|\right) \cdot\|A\| \quad \text { for } A \in \mathfrak{U}(\mathcal{O})
$$

where $\mathcal{O}$ is any fixed bounded region, $\partial_{\mu}, \mu=0,1,2,3$ are the derivatives with respect to the coordinates of $x$ and $h$ is a function which does not depend on $A$ and is rapidly decreasing.

Proof. If $\left(|\mathbf{x}|-\left|x_{0}\right|\right)$ is sufficiently large, the region $\mathcal{O}+x$ is contained in the causal complement of a double cone with diameter $\left(|\mathbf{x}|-\left|x_{0}\right|\right)$, which is centered at the origin of $\mathbb{R}^{4}$. Setting $g(p)=p_{\mu}$ in the preceding corollary, where $p_{\mu}, \mu=$ $0,1,2,3$ are the coordinates of $p$, it follows that

$$
\left\|E\left(\Delta_{1}^{\tilde{1}}\right) B^{*}\left[P_{\mu}, \pi\left(\alpha_{x}(A)\right)\right] B E\left(\Delta_{1}^{\tilde{1}}\right)\right\| \leqq h\left(|\mathbf{x}|-\left|x_{0}\right|\right) \cdot\|A\| .
$$

Thus any normalized vector in the range of $B \cdot E\left(\Delta_{1}^{\tilde{1}}\right)$ induces a state $\omega$ with the desired property. QED.

We can state now the result which relates any massive single particle representation $(\pi, \mathscr{H})$ to a particular vacuum representation $\left(\pi_{0}, \mathscr{H}_{0}\right)$ of $\mathfrak{A}$. In this statement we deal with sequences of vectors $x$ tending to spacelike infinity, which means that $\left(|\mathbf{x}|-\left|x_{0}\right|\right) \rightarrow \infty$.

Theorem 3.4. There exists an irreducible, massive vacuum representation $\left(\pi_{0}, \mathscr{H}_{0}\right)$

6 A function $h$ is said to be rapidly decreasing if $\lim _{\lambda \rightarrow \infty} \lambda^{i} h(\lambda)=0$ for all $i \in \mathbb{N}$. We shall reserve the symbol $h$ for such functions 
of $\mathfrak{U}$ such that for any $A \in \mathfrak{A}$ and any sequence of vectors $x$ tending to spacelike infinity

$$
w-\lim _{x} \pi\left(\alpha_{x}(A)\right)=\omega_{0}(A) \cdot 1
$$

where $\omega_{0}$ is the state induced by the (up to a phase unique) vector in $\mathscr{H}_{0}$ representing the vacuum.

Proof. Let $A \in \mathfrak{A}(\mathcal{O})$ be any local observable. Then, for $x$ tending to spacelike infinity, all weak limit points of $\pi\left(\alpha_{x}(A)\right)$ are multiples of the identity due to locality and the fact that $\pi$ is a factorial representation. In particular one has for any normal state $\omega$

$$
w-\lim _{x}\left[\pi\left(\alpha_{x}(A)\right)-\omega\left(\alpha_{x}(A)\right) \cdot 1\right]=0 .
$$

So in order to prove the convergence of $\pi\left(\alpha_{x}(A)\right)$ it suffices to establish the convergence of $\omega\left(\alpha_{x}(A)\right)$ for some vectorstate $\omega$ of the type exhibited in the preceding corollary. Now

$$
\omega\left(\alpha_{x}(A)\right)-\omega\left(\alpha_{y}(A)\right)=\int_{\mathscr{C}} d z^{\mu} \partial_{\mu} \omega\left(\alpha_{z}(A)\right)
$$

where $\mathscr{C}$ is any smooth curve connecting $x$ and $y$, and using the bounds on $\partial_{\mu} \omega\left(\alpha_{z}(A)\right)$ given in the preceding corollary one arrives at

$$
\left|\omega\left(\alpha_{x}(A)\right)-\omega\left(\alpha_{y}(A)\right)\right| \leqq \int_{\mathscr{C}} d l h\left(|\mathbf{z}|-\left|z_{0}\right|\right) \cdot\|A\|,
$$

where $d l$ denotes the infinitesimal line element. Taking into account that $h(\lambda)$ converges rapidly to 0 for $\lambda \rightarrow \infty$ and that the region $\left\{z:|\mathbf{z}|-\left|z_{0}\right|>R\right\}$ is connected, it is easy to exhibit curves $\mathscr{C}$ giving the bound

$$
\mid \omega\left(\alpha_{x}(A)-\omega\left(\alpha_{y}(A)\right) \mid \leqq\left(\int_{|\mathbf{x}|-\left|x_{0}\right|}^{\infty} d \lambda h(\lambda)+\int_{|\mathbf{y}|-\left|y_{0}\right|}^{\infty} d \lambda h(\lambda)\right) \cdot\|A\| .\right.
$$

Hence if $x$ tends to spacelike infinity, $\omega \alpha_{x}(\cdot)$ converges to some state $\omega_{0}$ on $\mathfrak{A}$ which is invariant under translations.

It remains to show that $\omega_{0}$ is a vectorstate in an irreducible'massive vacuum representation. Let $\left(\pi_{0}, \mathscr{H}_{0}\right)$ be the representation of $\mathfrak{U}$ obtained from $\omega_{0}$ by the GNS-construction, and let $\Omega$ be the corresponding cyclic vector. Since $\omega_{0} \alpha_{x}(\cdot)=$ $\omega_{0}(\cdot)$, there exists a unitary representation of the translations on $\mathscr{H}_{0}$ defined by

$$
U_{0}(x) \cdot \pi_{0}(A) \Omega=\pi_{0}\left(\alpha_{x}(A)\right) \Omega, \quad A \in \mathfrak{U} .
$$

Moreover, if $A_{1}, A_{2}$ are local, the above estimate implies that the continuous functions $x \rightarrow \omega \alpha_{y}\left(A_{1} \alpha_{x}\left(A_{2}\right)\right)$ converge uniformly on compact regions to $x \rightarrow$ $\omega_{0}\left(A_{1} \alpha_{x}\left(A_{2}\right)\right)$ as $y$ tends to spacelike infinity, and therefore this function is also continuous. This shows that $x \rightarrow U_{0}(x)$ is continuous. By the same token one finds that

$$
\mathrm{w}-\lim _{y} \int d^{4} x f(x) \pi\left(\alpha_{y}\left(A_{1} \alpha_{x}\left(A_{2}\right)\right)\right)=\int d^{4} x f(x) \omega_{0}\left(A_{1} \alpha_{x}\left(A_{2}\right)\right) \cdot 1
$$


for any testfunction $f$, and from this one gets by standard arguments [10] that $\operatorname{sp} \mathscr{U}_{0}+\operatorname{sp} \mathscr{U} \subset \operatorname{sp} \mathscr{U}$. Hence

$$
\operatorname{sp} \mathscr{U}_{0} \subset\{0\} \cup\left\{p: p^{2} \geqq \mu^{2}, p_{0}>0\right\},
$$

where $\mu=M-m$ is the upper mass gap in sp $\mathscr{U}$. Also, $0 \in \operatorname{sp} \mathscr{U}_{0}$ because $\Omega$ is invariant under $U_{0}(x)$.

For the proof that $\left(\pi_{0}, \mathscr{H}_{0}\right)$ is irreducible it suffices to show that 0 is a simple eigenvalue of the generators of $U_{0}(x)$. From Corollary 3.2 it follows that for $A \in \mathfrak{A}(\mathcal{O})$

$$
\left\|E\left(\Delta_{1}^{\tilde{r}}\right) B^{*}\left(\pi\left(\alpha_{y}(A)\right)-\omega_{0}(A) \cdot 1\right) B E\left(\Delta_{1}^{\tilde{r}}\right)\right\| \leqq \int_{|\mathbf{y}|-\left|y_{0}\right|}^{\infty} d \lambda h(\lambda) \cdot\|A\|,
$$

so this expression converges to 0 if $y$ tends to spacelike infinity. On the other hand one gets for any $\Psi \in \mathscr{H}$ with supp $\Psi \subset \Delta_{1}^{\tilde{1}}$ and $B \Psi \neq 0$

$$
\begin{aligned}
\lim _{y} \int d^{4} x f(x)\left(B \Psi, \pi\left(\alpha_{y}\left(A_{1}\right) \alpha_{x+y}\left(A_{2}\right)\right) B \Psi\right) \\
=\lim _{y}\left\{\int d^{4} x f(x)\left(B \Psi, \pi\left(\alpha_{y}\left(A_{1}\right)\right) B E\left(\Delta_{1}^{\tilde{1}}\right) \pi\left(\alpha_{x+y}\left(A_{2}\right)\right) \Psi\right)\right. \\
\left.+\int d^{4} x f(x)\left(B \Psi, \pi\left(\alpha_{y}\left(A_{1}\right)\right) B\left[1-E\left(\Delta_{1}^{\sim}\right)\right] \pi\left(\alpha_{x+y}\left(A_{2}\right)\right) \Psi\right)\right\},
\end{aligned}
$$

and the last term vanishes if in momentum space $f$ has its support in some sufficiently small neighbourhood of the origin. To the remaining term one can apply the above estimate giving

$$
\int d^{4} x f(x)\left(\omega_{0}\left(A_{1} \alpha_{x}\left(A_{2}\right)\right)-\omega_{0}\left(A_{1}\right) \omega_{0}\left(A_{2}\right)\right)=0
$$

for all $A_{1}, A_{2} \in \mathfrak{U}$ and the restricted testfunctions $f$. Hence 0 is a non-degenerate eigenvalue of the generators of $U_{0}(x)$, and this completes the proof of the theorem.

QED.

It follows also from the above argument that the vacuum representation $\pi_{0}$ is normal relative to $\pi$ on any local algebra $\mathfrak{A}(\mathcal{O})$. In our next theorem we will strengthen this statement. For this purpose we need some notation: let $\mathcal{O}$ be any open double cone whose closure $\overline{\mathcal{O}}$ lies in the spacelike complement of the origin in $\mathbb{R}^{4}$, and let $a$ be any point. Then the region $\mathscr{S}=a+\bigcup_{\lambda>0} \lambda \cdot \mathcal{O}$ is called a spacelike cone with apex $a$. Clearly, $\mathscr{S}-a$ is an open, convex cone, and $(2 a-\mathscr{S}) \subset \mathscr{S}^{\prime}$. Moreover, $\mathfrak{U}(\mathcal{O}) \subset \mathfrak{U}^{c}(\mathscr{S})$ for any $\mathcal{O} \subset \mathscr{S}^{\prime}$ because of locality.

Theorem 3.5. Let $(\pi, \mathscr{H})$ be a massive single particle representation of $\mathfrak{A}$ with a cyclic vector, and let $\left(\pi_{0}, \mathscr{H}_{0}\right)$ be the associated vacuum representation. Then the restrictions of $\pi$ and $\pi_{0}$ to the algebras $\mathfrak{A}^{c}(\mathscr{S})$ are unitarily equivalent, i.e. for any spacelike cone $\mathscr{S}$ there exists an isometry $V$ of $\mathscr{H}$ onto $\mathscr{H}_{0}$ such that

$$
V \pi(A)=\pi_{0}(A) V \quad \text { for } A \in \mathfrak{A}^{c}(\mathscr{S}) .
$$

Proof. Since the translations $\alpha_{x}$ are unitarily implemented in the representations $\pi$ and $\pi_{0}$, it suffices to consider spacelike cones $\mathscr{S}$ containing some neighbourhood $\mathcal{O}$ of the origin in Minkowski space. Then, if $a$ is the apex of $\mathscr{S}$, one has $[\lambda+1] \mathcal{O} \subset$ $\mathscr{S}+\lambda a$ for $\lambda>0$ and consequently

$$
\pi\left(\mathfrak{A}^{c}(\mathscr{S}+\lambda a)\right) \subset \pi(\mathfrak{H}([\lambda+1] \mathcal{O}))^{\prime} .
$$


It follows therefore from Corollary 3.2 (using the same notation) that for any $A \in \mathfrak{U}^{c}(\mathscr{S})$

$$
\left\|\frac{d}{d \lambda} E\left(\Delta_{1}^{\tilde{1}}\right) B^{*} \pi\left(\alpha_{\lambda a}(A)\right) B E\left(\Delta_{1}^{\sim}\right)\right\| \leqq h(\lambda+1) \cdot\|A\|,
$$

and $h(\lambda+1)$ converges rapidly to 0 for $\lambda \rightarrow \infty$. Now $\lambda a$ tends to spacelike infinity for $\lambda \rightarrow \infty$ because (with our choice of $\mathscr{S}$ ) $a$ is a spacelike vector, and therefore

$$
\left\|E\left(\Delta_{1}^{\sim}\right) B^{*} \pi\left(\alpha_{\lambda a}(A)-\omega_{0}(A) \cdot 1\right) B E\left(\Delta_{1}^{\sim}\right)\right\| \leqq \int_{\lambda}^{\infty} d \lambda^{\prime} h\left(\lambda^{\prime}+1\right) \cdot\|A\| .
$$

This shows that the restriction of $\omega_{0}$ to $\mathfrak{A}^{c}(\mathscr{S})$ can be uniformly approximated by vectorstates in the representation $\pi$, hence $\omega_{0}(A)=\bar{\omega}_{0}(\pi(A))$ for $A \in \mathfrak{A}^{c}(\mathscr{S})$, where $\bar{\omega}_{0}$ is some normal state on $\pi\left(\mathfrak{Q}^{c}(\mathscr{S})\right)^{-}$.

If $\bar{\omega}_{0}$ is induced by a vector $\bar{\Omega} \in \mathscr{H}$ which is cyclic for $\pi\left(\mathfrak{U}^{c}(\mathscr{S})\right)^{-}$one can define an isometry $V$ by

$$
V \cdot \pi(A) \bar{\Omega}=\pi_{0}(A) \Omega \quad \text { for } A \in \mathfrak{A}^{c}(\mathscr{S}) .
$$

Clearly, $V$ maps $\mathscr{H}$ onto $\mathscr{H}_{0}$ because the vacuum vector $\Omega$ is cyclic for $\pi_{0}\left(\mathfrak{H}^{c}(\mathscr{S})\right)$ according to the Reeh-Schlieder theorem ${ }^{7}[21]$. Also, $V \pi(A)=\pi_{0}(A) V$ for $A \in$ $\mathfrak{A}^{c}(\mathscr{S})$, so the proof of the theorem is complete if one can establish the existence of $\bar{\Omega}$. Now let $\Phi \in \mathscr{H}$ be any vector which is analytic for the energy [20]. It follows then by a slight generalization of the Reeh-Schlieder theorem that

$$
\left[\pi\left(\mathfrak{H}^{c}(\mathscr{S})\right)^{\prime} \Phi\right] \supset\left[\pi(\mathfrak{U})^{\prime} \pi(\mathfrak{U}(\mathscr{S}))^{-} \Phi\right]=\left[\pi(\mathfrak{U})^{\prime} \pi(\mathfrak{U})^{-} \Phi\right]=\mathscr{H},
$$

where the last equality sign holds because $\pi$ is a factorial representation. Hence $\Phi$ is separating for $\pi\left(\mathfrak{H}^{c}(\mathscr{S})\right)^{-}$, and therefore any normal state on $\pi\left(\mathfrak{U}^{c}(\mathscr{S})\right)^{-}$ is induced by some vector in $\mathscr{H}_{-}[9 ; \mathrm{Thm} .2 .7 .9]$; in particular there exists a vector $\overline{\bar{\Omega}} \in \mathscr{H}$ such that $\bar{\omega}_{0}(C)=(\overline{\bar{\Omega}}, C \overline{\bar{\Omega}})$ for $C \in \pi\left(\mathfrak{H}^{c}(\mathscr{S})\right)^{-}$. Moreover, $\overline{\bar{\Omega}}$ is also separating for $\pi\left(\mathfrak{Q}^{c}(\mathscr{S})\right)^{-}$which may be seen as follows: if $C \overline{\bar{\Omega}}=0$ for $C \in \pi\left(\mathfrak{A}^{c}(\mathscr{S})\right)^{-}$, one has also

$$
\|C U(x) \overline{\bar{\Omega}}\|^{2}=\bar{\omega}_{0}\left(\left(C^{*} C\right)(-x)\right)=\bar{\omega}_{0}\left(C^{*} C\right)=\|C \overline{\bar{\Omega}}\|^{2}=0
$$

for $x \in \mathscr{S}-a$, and using the spectrum condition for $U(x)$ one concludes that $C U(x) \overline{\bar{\Omega}}=0$ for all $x$. So if one integrates $x \rightarrow U(x) \overline{\bar{\Omega}}$ with a suitable testfunction, one can construct a vector which is analytic for the energy and which is annihilated by $C$. As was shown, this is only possible if $C=0$. Thus $\overline{\bar{\Omega}}$ is separating for $\pi\left(\mathfrak{U}^{c}(\mathscr{S})\right)^{-}$, and according to the theorem quoted above there exists then a vector $\bar{\Omega} \in \mathscr{H}$ inducing $\bar{\omega}_{0}$, and

$$
\left[\pi\left(\mathfrak{U}^{c}(\mathscr{S})\right)^{-} \bar{\Omega}\right]=\left[\pi\left(\mathfrak{U}^{c}(\mathscr{S})\right)^{-} \Phi\right]=\left[\pi(\mathfrak{U})^{-} \Phi\right],
$$

where the second equality sign follows again from the Reeh-Schlieder theorem.

Finally, if there exists any cyclic vector $\Psi \in \mathscr{H}$ for $\pi(\mathfrak{A})$, then there exists also

7 Weak additivity is not needed here since $\mathfrak{A}(x-\mathscr{S}) \subset \mathfrak{A}^{c}(\mathscr{S})$ for $x \in 3 a-\mathscr{S}$ and $\bigcup_{y} \mathfrak{A}(y-\mathscr{S})$ is norm-dense in $\mathfrak{U}$ 
a vector $\Phi \in \mathscr{H}$ which is cyclic and analytic for the energy, because $U(x) \in \pi(\mathfrak{U})^{-}$. (A simple example is $\Phi=\exp \left(-P_{0}\right) \Psi$.) So one may set in the above relation $\left[\pi(\mathfrak{U})^{-} \Phi\right]=\mathscr{H}$, and this completes the proof of the theorem. QED.

If in this theorem one drops the assumption that $\mathscr{H}$ contains a cyclic vector, then it still follows from the above argument that the restrictions of $\pi$ and $\pi_{0}$ to $\mathfrak{A}^{c}(\mathscr{S})$ are quasi-equivalent. This result cannot be improved without further information, because the representations $\pi$ and $\pi_{0}$ may live on Hilbert spaces whose dimensions are of different cardinality. However, if $\mathscr{H}$ is separable then there always exists a cyclic vector in $\mathscr{H}$. For the proof of this assertion we remark that $\pi_{0}\left(\mathfrak{H}^{c}(\mathscr{S})\right)^{-}$, and consequently $\pi\left(\mathfrak{U}^{c}(\mathscr{S})\right)^{-}$is a von Neumann algebra of type III according to an argument of Driessler [22]. But on a separable Hilbert space any von Neumann algebra of type III containing the identity has a cyclic vector [9], and any vector which is cyclic for $\pi\left(\mathfrak{U}^{c}(\mathscr{S})\right)^{-}$is a fortiori cyclic for $\pi(\mathfrak{U})^{-}$.

In conclusion we remark that the analysis of this section can be carried out in any number of space-time dimensions. Only in the model-world of two dimensions Theorems 3.4 and 3.5 have to be modified, because there it is possible that the states $\omega \alpha_{x}(\cdot)$ converge to different vacua if $x$ tends to spacelike infinity in the right or left wedge, respectively. (As is well known, this does happen in the so-called soliton sectors of some two dimensional models; see e.g. [23].) Since it is obvious how to modify the statements in this case we omit the details.

\section{Composition of Sectors}

If $\pi_{1}$ and $\pi_{2}$ are representations of $\mathfrak{A}$ describing single particle states there should also exist a representation describing states where both particles are present. At this point the localization properties of particles are important, because they lead to a natural definition of such composed representations.

For representations satisfying the $\mathrm{DHR}^{8}$-selection criterion (1.6), a composition can be defined because the equivalence of $\pi$ and $\pi_{0}$ on the algebras $\mathfrak{A}\left(\mathcal{O}^{\prime}\right)$ implies that there are localized morphisms $\rho$ of $\mathfrak{A}$ such that $\pi_{0}{ }^{\circ} \rho \simeq \pi$. Hence if $\pi_{0}{ }^{\circ} \rho_{1} \simeq \pi_{1}$ and $\pi_{0}{ }^{\circ} \rho_{2} \simeq \pi_{2}$ there exists also the composed representation $\pi_{0}{ }^{\circ} \rho_{1} \rho_{2}$, and this definition of a composition is natural because the equivalence class (the sector) of the composed representation depends only on $\pi_{1}$ and $\pi_{2}$, but not on the particular choice of the morphisms $\rho_{1}$ and $\rho_{2}$ [DHR I].

We shall see that a similar result holds also for representations which are localizable in spacelike cones $\mathscr{S}$.

Definition. A representation $(\pi, \mathscr{H})$ of $\mathfrak{U}$ is said to be localizable in cones relative to a vacuum representation $\left(\pi_{0}, \mathscr{H}_{0}\right)$, if for any spacelike cone $\mathscr{S}$ there exists an isometry $V$ from $\mathscr{H}$ onto $\mathscr{H}_{0}$ such that

$$
V \pi(A)=\pi_{0}(A) V \text { for } A \in \mathfrak{A}^{c}(\mathscr{S}) .
$$

We denote the class of representations which are localizable in cones relative to

8 In the following we will refer to the articles [3] and [4] as [DHR I] and [DHR II], respectively 
a fixed vacuum representation $\pi_{0}$ by $(S)$. If $\mathfrak{A}$ has more than one vacuum representation there exist several such classes, but it is not necessary to distinguish them here. As we have shown in Theorem 3.5, all massive single particle representations are elements of some $(S)$.

Using the freedom to choose the location of the cone $\mathscr{S}$ in relation (4.1) it follows that $\|\pi(A)\|=\left\|\pi_{0}(A)\right\|$ for all $A \in \mathfrak{A}$. So any $\pi \in(S)$ can be regarded as a representation of $\pi_{0}(\mathfrak{Q})$, and identifying the algebras $\pi_{0}(\mathfrak{H})$ and $\mathfrak{A}$ we may drop in the following the symbol $\pi_{0}$. It will also be convenient to fix $\mathscr{H}_{0}$ as representation space for the representations in $(S)$. In particular, if $\pi \in(S)$ we may choose any spacelike cone $\mathscr{S}_{0}$ and use the corresponding isometry $V$ from relation (4.1) for the definition of a representation $\rho$ on $\mathscr{H}_{0}$ which is equivalent to $\pi$,

$$
\rho(A)=V \pi(A) V^{-1}, \quad A \in \mathfrak{A} .
$$

This representation acts trivially on $\mathfrak{A}^{c}\left(\mathscr{S}_{0}\right)$

$$
\rho(A)=A, \quad A \in \mathfrak{Q}^{c}\left(\mathscr{S}_{0}\right),
$$

and we refer to this important fact by saying that $\rho$ is localized in $\mathscr{S}_{0}$.

If one performs this construction for any other spacelike cone $\mathscr{S}_{1}$, one obtains a representation $\rho_{1}$ on $\mathscr{H}_{0}$ which is equivalent to $\rho$, so there exists a unitary operator $V \in \mathscr{B}\left(\mathscr{H}_{0}\right)$ such that

$$
V \rho(A)=\rho_{1}(A) V, \quad A \in \mathfrak{A} .
$$

The localization properties of $\rho$ and $\rho_{1}$ imply that

$$
V A=A V
$$

for $A \in \mathfrak{H}^{c}\left(\mathscr{S}_{0}\right) \cap \mathfrak{A}^{c}\left(\mathscr{S}_{1}\right)$, hence if $\mathscr{S}_{2}$ is a spacelike cone containing $\mathscr{S}_{0}$ and $\mathscr{S}_{1}$, it follows from the duality assumption that

$$
V \in \mathfrak{A}\left(\mathscr{S}_{2}\right)^{-} \text {. }
$$

So the intertwiners $V$ are related to the algebra $\mathfrak{A}$, but in contrast to the case considered by DHR they need not be elements of $\mathfrak{U}$. Also, $\rho(\mathfrak{H})$ may not be contained in $\mathfrak{U}$, so a composition of the representations $\rho$ is a priori not defined.

This problem is solved by extending $\rho$ to some larger algebra on which it acts as a morphism. Here one makes use of the fact that the representations $\rho$ are weakly continuous on the algebras $\mathfrak{I}^{c}(\mathscr{S})$, so they can be extended to the weak closures $\mathfrak{A}^{c}(\mathscr{S})^{-}$by continuity. Since the set of regions $\mathscr{S}$ is not directed, it is not clear whether there exists an extension of $\rho$ to the $C^{*}$-algebra which is generated by all the algebras $\mathfrak{A}^{c}(\mathscr{S})^{-}$, where $\mathscr{S}$ are arbitrary spacelike cones. But given some auxiliary spacelike cone $\mathscr{S}_{a}$, the family of algebras $\mathfrak{Q}^{c}\left(\mathscr{S}_{a}+x\right)^{-}, x \in \mathbb{R}^{4}$ is an increasing net with respect to the partial ordering

$$
x \leqq y \Leftrightarrow \mathscr{S}_{a}+x \supset \mathscr{S}_{a}+y,
$$

and using relation (4.4) one can define an extension of $\rho$ to the algebra ${ }^{9}$

$$
\mathfrak{B}^{\mathscr{S}_{a}}=\bigcup_{x}^{C^{*}} \mathfrak{I}^{c}\left(\mathscr{S}_{a}+x\right)^{-} .
$$

9 Because of duality the algebras $\mathfrak{H}^{c}(\mathscr{S})^{-}$and $\mathfrak{H}(\mathscr{S})^{\prime}$ coincide. But we distinguish them in order to display at which points of our argument duality is used 
Clearly $\mathfrak{B}^{\mathscr{S}_{a}}$ depends only on the class $\left\{\mathscr{S}_{a}+x, x \in \mathbb{R}^{4}\right\}$, so $\mathfrak{B}^{\mathscr{S}_{a}}=\mathfrak{B}^{\mathscr{S}_{a}+x}$ for any $x$.

Lemma 4.1. Let $\rho \in(S)$ be a representation of $\mathfrak{U}$ on $\mathscr{H}_{0}$ which is localized in $\mathscr{S}_{0}$. Then there exists a unique morphism $\rho^{\mathscr{S}_{a}}$ from $\mathfrak{B}^{\mathscr{S}_{a}}$ into $\mathscr{B}\left(\mathscr{H}_{0}\right)$ which is weakly continuous on $\mathfrak{U}^{c}\left(\mathscr{S}_{a}+x\right)^{-}$for any $x$ and coincides with $\rho$ on $\mathfrak{A}$. If $\mathscr{S}_{0}$ is spacelike separated from $\mathscr{S}_{a}+x$ for some $x, \mathfrak{B}^{\mathscr{S}_{a}}$ is mapped into itself by $\rho^{\mathscr{S}_{a}}$.

Proof. Let $A \in \mathfrak{A}^{c}\left(\mathscr{S}_{a}+x\right)$ and let $\rho_{1}$ be equivalent to $\rho$ and localized in $\mathscr{S}_{a}+x$. Then, if $V$ is a unitary intertwiner from $\rho_{1}$ to $\rho$, we get from (4.4)

$$
\rho(A)=V A V^{-1} \text {. }
$$

Thus $\rho$ is weakly continuous on $\mathfrak{H}^{c}\left(\mathscr{S}_{a}+x\right)$, and setting

$$
\rho^{\mathscr{S}_{a}}(B)=V B V^{-1}
$$

for $B \in \mathfrak{U}^{c}\left(\mathscr{S}_{a}+x\right)^{-}$, we get the unique weakly continuous extension of $\rho$ to $\mathfrak{U}^{c}\left(\mathscr{S}_{a}+x\right)^{-}$. Since $\mathfrak{B}^{\mathscr{S}_{a}}$ is the $\mathrm{C}^{*}$-inductive limit of the net of algebras $\left(\mathfrak{U}^{c}\left(\mathscr{S}_{a}+x\right)^{-}\right)_{x \in \mathbb{R}^{4}}$ this relation determines $\rho^{\mathscr{S}_{a}}$ on all of $\mathfrak{B}^{\mathscr{S}_{a}}$.

If $\mathscr{S}_{0}$ is spacelike separated from $\mathscr{S}_{a}+x$ for some $x$, then the algebras $\mathfrak{U}^{c}\left(\mathscr{S}_{a}+y\right)^{-}$, where $y$ is such that $\mathscr{S}_{a}+y \subset \mathscr{S}_{0}^{\prime}$, already generate $\mathfrak{B}^{\mathscr{S}_{a}}$. Now

$$
\rho^{\mathscr{S}_{a}}\left(\mathfrak{H}^{c}\left(\mathscr{S}_{a}+y\right)^{-}\right)=\rho\left(\mathfrak{U}^{c}\left(\mathscr{S}_{a}+y\right)\right)^{-}
$$

because of the weak continuity of $\rho^{\mathscr{S}_{a}}$ on $\mathfrak{A}^{c}\left(\mathscr{S}_{a}+y\right)^{-}$. Moreover, because of locality and the fact that $\rho$ is a morphism, we have

$$
\rho\left(\mathfrak{U}^{c}\left(\mathscr{S}_{a}+y\right)\right)^{-} \subset \rho\left(\mathfrak{U}\left(\mathscr{S}_{a}+y\right)\right)^{\prime} .
$$

But $\rho$ is localized in $\mathscr{S}_{0} \subset\left(\mathscr{S}_{a}+y\right)^{\prime}$, so it acts trivially on $\mathfrak{A}\left(\mathscr{S}_{a}+y\right)$. Therefore we get from the above relations and duality

$$
\rho^{\mathscr{S}_{a}}\left(\mathfrak{U}^{c}\left(\mathscr{S}_{a}+y\right)^{-}\right) \subset \mathfrak{U}\left(\mathscr{S}_{a}+y\right)^{\prime}=\mathfrak{U}^{c}\left(\mathscr{S}_{a}+y\right)^{-},
$$

which gives the desired result $\rho^{\mathscr{S}_{a}}\left(\mathfrak{B}^{\mathscr{S}_{a}}\right) \subset \mathfrak{B}^{\mathscr{S}_{a}}$. QED.

We mention that $\rho^{\mathscr{S}_{a}}=\rho^{\mathscr{S}_{a}+x}$ for any $x$, and we will use this fact occasionally. The set of representations $\rho \in(S)$ on $\mathscr{H}_{0}$ which are localized in a fixed spacelike cone $\mathscr{S}_{0}$ will be denoted by $\Delta_{\mathscr{S}_{0}}$. Using the extensions $\rho^{\mathscr{S}_{a}}$ constructed in the previous lemma it is possible to define a product in $\Delta_{\mathscr{S}_{0}}$.

Definition. $\rho_{1} \rho_{2}:=\rho_{1}^{\mathscr{S}_{a} \circ} \rho_{2}$ for $\rho_{1}, \rho_{2} \in \Delta_{\mathscr{S}_{0}}, \mathscr{S}_{a} \subset \mathscr{S}_{0}^{\prime}$.

This definition is reasonable in view of the following theorem.

Theorem 4.2. i) $\rho_{1} \rho_{2} \in \dot{\Delta}_{\mathscr{S}_{0}}$, ii) $\rho_{1} \rho_{2}$ does not depend on the choice of the auxiliary cone $\mathscr{S}_{a} \subset \mathscr{S}_{0}^{\prime}$. iii) If $\hat{\rho}_{i} \simeq \rho_{i}, i=1,2$ and $\hat{\rho}_{1}, \hat{\rho}_{2} \in \Delta_{\hat{\mathscr{S}}_{0}}$ for some spacelike cone $\hat{\mathscr{S}}_{0}$, then $\rho_{1} \rho_{2} \simeq \hat{\rho}_{1} \hat{\rho}_{2}$.

Proof. i) Clearly $\rho_{1} \rho_{2}$ is localized in $\mathscr{S}_{0}$. It follows then from (iii) that $\rho_{1} \rho_{2} \in(S)$, hence $\rho_{1} \rho_{2} \in \Delta_{\mathscr{S}_{0}}$.

ii) If $\hat{\mathscr{S}}_{a}$ is an auxiliary cone with $\hat{\mathscr{S}}_{a} \subset \mathscr{S}_{a} \subset \mathscr{S}_{0}^{\prime}$ then $\rho_{1}^{\hat{\mathscr{S}}_{a}}$ is an extension of $\rho_{1}^{\mathscr{P}_{a}}$. But according to the previous lemma one has $\rho_{2}(\mathfrak{U}) \subset \mathfrak{B}^{\mathscr{S}_{a}}$ and therefore $\rho_{1}^{\hat{\mathscr{P}}_{a}} \rho_{2}=$ $\rho_{1}^{\mathscr{S}}{ }_{a} \rho_{2}$ on $\mathfrak{U}$. For arbitrary $\hat{\mathscr{S}}_{a}$ one chooses an interpolating sequence of spacelike cones $\mathscr{S}_{a}^{(i)} \subset \mathscr{S}_{0}^{\prime}, i=1, \ldots n$ such that $\mathscr{S}_{a}^{(1)}=\mathscr{S}_{a}, \mathscr{S}_{a}^{(n)}=\hat{\mathscr{S}}_{a}$ and either $\mathscr{S}_{a}^{(i)} \subset \mathscr{S}_{a}^{(i+1)}$ 
or $\mathscr{S}_{a}^{(i)} \supset \mathscr{S}_{a}^{(i+1)}, i=1, \cdots n-1$. The result follows then after repeated application of the previous argument.

iii) First one considers the case where $\hat{\mathscr{S}}_{0} \subset \mathscr{S}_{0}$. Let $V_{i}$ be unitary intertwiners from $\rho_{i}$ to $\hat{\rho}_{i}, i=1,2$. Then $V_{i} \in \mathfrak{A}\left(\mathscr{S}_{0}\right)^{-}, i=1,2$ according to relation (4.6), and since $\mathfrak{U}\left(\mathscr{S}_{0}\right)^{-} \subset \mathfrak{B}^{\mathscr{S}_{a}}$, one gets for any $A \in \mathfrak{U}$

$$
\begin{aligned}
\hat{\rho}_{1} \hat{\rho}_{2}(A) & =\hat{\rho}_{1}^{\mathscr{S}_{a} \circ} \hat{\rho}_{2}(A)=V_{1} \rho_{1}^{\mathscr{S}_{a}}\left(V_{2} \rho_{2}(A) V_{2}^{-1}\right) V_{1}^{-1} \\
& =V_{1} \rho_{1}^{\mathscr{S}_{a}}\left(V_{2}\right) \rho_{1} \rho_{2}(A) \rho_{1}^{\mathscr{S}_{a}}\left(V_{2}^{-1}\right) V_{1}^{-1} .
\end{aligned}
$$

Hence in this case $\rho_{1} \rho_{2} \simeq \hat{\rho}_{1} \hat{\rho}_{2}$. The result for a general $\hat{\mathscr{S}}_{0}$ follows again by choosing a sequence of interpolating cones and applying this argument repeatedly.

QED.

If $\mathscr{S}_{1}, \cdots \mathscr{S}_{n}$ are spacelike cones in the spacelike complement of $\mathscr{S}_{a}$ and $\rho_{i} \in \Delta_{\mathscr{S}_{1}}$, $i=1, \cdots n$, one may also consider the representation $\rho_{1}^{\mathscr{S}_{a}} \cdots \rho_{n-1}^{\mathscr{S}_{a}}{ }^{a} \rho_{n}$. But this representation is equivalent to some $\rho \in \Delta_{\mathscr{S}_{0}}$, so the set of sectors is not enlarged by this representation. We may therefore restrict our attention to products of morphisms with a common localization cone.

To conclude this section we note commutation properties of the morphisms $\rho$ which are analogous to results in [DHR I].

Proposition 4.3. Let $\mathscr{S}_{1}$ and $\mathscr{S}_{2}$ be spacelike separated cones in the spacelike complement of some $\mathscr{S}_{a}$, and let $\rho_{i} \in \Delta_{\mathscr{S}_{i}}, i=1,2$. Then $\rho_{1}^{\mathscr{S}_{a} \circ} \rho_{2}^{\mathscr{S}_{a}}=\rho_{2}^{\mathscr{S}_{a} \circ} \rho_{1}^{\mathscr{S}_{a}}$ on $\mathfrak{B}^{\mathscr{S}_{a}}$.

Proof. Since $\rho_{1}^{\mathscr{S}_{a} \circ} \rho_{2}^{\mathscr{S}_{a}}$ and $\rho_{2}^{\mathscr{S}_{a} \circ} \rho_{1}^{\mathscr{S}_{a}}$ are weakly continuous on $\mathfrak{A}^{c}\left(\mathscr{S}_{a}+x\right)^{-}$for each $x$, it suffices to verify the statement for local observables. Now for $A \in \mathfrak{A}(\mathcal{O})$

$$
\begin{aligned}
& \rho_{1}^{\mathscr{S}_{a} \circ} \rho_{2}^{\mathscr{S}_{a}}(A)=\rho_{1}^{\mathscr{S}_{a} \circ} \rho_{2}(A)=\rho_{1}^{\mathscr{S}_{1} \circ} \rho_{2}(A), \\
& \rho_{2}^{\mathscr{S}_{a} \circ} \rho_{1}^{\mathscr{S}_{a}}(A)=\rho_{2}^{\mathscr{S}_{a} \circ} \rho_{1}(A)=\rho_{2}^{\mathscr{S}_{2} \circ} \rho_{1}(A),
\end{aligned}
$$

hence if $V_{i}$ is a unitary intertwiner from $\rho_{i}$ to $\hat{\rho}_{i} \in \Delta_{\hat{\mathscr{S}}_{2}}, \hat{\mathscr{S}}_{i} \subset \mathscr{S}_{i} \cap \mathcal{O}^{\prime}, i=1,2$ then $V_{i} \in \mathfrak{A}\left(\mathscr{S}_{i}\right)^{-}, i=1,2$ and

$$
\rho_{1}^{\mathscr{S}_{1} \circ \rho_{2}}(A)=V_{1}^{-1} V_{2}^{-1} A V_{2} V_{1}=V_{2}^{-1} V_{1}^{-1} A V_{1} V_{2}=\rho_{2}^{\mathscr{S}_{2} \circ \rho_{1}}(A) . \quad \text { QED. }
$$

It follows from this proposition that the equivalence class of $\rho_{1} \rho_{2}, \rho_{1}, \rho_{2} \in \Delta_{\mathscr{S}_{0}}$ does not depend on the order of factors.

\section{Construction of Fields}

We have seen in the previous section that the morphisms $\rho$ can be composed, provided one extends them in a suitable manner. In order to simplify the calculations with these morphisms we introduce the concept of a field bundle which is intrinsically defined in our setting and has many structural properties in common with an algebra of field operators. This formalism has been invented by Doplicher, Haag and Roberts [DHR II], and we adopt here their notation and conventions with minor modifications.

The vector states in a representation $\rho \in \Delta_{\mathscr{S}_{0}}$ are described in this formalism 
by pairs $\boldsymbol{\Psi}=\{\rho, \Psi\} \in \Delta_{\mathscr{S}_{0}} \times \mathscr{H}_{0}$, which one interprets as elements of some vector bundle $\mathscr{H}$ with fibers labeled by $\rho$. Within each fiber $\mathscr{H}_{\rho}$ of $\mathscr{H}$ one has a scalar product

$$
(\{\rho, \Psi\},\{\rho, \Phi\})=(\Psi, \Phi)
$$

where $(\Psi, \Phi)$ is the scalar product in $\mathscr{H}_{0}$. Generalized field operators are pairs $\boldsymbol{B}=\{\rho, B\} \in \Delta_{\mathscr{S}_{0}} \times \mathfrak{B}^{\mathscr{S}_{a}}$ where $\mathscr{S}_{a} \subset \mathscr{S}_{0}^{\prime}$, and they act on $\mathscr{H}$ according to

$$
\{\rho, B\} \cdot\left\{\rho_{1}, \Psi\right\}=\left\{\rho_{1} \rho, \rho_{1}^{\mathscr{S}_{a}}(B) \Psi\right\}
$$

so they interpolate between different fibers. A norm of the generalized fields is given by

$$
\|\{\rho, B\}\|=\|B\|
$$

We call $\mathfrak{F}^{\mathscr{S}_{a}}=\Delta_{\mathscr{S}_{0}} \times \mathfrak{B}^{\mathscr{S}_{a}}$ a field bundle ${ }^{10}$. Clearly one has $\mathfrak{F}^{\mathscr{S}_{a}+x}=\mathfrak{F}^{\mathscr{S}_{a}}$ since $\mathfrak{B}^{\mathscr{S}_{a}+x}=\mathfrak{B}^{\mathscr{S}_{a}}$ and $\rho^{\mathscr{S}_{a}+x}=\rho^{\mathscr{S}_{a}}$ for all $\rho \in \Delta_{\mathscr{S}_{0}}$. The observables are embedded into the field bundles as pairs $A=\{l, A\}$, where $A \in \mathfrak{A}$ and $\iota$ is the identity automorphism of $\mathfrak{A}$, so in particular

$$
(\{\rho, \Psi\}, \boldsymbol{A}\{\rho, \Psi\})=(\Psi, \rho(A) \Psi) .
$$

It is also convenient to introduce intertwiners $\boldsymbol{T}=\left(\rho_{1}|T| \rho\right)$ on $\mathscr{H}$ as mappings between fibers corresponding to non-disjoint representations $\rho$ and $\rho_{1}$. If $\rho_{1}(A) T=T \rho(A), A \in \mathfrak{U}$ then

$$
\left(\rho_{1}|T| \rho\right) \cdot\{\rho, \Psi\}=\left\{\rho_{1}, T \Psi\right\} .
$$

One checks easily that intertwiners and observables commute. In Sect. 6 we will introduce the notion of an adjoint of a generalized field and in Sect. 7 we will define translations on $\mathfrak{F}^{\mathscr{S}_{a}}$.

Using the localization properties of the morphisms $\rho$ one can introduce a local structure on the field bundles $\mathfrak{F}^{\mathscr{S}_{a}}$. It is natural to assign the generalized field operator $\{\rho, B\}$ to the cone $\mathscr{S}_{0}$ if $\rho \in \Delta_{\mathscr{S}_{0}}$ and $B \in \mathfrak{U}\left(\mathscr{S}_{0}\right)^{-}$. For the identification of fields in a bundle $\mathfrak{F}^{\mathscr{S}_{a}}$ with different localization properties, one makes use of the fact that the above formalism leads to a redundant description of physical states: it follows from relation (5.4) that the vectors $\{\rho, \Psi\}$ and $\left\{\rho_{1}, V \Psi\right\}$ (which may belong to different vector bundles) induce the same physical state on $\mathfrak{A}$ if $\rho$ and $\rho_{1}$ are equivalent with $V$ as a unitary intertwiner. Similarly, the generalized fields $\{\rho, B\}$ and $\left\{\rho_{1}, V B\right\}$ (which may act on different vector bundles) induce the same action on physical states. Taking this into account one is led to the following

Definition. Let $\mathscr{S} \subset \mathscr{S}_{a}^{\prime}$ be any spacelike cone. The generalized field $\{\rho, B\} \in \mathfrak{F}^{\mathscr{S}_{a}}$ is said to be localized in $\mathscr{S}$, if for each $\rho_{1} \in \Delta_{\mathscr{S}}$ which is unitarily equivalent to $\rho$ with $V$ as intertwiner one has $V B \in \mathfrak{A}(\mathscr{S})^{-}$. The set of generalized fields which are localized in $\mathscr{S}$ is denoted by $\mathfrak{F}(\mathscr{S})$.

10 Note that in contrast to the situation in [DHR II] there exist many vector bundles $\mathscr{H}$ in the present case which are distinguished by localization cones $\mathscr{S}_{0}$, and on a fixed vector bundle $\mathscr{H}$ act several field bundles $\mathfrak{F}^{\mathscr{S}_{a}}$ corresponding to different auxiliary cones $\mathscr{S}_{a}$ 
We remark that the generalized fields in $\mathfrak{F}(\mathscr{S})$ commute with all observables $\{l, A\}$ if $A \in \mathfrak{H}^{c}(\mathscr{S})$. Because of duality the converse is also true: any generalized field in $\mathfrak{F}^{\mathscr{S}_{a}}$ which commutes with all these observables is an element of $\mathfrak{F}(\mathscr{S})$. But one has to mind that for fixed $\mathscr{S}_{a}$ there exist only generalized fields in the field bundle $\mathfrak{F}^{\mathscr{S}_{a}}$ which are localized in cones $\mathscr{S} \subset \mathscr{S}^{\prime}+x$. This apparent difficulty is resolved in the subsequent proposition where we will show that the set $\mathfrak{F}(\mathscr{S})$ of generalized fields which are localized in some $\mathscr{S}$ does not depend on $\mathscr{S}_{a}$. Phrased differently, $\mathfrak{F}(\mathscr{S})$ can be regarded as a subset of any field bundle $\mathfrak{F}^{\mathscr{S}_{a}}$ with $\mathscr{S}_{a} \subset \mathscr{S}^{\prime}$

Proposition 5.1. $\mathfrak{F}(\mathscr{S})$ is independent of the choice of the auxiliary cone $\mathscr{S}_{a} \subset \mathscr{S}_{0}^{\prime} \cap \mathscr{S}^{\prime}$.

Proof. Let $\hat{\mathscr{S}}_{a} \subset \mathscr{S}_{0}^{\prime} \cap \mathscr{S}^{\prime}$ be any spacelike cone. If $\{\rho, B\} \in \mathfrak{F}(\mathscr{S})$ there exists a unitary intertwiner $V$ from $\rho \in \Delta_{\mathscr{S}_{0}}$ to some $\rho_{1} \in \Delta_{\mathscr{S}}$ such that $V B \in \mathfrak{U}(\mathscr{S})^{-} \subset$ $\mathfrak{A}^{c}\left(\hat{\mathscr{S}}_{a}\right)^{-}$, and (using relation (4.6)) $V \in \mathfrak{Q}^{c}\left(\hat{\mathscr{S}}_{a}\right)^{-}$. So $B \in \mathfrak{B}^{\hat{\mathscr{Y}}_{a}}$ and consequently $\{\rho, B\} \in \mathfrak{F}^{\hat{\mathscr{S}}_{a}}$. It remains to show that the action of $\{\rho, B\}$ on $\mathscr{H}$ is independent of the choice of the auxiliary cone, i.e. $\check{\rho}^{\mathscr{S}_{a}}(B)=\check{\rho}^{\hat{\mathscr{S}}_{a}}(B)$ for every $\check{\rho} \in \Delta_{\mathscr{S}_{0}}$. If $\hat{\mathscr{S}}_{a} \subset \mathscr{S}_{a}$ or $\mathscr{S}_{a} \subset \hat{\mathscr{S}}_{a}$ this is trivial. But in four space-time dimensions any two spacelike cones $\mathscr{S}_{a}, \hat{\mathscr{S}}_{a} \subset \mathscr{S}_{0}^{\prime} \cap \mathscr{S}^{\prime}$ can be connected by an alternating sequence of spacelike cones $\mathscr{S}_{a}^{(i)} \subset \mathscr{S}_{0}^{\prime} \cap \mathscr{S}^{\prime}, \mathrm{i}=1, \ldots n$ (cf. the proof of Theorem 4.2), so the statement follows. QED.

We shall determine now the commutation relations between generalized fields which are localized in spacelike separated cones. These relations will be the basis for our discussion of statistics in the following section. Let $\boldsymbol{B}_{i}=\left\{\rho_{i}, B_{i}\right\} \in \mathfrak{F}\left(\mathscr{S}_{i}\right)$, $i=1,2$, where $\mathscr{S}_{1}, \mathscr{S}_{2}$ are such that $\mathscr{S}_{1} \subset \mathscr{S}_{2}^{\prime}$ and $\mathscr{S}_{0}^{\prime} \cap \mathscr{S}_{1}^{\prime} \cap \mathscr{S}_{2}^{\prime}$ contains some spacelike cone $\mathscr{S}_{a}$. Then there exist unitary intertwiners $V_{i}$ from $\rho_{i} \in \Delta_{\mathscr{S}_{0}}$ to $\hat{\rho}_{i} \in \Delta_{\mathscr{S}_{i}}$ for which $V_{i} B_{i}=C_{i} \in \mathfrak{A}\left(\mathscr{S}_{i}\right)^{-}$and

$$
\begin{aligned}
& \boldsymbol{B}_{1} \boldsymbol{B}_{2}=\left\{\rho_{2} \rho_{1}, \rho_{2}^{\mathscr{S}_{a}}\left(B_{1}\right) B_{2}\right\}=\left\{\rho_{2} \rho_{1}, \rho_{2}^{\mathscr{S}_{a}}\left(V_{1}^{-1}\right) V_{2}^{-1} C_{1} C_{2}\right\}, \\
& \boldsymbol{B}_{2} \boldsymbol{B}_{1}=\left\{\rho_{1} \rho_{2}, \rho_{1}^{\mathscr{S}_{a}}\left(V_{2}^{-1}\right) V_{1}^{-1} C_{2} C_{1}\right\} .
\end{aligned}
$$

From locality of the observables it follows that $C_{1} C_{2}=C_{2} C_{1}$. Furthermore, $V_{1} \rho_{1}^{\mathscr{S}_{a}}\left(V_{2}\right)$ is an intertwiner from $\rho_{1} \rho_{2}$ to $\hat{\rho}_{1}^{\mathscr{S}_{a}} \hat{\rho}_{2}$ and similarly $\rho_{2}^{\mathscr{S}_{a}}\left(V_{1}^{-1}\right) V_{2}^{-1}$ from $\hat{\rho}_{2}^{\mathscr{S} a_{\circ} \circ} \hat{\rho}_{1}$ to $\rho_{2} \rho_{1}$. But according to Proposition 4.3 we have $\hat{\rho}_{2}^{\mathscr{S} a \circ} \hat{\rho}_{1}=$ $\hat{\rho}_{1}^{\mathscr{S} a \circ} \hat{\rho}_{2}$, so

$$
\varepsilon\left(\rho_{1}, \rho_{2}\right)=\rho_{2}^{\mathscr{S}_{a}}\left(V_{1}^{-1}\right) V_{2}^{-1} V_{1} \rho_{1}^{\mathscr{S}_{a}}\left(V_{2}\right)
$$

is an intertwiner from $\rho_{1} \rho_{2}$ to $\rho_{2} \rho_{1}$. Denoting the action of $\boldsymbol{T}=\left(\rho_{1}|T| \rho\right)$ on a field bundle by

$$
\left(\rho_{1}|T| \rho\right) \circ\{\rho, B\}=\left\{\rho_{1}, T B\right\},
$$

(provided the right hand side is defined) we arrive at the commutation relations

$$
\boldsymbol{B}_{1} \boldsymbol{B}_{2}=\boldsymbol{\varepsilon}\left(\rho_{1}, \rho_{2}\right) \circ \boldsymbol{B}_{2} \boldsymbol{B}_{1},
$$

with $\varepsilon\left(\rho_{1}, \rho_{2}\right)=\left(\rho_{2} \rho_{1}\left|\varepsilon\left(\rho_{1}, \rho_{2}\right)\right| \rho_{1} \rho_{2}\right)$. Relation (5.9) implies that $\varepsilon\left(\rho_{1}, \rho_{2}\right)$ does not depend on the choice of the unitary intertwiners $V_{1}$ and $V_{2}$, and therefore $\varepsilon\left(\rho_{1}, \rho_{2}\right)$ does not change if one replaces $\mathscr{S}_{i}$ by $\hat{\mathscr{S}}_{i} \subset \mathscr{S}_{i}, i=1,2$. This fact together 
with Proposition 5.1 shows that $\varepsilon\left(\rho_{1}, \rho_{2}\right)$ is independent of the choice of the cones $\mathscr{S}_{1}, \mathscr{S}_{2}$ and $\mathscr{S}_{a}$, as long as $\mathscr{S}_{1} \subset \mathscr{S}_{2}^{\prime}$ and $\mathscr{S}_{a} \subset \mathscr{S}_{0}^{\prime} \cap \mathscr{S}_{1}^{\prime} \cap \mathscr{S}_{2}^{\prime}$.

The generalization of (5.9) to $n$-fold products is straightforward. Let $\boldsymbol{B}_{i}=$ $\left\{\rho_{i}, B_{i}\right\} \in \mathfrak{F}\left(\mathscr{S}_{i}\right), \mathscr{S}_{i} \subset \mathscr{S}_{j}^{\prime}, i, j=1, \ldots n, i \neq j$, and let $\mathscr{S}_{a} \subset \mathscr{S}_{0}^{\prime} \cap \mathscr{S}^{\prime} \cap \ldots \cap \mathscr{S}_{n}^{\prime}$. Then by repeated use of (5.9) one obtains the following relation for any permutation $p$ of $\{1, \ldots n\}$ :

$$
\boldsymbol{B}_{p^{-1}(n)} \ldots \boldsymbol{B}_{p^{-1}(1)}=\boldsymbol{\varepsilon}_{p}\left(\rho_{1}, \ldots \rho_{n}\right){ }^{\circ} \boldsymbol{B}_{n} \ldots \boldsymbol{B}_{1},
$$

where $\varepsilon_{p}\left(\rho_{1}, \ldots \rho_{n}\right)=\left(\rho_{p^{-1}(1)} \ldots \rho_{p^{-1}(n)}\left|\varepsilon_{p}\left(\rho_{1}, \ldots \rho_{n}\right)\right| \rho_{1} \ldots \rho_{n}\right)$. The unitary intertwiner $\varepsilon_{p}\left(\rho_{1}, \ldots \rho_{n}\right)$ is uniquely fixed by $(5.10)$ and does not depend on the choice of the cones $\mathscr{S}_{1}, \ldots \mathscr{S}_{n}$ and $\mathscr{S}_{a}$ within the above restrictions. The properties of the permutation operators $\varepsilon_{p}\left(\rho_{1}, \ldots \rho_{n}\right)$ follow easily from (5.10) (compare [DHR I, Thm. 4.2 and 4.3]). In the special case $\rho_{1}=\ldots=\rho_{n}=\rho$ we adopt the notation

$$
\varepsilon_{p}(\underbrace{\rho, \ldots \rho}_{n \text {-times }})=\varepsilon_{\rho}^{(n)}(p) .
$$

Then the analogue of [DHR I, Prop. 4.4] is

Theorem 5.2. Let $\rho \in \Delta_{\mathscr{S}_{0}}$. Then i) $p \rightarrow \varepsilon_{\rho}^{(n)}(p)$ is a unitary representation of the permutation group $P^{(n)}$. ii) $\varepsilon_{\rho}^{(n)}(p)$ commutes with $\rho^{n}(\mathfrak{U})$. iii) The equivalence class of the representation $\varepsilon_{\rho}^{(n)}$ of $P^{(n)}$ depends, for every $n \in \mathbb{N}$, only on the equivalence class of $\rho$.

Proof. (i) and (ii) are immediate consequences of (5.10). To prove (iii) let $\hat{\rho} \in \Delta_{\mathscr{S}_{0}}$ be unitarily equivalent to $\rho$. Then by explicit computation one can verify that $\varepsilon_{\hat{\rho}}^{(n)}$ is unitarily equivalent to $\varepsilon_{\rho}^{(n)}$. Again this result extends to morphisms $\hat{\rho} \simeq \rho$ with arbitrary localization cones by geometrical arguments. QED.

\section{Charge Conjugation and Statistics}

We use now the information on the intertwiners $\varepsilon_{\rho}^{(n)}(p)$ for an analysis of the possible statistics of the sectors $\rho$ and for the construction of charge conjugate sectors. A most familiar situation from field theory is present if $\rho^{2}$ is an irreducible representation. Then it follows from Theorem 5.2 that $\varepsilon(\rho, \rho)=: \varepsilon_{\rho}$ is a multiple of 1 , and since $\varepsilon_{\rho}^{2}=1$ because of relation (5.9) this multiple can only be 1 or -1 . This means that the generalized fields $\left\{\rho, B_{1}\right\}$ and $\left\{\rho, B_{2}\right\}$ commute, respectively anticommute, if they are localized in spacelike separated cones. We refer to this fact by saying that the sector $\rho$ has Bose, respectively Fermi, statistics. In both cases a conjugate sector can easily be constructed in view of

Proposition 6.1. Let $\rho \in \Delta_{\mathscr{S}_{0}}$ be an irreducible representation. Then the following statements are equivalent.

i) $\rho^{2}$ is irreducible, ii) $\varepsilon_{\rho}= \pm 1$, iii) $\rho^{\mathscr{S}_{a}}$ is an automorphism of $\mathfrak{B}^{\mathscr{S}_{a}}$ if $\mathscr{S}_{a} \subset \mathscr{S}_{0}^{\prime}$.

Proof. (iii) $\rightarrow$ (i) is obvious, and (i) $\rightarrow$ (ii) follows from Theorem 5.2 as explained above. It remains to establish (ii) $\rightarrow$ (iii). Let $B \in \mathfrak{U}^{c}\left(\mathscr{S}_{a}\right)^{-}$. Then we fix a cone $\hat{\mathscr{S}}_{a} \subset \mathscr{S}_{a}$ which is sufficiently small such that there exist cones $\mathscr{S}_{1} \subset \mathscr{S}_{a} \cap \hat{\mathscr{S}}_{a}^{\prime}$. 
Picking a $\rho_{1} \in \Delta_{\mathscr{S}_{1}}$ which is unitarily equivalent to $\rho$ with $V \in \mathfrak{B}^{\mathscr{S}_{a}}$ as intertwiner we get

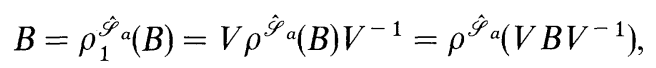

where we used that $V=\rho^{\hat{\mathscr{S}}_{a}}(V) \varepsilon_{\rho}$ according to relation (5.7) and $\varepsilon_{\rho}= \pm 1$ by assumption. Now the operator $V B V^{-1}$ is independent of the choice of $\mathscr{S}_{1}$ within the above restrictions since $\rho^{\hat{\mathscr{S}}_{a}}$ is isometric. So if $\mathcal{O} \subset \mathscr{S}_{a}$ is any bounded region we can choose $\mathscr{S}_{1} \subset \mathcal{O}^{\prime}$ and since $V \in\left(\mathfrak{U}^{c}\left(\mathscr{S}_{0}\right) \cap \mathfrak{U}^{c}\left(\mathscr{S}_{1}\right)\right)^{\prime} \subset \mathfrak{U}(\mathcal{O})^{\prime}$, we conclude that $V B V^{-1} \in \mathfrak{U}(\mathcal{O})^{\prime}$ for any $\mathcal{O} \subset \mathscr{S}_{a}$. This means that $V B V^{-1} \in \mathfrak{A}\left(\mathscr{S}_{a}\right)^{\prime}=\mathfrak{A}^{c}\left(\mathscr{S}_{a}\right)^{-}$ $\subset \mathfrak{B}^{\mathscr{S}_{a}}$, hence

$$
\mathfrak{A}^{c}\left(\mathscr{S}_{a}\right)^{-} \subset \rho^{\hat{\mathscr{S}}_{a}}\left(\mathfrak{H}^{c}\left(\mathscr{S}_{a}\right)^{-}\right)=\rho^{\mathscr{S}_{a}}\left(\mathfrak{H}^{c}\left(\mathscr{S}_{a}\right)^{-}\right) .
$$

Taking into account that $\rho^{\mathscr{S}_{a}}=\rho^{\mathscr{S}_{a}+x}$ it follows also that $\mathfrak{U}^{c}\left(\mathscr{S}_{a}+x\right)^{-} \subset$

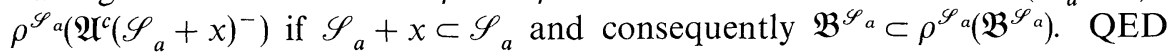

So in this situation a representation which is conjugate to $\rho$ can be obtained by restricting the inverse of $\rho^{\mathscr{S}_{a}}$ to $\mathfrak{A}$. It can easily be seen that the resulting representation $\bar{\rho}$ is again an element of $\Delta_{\mathscr{S}_{0}}$ and that $\bar{\rho} \rho$ is the vacuum representation. Also, the sector of $\bar{\rho}$ depends only on the sector of $\rho$.

We turn now to the more tedious analysis of the cases where the sector of $\rho$ has para-statistics. Then $\rho^{\mathscr{S}_{a}}$ does not have an inverse, but there always exist left-inverses of $\rho^{\mathscr{S}_{a}}$ if $\mathscr{S}_{a} \subset \mathscr{S}_{0}^{\prime}$, as we shall see below. A left-inverse of $\rho^{\mathscr{S}_{a}}, \mathscr{S}_{a} \subset \mathscr{S}_{0}^{i}$ is a positive linear mapping $\phi$ from $\mathfrak{B}^{\mathscr{S}_{a}}$ into $\mathscr{B}\left(\mathscr{H}_{0}\right)$ such that for $B_{i} \in \mathfrak{B}^{\mathscr{S}_{a}}, i=1,2,3$

$$
\begin{aligned}
\phi\left(\rho^{\mathscr{S}_{a}}\left(B_{1}\right) B_{2} \rho^{\mathscr{S}_{a}}\left(B_{3}\right)\right) & =B_{1} \phi\left(B_{2}\right) B_{3}, \\
\phi(1) & =1 .
\end{aligned}
$$

It follows from these conditions that $\phi\left(\mathfrak{H}^{c}\left(\mathscr{S}_{a}\right)^{-}\right) \subset \mathfrak{U}\left(\mathscr{S}_{a}\right)^{\prime}$ hence $\phi\left(\mathfrak{B}^{\mathscr{S}_{a}}\right) \subset \mathfrak{B}^{\mathscr{S}_{a}}$, so left-inverses can be composed.

Now according to Theorem 5.2, $\varepsilon_{\rho}$ commutes with $\rho^{2}(\mathfrak{U})$, so $\phi\left(\varepsilon_{\rho}\right)$ commutes with $\rho(\mathfrak{U})$. Hence if $\rho$ is an irreducible representation, $\phi\left(\varepsilon_{\rho}\right)$ is a multiple of the identity,

$$
\phi\left(\varepsilon_{\rho}\right)=\lambda_{\rho} \cdot 1
$$

The number $\lambda_{\rho}$ is called the statistics parameter of $\rho$. For the determination of the possible values of $\lambda_{\rho}$ we can rely on the results of [DHR I]. In that analysis it is only used that for each $n \in \mathbb{N} \phi^{n-1}$ induces a state on $\rho^{n}(\mathfrak{U})^{\prime} \subset \mathfrak{U}^{c}\left(\mathscr{S}_{0}\right)^{\prime}$ (which follows from the definition of $\phi$ ) and that this state is faithful if $\lambda_{\rho} \neq 0$ (this will be shown below). Using the same arguments as in [DHR I], one finds that the admissible values of $\lambda_{\rho}$ are $\pm \frac{1}{d}, d \in \mathbb{N}$ and 0 , and since the set of left-inverses of $\rho^{\mathscr{S}_{a}}$ is convex it follows also that $\lambda_{\rho}$ does not depend on the choice of $\phi$.

The value of $\lambda_{\rho}$ determines uniquely the equivalence class of the representations $\varepsilon_{\rho}^{(n)}$ of $P^{(n)}$ for every $n \in \mathbb{N}$. If $\lambda_{\rho}=\frac{1}{d}$ then the Young tableaux associated with the representation $\varepsilon_{\rho}^{(n)}$ are all tableaux with at most $d$ rows, so $\rho$ has para-Bose statistics 
of order $d$. If $\lambda_{\rho}=-\frac{1}{d}$ then all Young tableaux with at most $d$ columns appear, and $\rho$ has para-Fermi statistics of order $d$. If $\lambda_{\rho}=0$ there are no restrictions on the Young tableaux; this is the so-called infinite statistics case. For a derivation of these results see [DHR I].

So far we have considered in our analysis of statistics only representations $\rho \in \Delta_{\mathscr{S}_{0}}$ for which $\phi\left(\varepsilon_{\rho}\right)$ is a multiple of the identity. This is always the case if $\rho$ is an irreducible representation. However, since the set of irreducible representations in $\Delta_{\mathscr{S}_{0}}$ is (in the para-statistics case) not stable under composition we extend now our analysis to arbitary representations. Again, we generalize methods developed in $[$ DHR I] to the present situation. To this end we need the following positivity property of left-inverses $\phi$ of $\rho^{\mathscr{S}_{a}}$ :

$$
\phi\left(B^{*} B\right) \geqq \phi\left(B^{*}\right) \phi(B) \quad \text { for } \quad B \in \mathfrak{B}^{\mathscr{S}_{a}} .
$$

This property follows from the fact that the representation $\pi_{\phi}{ }^{\circ} \rho^{\mathscr{S}_{a}}$ of $\mathfrak{B}^{\mathscr{S}_{a}}$, where $\pi_{\phi}$ is the representation of $\mathfrak{B}^{\mathscr{S}_{a}}$ induced by the state $\omega_{0}{ }^{\circ} \phi$, contains a subrepresentation which is equivalent to the vacuum representation of $\mathfrak{B}^{\mathscr{S}_{a}}$ (compare [DHR I, Lemma 3.5]). Using relation (6.3) one can derive the estimate

$$
\left\|\phi\left(B^{*} B\right)\right\| \geqq\left\|\phi\left(\varepsilon_{\rho}\right) B^{*} B \phi\left(\varepsilon_{\rho}\right)\right\| \quad \text { for } \quad B \in \mathfrak{B}^{\hat{\mathscr{Y}}_{a}},
$$

where $\hat{\mathscr{S}}_{a}$ is any spacelike cone with $\mathscr{S}_{a} \subset \hat{\mathscr{S}}_{a} \subset \mathscr{S}_{0}^{\prime}$ which is sufficiently large such that there exists some spacelike cone $\mathscr{S}_{1} \subset \hat{\mathscr{S}}_{a} \cap \mathscr{S}_{a}^{\prime}$. To verify this let $B \in$ $\mathfrak{U}^{c}\left(\hat{\mathscr{S}}_{a}+x\right)^{-}$and let $\rho_{1} \in \Delta_{\mathscr{S}_{1}+x}$ be equivalent to $\rho$ with $V \in \mathfrak{B}^{\mathscr{S}_{a}}$ as intertwiner. Then

$$
V^{-1} B=V^{-1} \rho_{1}^{\mathscr{S}_{a}}(B)=\rho^{\mathscr{S}_{a}}(B) V^{-1}=\rho^{\mathscr{S}_{a}}(B) \varepsilon_{\rho} \rho^{\mathscr{S}_{a}}\left(V^{-1}\right),
$$

where we used relation (5.7). So by the defining properties of a left-inverse

$$
\phi\left(V^{-1} B\right)=B \phi\left(\varepsilon_{\rho}\right) V^{-1} .
$$

Now because of relation $(6.3)$ we have

$$
\phi\left(B^{*} B\right) \geqq \phi\left(B^{*} V\right) \phi\left(V^{-1} B\right)=V \phi\left(\varepsilon_{\rho}\right) B^{*} B \phi\left(\varepsilon_{\rho}\right) V^{-1},
$$

and taking the norm on both sides of this inequality the assertion follows.

As a first consequence of relation (6.4) we see that if $\phi\left(\varepsilon_{\rho}\right)=\lambda_{\rho} 1$ and $\lambda_{\rho} \neq 0$,

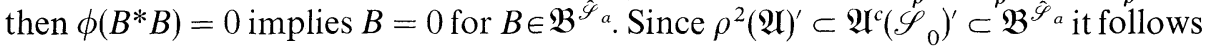
then that $\phi$ induces a faithful state on $\rho^{2}(\mathfrak{A})^{\prime}$ if $\rho$ is an irreducible representation with $\lambda_{\rho} \neq 0$; similarly $\phi^{n-1}$ induces a faithful representation of $\rho^{n}(\mathfrak{U})^{\prime}$, as was claimed above.

Next let $\rho$ be reducible, and let $E_{\delta}$ be the spectral projections of the selfadjoint operator $\phi\left(\varepsilon_{\rho}\right)$ corresponding to spectral values $\lambda$ with $|\lambda| \geqq \delta>0$. Since $E_{\delta} \in \rho(\mathfrak{A})^{\prime} \subset$ $\mathfrak{B}^{\hat{\mathscr{Y}} a}$ we get from relation (6.4)

$$
\left\|\phi\left(E_{\delta} B^{*} B E_{\delta}\right)\right\| \geqq \delta^{2} \cdot\left\|E_{\delta} B^{*} B E_{\delta}\right\|
$$

for every $B \in \mathfrak{B}^{\hat{\mathscr{Y}}_{a}}$. If in particular $E_{i} \in \rho(\mathfrak{A})^{\prime}, i=1, \ldots n$ is a set of mutually orthogonal projections with $E_{i} E_{\delta}=E_{i}$, then $\phi\left(E_{i}\right)$ is a multiple of the identity, so 
$\phi\left(E_{i}\right) \geqq \delta^{2} \cdot 1$. Therefore we can estimate

$$
1 \geqq \phi\left(\sum_{i=1}^{n} E_{i}\right)=\sum_{i=1}^{n} \phi\left(E_{i}\right) \geqq n \cdot \delta^{2} \cdot 1
$$

and consequently $n \leqq \delta^{-2}$. This shows that the spectrum of $\phi\left(\varepsilon_{\rho}\right)$ is discrete with the only possible accumulation point 0 . Moreover, $E_{\delta} \rho(\mathfrak{U})^{\prime} E_{\delta}$ is a finite, discrete von Neumann algebra, so if $\phi\left(\varepsilon_{\rho}\right) \neq 0$ the representation $\rho$ has irreducible subrepresentations. We shall show that these subrepresentations are localizable in cones and that they have finite statistics.

Let $E \in \rho(\mathfrak{U})^{\prime} \subset \mathfrak{A}\left(\mathscr{S}_{0}\right)^{-}$be a projection with $E \phi\left(\varepsilon_{\rho}\right)=\lambda \cdot E$ and $\lambda \neq 0$. Then, applying an argument of Borchers [24], one can find in any algebra $\mathfrak{A}\left(\hat{\mathscr{S}}_{0}\right)^{-}$, where $\hat{\mathscr{S}}_{0}$ is a slightly larger cone containing $\overline{\mathscr{S}}_{0}$ in its interior, an isometry $W$ with $W^{*} W=1$ and $W W^{*}=E$. Setting

$$
\rho_{E}(A)=W^{*} \rho(A) W \quad \text { for } A \in \mathfrak{A},
$$

one obtains a representation $\rho_{E} \in \Delta_{\hat{\mathscr{P}}_{0}}$ which is unitarily equivalent to the subrepresentation of $\rho$ acting on $E \mathscr{H}_{0}$. The statistics operator of $\rho_{E}$ can be computed and turns out to be

$$
\varepsilon_{\rho_{E}}=W^{*} \rho^{\mathscr{S}_{a}}\left(W^{*}\right) \varepsilon_{\rho} \rho^{\mathscr{S}_{a}}(W) W
$$

and a left-inverse of $\rho_{E}$ is given by

$$
\phi_{E}(B)=\phi\left(W B W^{*}\right) \cdot \phi(E)^{-1}
$$

(Note that $\phi(E)$ is a nonvanishing multiple of the identity.) Thus the statistics parameter $\lambda_{\rho_{\mathrm{E}}}$ of $\rho_{E}$ is obtained by the following calculation:

$$
\begin{aligned}
\phi_{E}\left(\varepsilon_{\rho_{E}}\right) & =\phi\left(W W^{*} \rho^{\mathscr{S}_{a}}\left(W^{*}\right) \varepsilon_{\rho} \rho^{\mathscr{S}_{a}}(W) W W^{*}\right) \phi(E)^{-1} \\
& =W^{*} \phi\left(E \varepsilon_{\rho} E\right) W \phi(E)^{-1} \\
& =W^{*} \phi\left(\rho^{\mathscr{S}_{a}}(E) \varepsilon_{\rho} \rho^{\mathscr{S}_{a}}(E)\right) W \phi(E)^{-1} \\
& =W^{*} E \phi\left(\varepsilon_{\rho}\right) E W \phi(E)^{-1} \\
& =\lambda \cdot \phi(E)^{-1} \neq 0
\end{aligned}
$$

where in the third equality we used the relation $\varepsilon_{\rho} E=\rho^{\mathscr{S} a}(E) \varepsilon_{\rho}$ which is a consequence of relation (6.5).

Hence we arrive at the conclusion that $\rho$ contains irreducible subrepresentations with finite statistics, whenever $\phi\left(\varepsilon_{\rho}\right) \neq 0$ for some left-inverse $\phi$. In particular, if $\rho$ is a factorial representation, it is either a multiple of an irreducible representation with finite statistics or $\phi\left(\varepsilon_{\rho}\right)=0$ for any left-inverse $\phi$. So it is no loss of generality to restrict the analysis of statistics to representations fulfilling relation (6.2).

We turn now to the question of the existence and uniqueness of left-inverses. In order to establish the existence of a left-inverse for each $\rho^{\mathscr{S}_{a}}$ we choose a sequence of cones $\mathscr{S}_{i}, i \in \mathbb{N}$ such that for any $x \in \mathbb{R}^{4}$ and for sufficiently large $k \in \mathbb{N}$ $\mathscr{S}_{k} \subset \mathscr{S}_{a}+x$. Then we pick morphisms $\rho_{i} \in \Delta_{\mathscr{S}_{i}}$ which are unitarily equivalent to $\rho$ with $V_{i}$ as intertwiners. Using the localization properties of the morphisms $\rho_{i}$ it is obvious that

$$
n-\lim _{i \rightarrow \infty} V_{i} \rho^{\mathscr{S}_{a}}(B) V_{i}^{-1}=B
$$


for $B \in \mathfrak{B}^{\mathscr{S}_{a}}$. So the sequence $V_{i}$ transfers the charge carried by $\rho^{\mathscr{S}_{a}}$ to spacelike infinity. As in [DHR I] we obtain now a left-inverse $\phi_{0}$ of $\rho^{\mathscr{S}_{a}}$ which is defined on $\mathfrak{B}^{\mathscr{S} a}$ by setting

$$
\sigma_{i}(B)=V_{i} B V_{i}^{-1}, \quad B \in \mathfrak{B}^{\mathscr{S}_{a}}
$$

and choosing a weak limit point $\phi_{0}$ of the sequence $\sigma_{i}$. (This means that for any $\Psi, \Psi^{\prime} \in \mathscr{H}_{0}$ and $B \in \mathfrak{B}^{\mathscr{S}_{a}}$ the matrix element $\left(\Psi, \phi_{0}(B) \Psi^{\prime}\right)$ is some limit point of the sequence $\left(\Psi, \sigma_{i}(B) \Psi^{\prime}\right)$. Such limit points $\phi_{0}$ exist due to compactness arguments [25].) Obviously, $\phi_{0}$ is a positive linear mapping from $\mathfrak{B}^{\mathscr{S}_{a}}$ into $\mathscr{B}\left(\mathscr{H}_{0}\right)$, and in view of relation (6.14) it satisfies also the defining condition (6.1) for left-inverses of $\rho^{\mathscr{I}_{a}}$.

If $\rho$ is a reducible representation of $\mathfrak{A}$ then the left-inverses of $\rho^{\mathscr{S}_{a}}$ are not unique. But if $\rho$ is irreducible and $\lambda_{\rho} \neq 0$, then there exists exactly one left-inverse $\phi_{0}$ of $\rho^{\mathscr{S}_{a}}$ which is normal on $\mathfrak{A}^{c}\left(\mathscr{\mathscr { S }}_{a}+x\right)^{-}$for any $x$. Such a $\phi_{0}$ is obtained as in the preceding discussion, but in order to gain control on the properties of $\phi_{0}$ we must choose the cones $\mathscr{S}_{i}$ in such a way that the intertwiners $V_{i}$, which are used in the construction of $\phi_{0}$, are in the domain of $\phi_{0}$. To this end we choose a sequence of auxiliary cones $\mathscr{S}_{a}^{(i)}, i \in \mathbb{N}$ which is such that $\mathscr{S}_{a}^{(i+1)} \subset \mathscr{S}_{a}^{(i)}$, and each region $\mathscr{S}_{a}^{(i)} \cap \mathscr{S}_{a}^{(i+1)^{\prime}}$ contains some spacelike cone. Then we pick a sequence of localization cones $\mathscr{S}_{i} \subset \mathscr{S}_{a}^{(i)} \cap \mathscr{S}_{a}^{(i+1)^{\prime}}$ with the property that for each $x$ there exists some sufficiently large $k \in \mathbb{N}$ for which $\mathscr{S}_{k} \subset \mathscr{S}_{a}^{(k)}+x$. With these preparations we achieve two things: first the intertwiners $V_{i}$ in relation (6.5) are contained in $\mathfrak{B}^{\mathscr{S}_{a}{ }^{(2+1)}}$, and second the left-inverses $\phi_{0}$ of $\rho^{\mathscr{S}_{a}}$ obtained this way can be extended to leftinverses of $\rho^{\mathscr{S}_{a}(i)}$ for any $i \in \mathbb{N}$. As a matter of fact, if

$$
\mathfrak{C}:=\bigcup_{i}^{c *} \mathfrak{B}^{\mathscr{S}_{a}(l)},
$$

then any weak limit point $\bar{\phi}_{0}$ of the sequence of isomorphisms $\bar{\sigma}_{i}: \bar{\sigma}_{i}(C)=V_{i} C V_{i}^{-1}$, $C \in \mathbb{C}$ has the following properties: it is a positive linear mapping of $\mathbb{C}$ into $\mathscr{B}\left(\mathscr{H}_{0}\right)$, and the restriction of $\bar{\phi}_{0}$ to any algebra $\mathfrak{B}^{\mathscr{S}_{a}^{(i)}}$ defines a left-inverse of $\rho^{\mathscr{S}_{a}^{(1)}}$. We shall call the left-inverses $\phi$ of $\rho^{\mathscr{S}_{a}}$ which have an extension $\bar{\phi}$ with these properties c-extendable.

In the next step we prove that there exists only one $\mathfrak{C}$-extendable left-inverse $\phi_{0}$ of $\rho^{\mathscr{S}_{a}}$ if $\lambda_{\rho} \neq 0$. Let $\phi$ be any $\mathfrak{C}$-extendable left-inverse of $\rho^{\mathscr{S}_{a}}$ and let $B \in \mathfrak{U}^{c}\left(\mathscr{S}_{a}+x\right)^{-}$. Then it follows from relation (6.7) that

$$
\phi\left(B^{*} B\right)=\bar{\phi}\left(B^{*} B\right) \geqq \lambda_{\rho}^{2} V_{i} B^{*} B V_{i}^{-1}
$$

for sufficiently large $i \in \mathbb{N}$. (Here one needs that the intertwiners $V_{i}$ are in the domain of $\bar{\phi}$.) So going to the limit of large $i$ one finds that

$$
\phi\left(B^{*} B\right) \geqq \lambda_{\rho}^{2} \phi_{0}\left(B^{*} B\right) \quad \text { for } \quad B \in \mathfrak{B}^{\mathscr{S}_{a}} .
$$

If $\lambda_{\rho}^{2}=1$ this leads immediately to the conclusion that $\phi$ and $\phi_{0}$ coincide on $\mathfrak{B}^{\mathscr{S}_{a}}$ because $\left(\phi-\phi_{0}\right)\left(B^{*} B\right) \geqq 0$ implies

$$
\left\|\left(\phi-\phi_{0}\right) \uparrow \mathfrak{B}^{\mathscr{S}_{a}}\right\|=\left\|\left(\phi-\phi_{0}\right)(1)\right\|=0 .
$$

If $\lambda_{\rho}^{2}<1$, then $\left(1-\lambda_{\rho}^{2}\right)^{-1} \cdot\left(\phi-\lambda_{\rho}^{2} \phi_{0}\right)$ is again a $\mathbb{C}$-extendable left-inverse of $\rho^{\mathscr{S}_{a}}$. 
So applying the estimate (6.18) we get for $B \in \mathfrak{B}^{\mathscr{S}_{a}}$

$$
\phi\left(B^{*} B\right) \geqq \lambda_{\rho}^{2} \cdot\left(1+\left(1-\lambda_{\rho}^{2}\right)\right) \phi_{0}\left(B^{*} B\right)
$$

and after $n$ steps

$$
\phi\left(B^{*} B\right) \geqq \lambda_{\rho}^{2} \cdot \sum_{i=0}^{n}\left(1-\lambda_{\rho}^{2}\right)^{i} \cdot \phi_{0}\left(B^{*} B\right) .
$$

Hence if $\lambda_{\rho} \neq 0$ we obtain in the limit $n \rightarrow \infty$ the desired result $\left(\phi-\phi_{0}\right) \uparrow \mathfrak{B}^{\mathscr{S}_{a}}=0$.

Now since all weak limit points of the sequence $\bar{\sigma}_{i} \uparrow \mathfrak{B}^{\mathscr{S}_{a}}$ defined above are C-extendable left-inverses of $\rho^{\mathscr{S}_{a}}$ (by construction), it follows that $\phi_{0}$ is the only weak limit point of this sequence. Therefore, since the algebras $\mathfrak{U}^{c}\left(\mathscr{S}_{a}+x\right)^{-} \subset \mathfrak{B}^{\mathscr{S}_{a}}$ are weakly closed, $\phi_{0}$ is normal on $\mathfrak{A}^{c}\left(\mathscr{S}_{a}+x\right)^{-}$for any $x[9]$.

Next one sees, using the above uniqueness result, that the restrictions of all left-inverses of $\rho^{\mathscr{S}_{a}}$ to the algebra $\mathfrak{A}$ coincide. This is so because if one restricts any left-inverse $\phi$ of $\rho^{\mathscr{S}_{a}}$ to an algebra $\mathfrak{B}^{\hat{\mathscr{S}}_{a}}$, where $\hat{\mathscr{S}}_{a} \supset \mathscr{S}_{a}$ is sufficiently large, then this restriction is $\hat{\mathbb{C}}$-extendable (for some suitable algebra $\hat{\mathbb{C}} \supset \mathfrak{B}^{\hat{\mathscr{S}}_{a}}$ ), and it

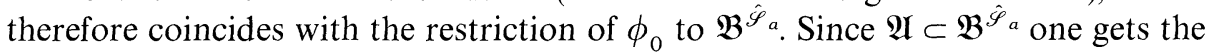
desired result $\left(\phi-\phi_{0}\right) \uparrow \mathfrak{U}=0$.

If in particular $\phi$ is normal on $\mathfrak{U}^{c}\left(\mathscr{S}_{a}+x\right)^{-}$for all $x$, then $\left(\phi-\phi_{0}\right) \uparrow \mathfrak{B}^{\mathscr{S}_{a}}=0$. So $\phi_{0}$ is the only left-inverse of $\rho^{\mathscr{S}_{a}}$ which is normal on the algebras $\mathfrak{A}^{c}\left(\mathscr{S}_{a}+x\right)^{-}$. Finally, choosing alternating sequences of interpolating cones (cf. the proof of Theorem 4.2) it follows that the restriction of $\phi_{0}$ to $\mathfrak{A}$ does not depend on the choice of the auxiliary cone $\mathscr{S}_{a}$. We collect these results in

Proposition 6.2. Let $\rho \in \Delta_{\mathscr{S}_{0}}$ be irreducible with finite statistics, $\lambda_{\rho} \neq 0$. Then for each spacelike cone $\mathscr{S}_{a} \subset \mathscr{S}_{0}^{\prime}$ there exists a unique left-inverse $\phi^{\mathscr{S}_{a}}$ of $\rho^{\mathscr{S}_{a}}$ which is normal on $\mathfrak{A}^{c}\left(\mathscr{S}_{a}+x\right)^{-}$for all $x$, and $\phi^{\mathscr{S}_{a}} \uparrow \mathfrak{U}$ does not depend on the choice of $\mathscr{S}_{a}$.

Using this result we can establish now the existence of representations $\bar{\rho}$ which are conjugate to $\rho$.

Theorem 6.3. Let $\rho \in \Delta_{\mathscr{S}_{0}}$ be irreducible with finite statistics. Then there exists an irreducible representation $\bar{\rho} \in \Delta_{\mathscr{S}_{0}}$ such that $\bar{\rho} \rho$ contains exactly once a subrepresentation which is equivalent to the vacuum representation. $\bar{\rho}$ is unique to within equivalence.

Proof. Let $\phi^{\mathscr{S}_{a}}$ be the left-inverse of $\rho^{\mathscr{S}_{a}}$ distinguished by the preceding proposition. Then the state $\omega_{\phi}=\omega_{0}{ }^{\circ} \phi^{\mathscr{S}_{a}} \uparrow \mathfrak{A}$ does not depend on $\mathscr{S}_{a} \subset \mathscr{S}_{0}^{\prime}$ and extends to a normal state on $\mathfrak{A}^{c}\left(\mathscr{S}_{a}+x\right)^{-}$for any $x$. This implies in particular that $\omega_{0}{ }^{\circ} \phi^{\mathscr{S}_{a}} \times$ $\left(V^{-1} A V\right), A \in \mathfrak{U}$ is for each unitary operator $V \in \mathfrak{B}^{\mathscr{S}_{a}}$ a vectorstate in the representation $\pi_{\phi}$ of $\mathfrak{A}$ induced by $\omega_{\phi}$. Now let $\mathscr{S}_{1}$ be any spacelike cone and let $\rho_{1} \in \Delta_{\mathscr{S}_{1}}$ be unitarily equivalent to $\rho$ with $V$ as an intertwiner. Then, choosing a cone $\mathscr{S}_{a} \subset$ $\mathscr{S}_{0}^{\prime} \cap \mathscr{S}_{1}^{\prime}$, one gets

$$
\omega_{0}{ }^{\circ} \phi^{\mathscr{S}_{a}}\left(V^{-1} A V\right)=\omega_{0}(A) \quad \text { if } A \in \mathfrak{U}^{c}\left(\mathscr{S}_{1}\right) .
$$

If $\pi_{\phi}$ is a positive energy representation, one can use this relation as in the proof of Theorem 3.5 to show that $\pi_{\phi}$ is localizable in spacelike cones. The remaining statements follow then from our uniqueness result on left-inverses. If $\pi_{\phi}$ is not 
known to be a positive energy representation, then one must apply the methods of [DHR II] for the construction of a $\bar{\rho} \in \Delta_{\mathscr{S}_{0}}$ with $\bar{\rho} \simeq \pi_{\phi}$. We remark that the latter construction makes the role of the finiteness of statistics for the result more transparent. QED.

It follows also from the analysis of [DHR II] that the statistics parameter of $\bar{\rho}$ coincides with the statistics parameter of $\rho$. For the sake of convenience we give here a direct calculation.

Proposition 6.4. Let $\rho \in \Delta_{\mathscr{S}_{0}}$ be irreducible with finite statistics, and let $\bar{\rho}$ be a conjugate representation. Then $\lambda_{\bar{\rho}}=\lambda_{\rho}$.

Proof. Let $R$ be an isometric intertwiner from the identical (vacuum) representation $l$ to $\bar{\rho} \rho$ where $\bar{\rho} \in \Delta_{\mathscr{S}_{0}}$ is irreducible and conjugate to $\rho$. Then, if $\mathscr{S}_{a} \subset \mathscr{S}_{0}^{\prime}$, the unique left-inverse $\phi_{\rho}^{\mathscr{S}_{a}}$ of $\rho^{\mathscr{S}_{a}}$ is given by the formula

$$
\phi_{\rho}^{\mathscr{S}_{a}}(B)=R^{*} \bar{\rho}^{\mathscr{S}_{a}}(B) R, \quad B \in \mathfrak{B}^{\mathscr{S}_{a}} .
$$

Now if $V$ is a unitary intertwiner from $\rho$ to some $\rho_{1} \in \Delta_{\mathscr{S}_{1}}, \mathscr{S}_{1} \subset \mathscr{S}_{0}^{\prime} \cap \mathscr{S}_{a}^{\prime}$, then

$$
\varepsilon_{\rho}=V^{-1} \rho^{\mathscr{S}_{a}}(V),
$$

and the statistics parameter $\lambda_{\rho}$ of $\rho$ can be expressed according to

$$
\begin{aligned}
\lambda_{\rho} 1 & =\phi_{\rho}^{\mathscr{S}_{a}}\left(\varepsilon_{\rho}\right)=R^{*} \bar{\rho}^{\mathscr{S}_{a}}\left(V^{-1} \rho^{\mathscr{S}_{a}}(V)\right) R=R^{*} \bar{\rho}^{\mathscr{S}_{a}}\left(V^{-1}\right)(\bar{\rho} \rho)^{\mathscr{S}_{a}}(V) R \\
& =R^{*} \bar{\rho}^{\mathscr{S}_{a}}\left(V^{-1}\right) R V=R^{*} \bar{\rho}^{\mathscr{S}_{a}}\left(V^{*}\right) V \rho^{\mathscr{S}_{a}}(R) \\
& =R^{*} \varepsilon(\rho, \bar{\rho}) \rho^{\mathscr{S}_{a}}(R),
\end{aligned}
$$

where we used that $R \in \mathfrak{H}^{c}\left(\mathscr{S}_{0}\right)^{\prime} \subset \mathfrak{A}\left(\mathscr{S}_{1}\right)^{\prime}$. Since $\varepsilon(\bar{\rho}, \rho) R$ is an isometric intertwiner from $l$ to $\rho \bar{\rho}$, we obtain by the same calculation the following expression for the statistics parameter $\lambda_{\bar{\rho}}$ of $\bar{\rho}$ :

$$
\lambda_{\bar{\rho}} 1=R^{*} \bar{\rho}^{\mathscr{\varphi}_{a}}(\varepsilon(\bar{\rho}, \rho) R) .
$$

Hence $\lambda_{\bar{\rho}} 1=\left(\lambda_{\bar{\rho}} 1\right)^{*}=R^{*}\left(\lambda_{\bar{\rho}} 1\right)^{*} R=R^{*} \bar{\rho}^{\mathscr{S} a}\left(R^{*} \varepsilon(\rho, \bar{\rho})\right) R R$

$$
=R^{*} \bar{\rho}^{\mathscr{S}_{a}}\left(R^{*} \varepsilon(\rho, \bar{\rho})\right)(\bar{\rho} \rho)^{\mathscr{S} a}(R) R=R^{*} \bar{\rho}^{\mathscr{S}_{a}}\left(R^{*} \varepsilon(\rho, \bar{\rho}) \rho^{\mathscr{S}_{a}}(R)\right) R=\lambda_{\rho} 1 .
$$

QED.

We remark that the machinery developed so far is sufficient to prove as in [DHR I] that the set of sectors with finite statistics is closed under the operations of composition of sectors, performing finite direct sums and taking subrepresentations and conjugates.

In the case of infinite statistics a reasonable conjugate sector cannot be constructed. It is therefore gratifying that for single particle representations finiteness of statistics can be proved. For irreducible single particle representations this has been done in [17], and we sketch here the straightforward generalization of this argument to factorial single particle representations.

The idea is to construct the conjugate sector directly by combining the information on the localization properties of single particle states (Theorem 3.1) with the fact that single particle representations are localizable in cones (Theorem 3.4). So let $\rho \in \Delta_{\mathscr{S}_{0}}$ be a single particle representation. Then we consider the follow- 
ing states in the vacuum sector:

$$
\varphi_{x}(A)=\left(U_{\rho}(x) B E\left(\Delta_{1}^{\sim}\right) \Phi, A U_{\rho}(x) B E\left(\Delta_{1}^{\sim}\right) \Phi\right), A \in \mathfrak{U} .
$$

Here $U_{\rho}(x)$ are the translations in the representation $\rho, B$ and $E\left(\Delta_{1}\right)$ are chosen according to Theorem 3.1 with respect to the representation $\rho$ and $\Phi \in \mathscr{H}_{0}$ with $\left\|B E\left(\Delta_{1}^{\sim}\right) \Phi\right\|=1$. Since $\rho$ acts trivially on $\mathfrak{A}^{c}\left(\mathscr{S}_{0}\right), \varphi_{x}$ looks for observables in $\mathfrak{U}^{c}\left(\mathscr{S}_{0}\right)$ like a single particle state which is localized in some region about $x$. On the other hand, $\varphi_{x}$ has total charge zero, so the charge of this particle is compensated in the state $\varphi_{x}$ within the cone $\mathscr{S}_{0}$.

Now let $x$ tend to infinity in the spacelike complement of $\mathscr{S}_{0}$ such that for some neighbourhood $\mathcal{O}$ of the origin $x+\lambda(x) \mathcal{O} \subset \mathscr{S}_{0}^{\prime}$, where $\lambda(x)$ is a positive function which tends (not too slowly) to infinity as $|x|$ becomes large. Then the effect of the original particle in the states $\varphi_{x}$ is shifted to infinity, and only the compensating charge remains. To verify this we apply Corollary 3.2, giving

$$
\left|\partial_{\mu} \varphi_{x}(B)\right| \leqq h(\lambda)\|B\|, B \in \rho(\mathfrak{A}(\lambda \mathcal{O}+x))^{\prime}
$$

with a rapidly decreasing function $h$. But for any bounded region $\mathcal{O}_{1}$ there exists a constant $\mu>0$ such that $\mathcal{O}_{1} \subset(x+(\lambda(x)-\mu) \mathcal{O})^{\prime}$ if $\lambda(x)$ is sufficiently large, hence $\mathfrak{U}\left(\mathcal{O}_{1}\right) \subset \mathfrak{A}(x+(\lambda(x)-\mu) \mathcal{O})^{\prime}=\rho(\mathfrak{H}(x+(\lambda(x)-\mu) \mathcal{O}))^{\prime}$. Thus for any $A \in \mathfrak{U}\left(\mathcal{O}_{1}\right)$ $\left|\partial_{\mu} \varphi_{x}(A)\right|$ is rapidly decreasing, so $\varphi=\lim _{x} \varphi_{x}$ exists and is a locally normal state on $\mathfrak{U}$.

By the GNS-construction applied to the state $\varphi$ one obtains a Hilbert space $\mathscr{H}_{\varphi}$, a cyclic vector $\Omega_{\varphi}$ and a representation $\pi_{\varphi}$ such that

$$
\left(\Omega_{\varphi}, \pi_{\varphi}(A) \Omega_{\varphi}\right)=\varphi(A), A \in \mathfrak{A} .
$$

As in [17] one can establish now the following facts:

i) $\pi_{\varphi}$ is localizable in spacelike cones, so there exists a $\bar{\rho} \in \Delta_{\mathscr{S}_{0}}$ with $\bar{\rho} \simeq \pi_{\varphi}$. ii) $\bar{\rho} \rho$ contains a subrepresentation which is equivalent to the vacuum representation. iii) There exists an isometric intertwiner $R$ from $\imath$ to $\bar{\rho} \rho$ such that $(R \Omega, \bar{\rho}(A) R \Omega)=$ $\varphi(A)$, and

$$
\phi(B)=R^{*} \bar{\rho}^{\mathscr{S}_{a}}(B) R, B \in \mathfrak{B}^{\mathscr{S}_{a}}
$$

defines a left-inverse $\phi$ of $\rho^{\mathscr{S}_{a}}$. iv) $\bar{\rho}$ is translation covariant, the translations $\mathscr{U}_{\rho}$ being defined by

$$
U_{\bar{\rho}}(x) \cdot \bar{\rho}(A) R \Omega=\bar{\rho}\left(\alpha_{x}(A)\right) \bar{\rho}^{\mathscr{S}_{a}}\left(U_{0}(x) U_{\rho}(-x)\right) R \Omega, A \in \mathfrak{A} .
$$

$\left(U_{0}(x)\right.$ are the translations in the vacuum representation and $U_{0}(x) U_{\rho}(-x) \in \mathfrak{B}^{\mathscr{S}_{a}}$ for any $x$.) v) $\mathscr{U}_{\bar{\rho}}$ fulfils the spectrum condition, sp $\mathscr{U}_{\bar{\rho}} \subset \bar{V}_{+}$.

So $\bar{\rho}$ is a reasonable conjugate representation. It remains to show that $\phi\left(\varepsilon_{\rho}\right) \neq 0$. This is achieved by an analysis of the two-point function of generalized fields connecting the vacuum and the particle sector.

Consider two mutually spacelike cones $\mathscr{S}_{1}, \mathscr{S}_{2} \subset \mathscr{S}_{0}^{\prime} \cap \mathscr{S}_{a}^{\prime}$ and let $\left\{\rho_{i}, B_{i}\right\} \in$ $\mathfrak{F}\left(\mathscr{S}_{i}\right), i=1,2$. Then, by a straightforward computation one finds that

$$
B_{1} B_{2}^{*}=\rho^{\mathscr{S}_{a}}\left(B_{2}^{*}\right) \varepsilon_{\rho} \rho^{\mathscr{S}_{a}}\left(B_{1}\right)
$$


and consequently

$$
\phi\left(B_{1} B_{2}^{*}\right)=B_{2}^{*} \phi\left(\varepsilon_{\rho}\right) B_{1} .
$$

Hence if $\phi\left(\varepsilon_{\rho}\right)=0$ it follows from (iii) that

$$
\left(\bar{\rho}^{\mathscr{S}_{a}}\left(B_{1}^{*}\right) R \Omega, \bar{\rho}^{\mathscr{S}_{a}}\left(B_{2}^{*}\right) R \Omega\right)=0 .
$$

Now if $x$ is such that $x+\mathscr{S}_{2} \subset \mathscr{S}_{2}$, then $\left\{\rho, U_{\rho}(x) B_{2} U_{0}(-x)\right\} \in \mathfrak{F}\left(\mathscr{S}_{2}+x\right) \subset$ $\mathfrak{F}\left(\mathscr{S}_{2}\right)$, and using (iv) one gets

$$
\left(\bar{\rho}^{\mathscr{S}_{a}}\left(B_{1}^{*}\right) R \Omega, U_{\bar{\rho}}(x) \bar{\rho}^{\mathscr{S}_{a}}\left(B_{2}^{*}\right) R \Omega\right)=0
$$

for the restricted set of $x$. But since according to (v) $\operatorname{sp} \mathscr{U}_{\bar{\rho}} \subset \bar{V}_{+}$this result holds for all $x$ by the edge-of-the-wedge theorem. Now if $V_{i}$ are intertwiners from $\rho$ to $\rho_{i} \in \Delta_{\mathscr{S}_{i}}$ and if $A \in \mathfrak{U}$ is any local observable, then $\left\{\rho, V_{i}^{-1} A\right\} \in \mathfrak{F}\left(\mathscr{S}_{i}+y\right)$ for some $y$, so from the above result

$$
\left(\bar{\rho}^{\mathscr{S}_{a}}\left(V_{1}\right) R \Omega, \bar{\rho}(A) \bar{\rho}^{\mathscr{S}_{a}}\left(V_{2}\right) R \Omega\right)=0 \quad \text { for } \quad A \in \mathfrak{U} .
$$

Yet this is contradicting the facts that $R \Omega$ is cyclic for $\bar{\rho}(\mathfrak{A})$ and $\bar{\rho}^{\mathscr{S}_{a}}$ normal on $\mathfrak{U}^{c}\left(\mathscr{S}_{a}+x\right)^{-}$for any $x$. Therefore $\phi\left(\varepsilon_{\rho}\right) \neq 0$ for the left-inverse defined in (iii).

Taking into account the previous discussion of reducible representations we get

Theorem 6.5. Let $\pi$ be a (factorial) single particle representation. Then $\pi$ is equivalent to a multiple of an irreducible single particle representation with finite statistics. (Note that in the derivation of this result the assumption of duality in the vacuum representation was used.)

In view of this result it is natural to define the class $(P) \subset(S)$ of particle representations which are generated from the irreducible single particle representations by composition, performing direct sums, taking subrepresentations and conjugates. We shall restrict our attention in the remainder of this article to $(P)$ and show that it describes all states of interest in particle physics.

In conclusion we remark that the results of this section can be used to equip the field bundles $\mathfrak{F}^{\mathscr{S}_{a}}$ with the notion of an adjoint. If $\rho$ is irreducible with finite statistics, then according to Theorem 6.3 there exists a conjugate representation $\bar{\rho}$ such that $\bar{\rho} \rho$ contains a subrepresentation which is equivalent to the vacuum representation. Now let $\boldsymbol{B}=\{\rho, B\} \in \mathfrak{F}^{\mathscr{S}_{a}}$ and let $R$ be an isometric intertwiner from $l$ to $\bar{\rho} \rho$. Then an adjoint $\boldsymbol{B}^{\dagger}$ of $\boldsymbol{B}$ can be defined by

$$
\boldsymbol{B}^{\dagger}=\left\{\bar{\rho}, \bar{\rho}^{\mathscr{S}_{a}}\left(B^{*}\right) R\right\}
$$

Clearly, the adjoint of $\boldsymbol{B}$ is not unique, and it requires some lengthy calculations in order to verify that $\boldsymbol{B}^{\dagger}$ has all desired properties. Fortunately, the results are the same as in [DHR II], so we can omit the details. If $\boldsymbol{\Phi}_{1}=\left\{\rho_{1}, \Phi_{1}\right\}$ and $\boldsymbol{\Phi}_{2}=$ $\left\{\rho_{1} \rho, \Phi_{2}\right\}$, a useful relation is

$$
\left(\boldsymbol{B} \Phi_{1}, \Phi_{2}\right)=\left(\boldsymbol{T} \cdot \boldsymbol{\Phi}_{1}, \boldsymbol{B}^{\dagger} \boldsymbol{\Phi}_{2}\right)
$$

where

$$
\boldsymbol{T}=\left(\rho_{1} \rho \bar{\rho}|T| \rho_{1}\right)
$$


and $T$ is explicitly given by

$$
T=\lambda_{\rho}^{-1} \cdot \rho_{1}^{\mathscr{S}_{a}}(\varepsilon(\bar{\rho}, \rho) R)
$$

(A direct proof of this statement can be given, using the calculations in the proof of Proposition 6.4.) The remaining statements are more easily verified. Since $\bar{\rho}$ is isometric one has

$$
\left\|\boldsymbol{B}^{\dagger}\right\| \leqq\|\boldsymbol{B}\|
$$

Finally, it follows from the duality of observables that the adjoints $\boldsymbol{B}^{\dagger}$ have the same localization properties as $\boldsymbol{B}$,

$$
\boldsymbol{B} \in \mathfrak{F}(\mathscr{S}) \Leftrightarrow \boldsymbol{B}^{\dagger} \in \mathfrak{F}(\mathscr{S}) .
$$

\section{Energy and Momentum in Composite Sectors}

The starting point of our investigations were spectral properties of the energymomentum operators in massive single particle representations, and we want to study now the energy-momentum spectrum in arbitrary representations of the class $(P)$. Again the arguments are analogous to the corresponding ones in [DHR II], but there is one little difference: in [DHR II] the absence of nontrivial finite dimensional unitary representations of the Poincaré-group was used in the analysis of the translation operators in the composite sectors. Since we did not require Lorentz-covariance of single particle representations we cannot exploit this fact here. Instead we will use the information on the spectrum in single particle representations for the proof that the energy-momentum spectrum has the physically expected structure in all particle representations in $(P)$.

We proceed as follows: starting from single particle representations we define for any $\rho \in(P)$ a representation $x \rightarrow U_{\rho}(x)$ of the translations. If $\rho$ is reducible, there are several possible candidates for $\mathscr{U}_{\rho}$, and we will take our choice in such a way that the operators $U_{\rho}(x)$ can be used later for the definition of translations on the field bundle. We will see that with this choice the spectrum condition holds, sp $\mathscr{U}_{\rho} \subset \bar{V}_{+}$, that the lower boundary of $\operatorname{sp} \mathscr{U}_{\rho}$ is Lorentz-invariant and that $U_{\rho}(x) \in \rho(\mathfrak{H})^{-}$.

Now let $\rho \in \Delta_{\mathscr{S}_{0}}$ be an irreducible single particle representation. We denote by $x \rightarrow U_{\rho}(x)$ the strongly continuous unitary representation of the translations, implementing the automorphisms $\alpha_{x}$ in the representation $\rho . \operatorname{sp} \mathscr{U}_{\rho}$ contains an isolated hyperboloid $H_{m}$,

$$
H_{m} \subset \operatorname{sp} \mathscr{U}_{\rho} \subset H_{m} \cup\left\{p: p^{2} \geqq M^{2}, p_{0}>0\right\},
$$

where $M>m>0$ (cf. Sect. 2).

It has been shown in [17] that any conjugate representation $\bar{\rho}$ of $\rho$ is again an irreducible single particle representation. We sketch here a slightly modified proof of this fact in order to display, where the specific form of $\operatorname{sp} \mathscr{U}_{\rho}$ is used: since $\rho$ has finite statistics, there exists an irreducible conjugate representation $\bar{\rho}$ such that $\bar{\rho} \rho$ contains a subrepresentation which is equivalent to $l$, i.e.

$$
\bar{\rho} \rho(A) R=R A, \quad A \in \mathfrak{A}
$$


for some isometry $R$. The unique left-inverse of $\rho^{\mathscr{S}_{a}}, \mathscr{S}_{a} \subset \mathscr{S}_{0}^{\prime}$, which is weakly continuous on $\mathfrak{U}^{c}\left(\mathscr{S}_{a}+x\right)^{-}$for all $x$ is therefore given by $\phi^{\mathscr{S}_{a}}(\cdot)=R^{*} \bar{\rho}^{\mathscr{S}_{a}}(\cdot) R$. Using the localization properties of $\rho$ we find that

$$
U_{0}(x) U_{\rho}(-x) \in \mathfrak{U}\left(\mathscr{S}_{a}+x\right)^{\prime}=\mathfrak{U}^{c}\left(\mathscr{S}_{a}+x\right)^{-},
$$

so the translations $U_{\rho}(x)$ induce automorphisms $\beta_{x}$ of $\mathfrak{B}^{\mathscr{S}_{a}}$. Consequently, $\alpha_{-x}{ }^{\circ}$ $\phi^{\mathscr{S}_{a} \circ} \beta_{x}$ is also a left-inverse of $\rho^{\mathscr{S}_{a}}$ with the same continuity properties as $\phi^{\mathscr{S}_{a}}$, hence it is equal to $\phi^{\mathscr{S}_{a}}$. So the state $\omega_{0} \phi^{\mathscr{S}_{a}}$ is invariant under $\beta_{x}$, and we can define translation operators $U_{\bar{\rho} \rho}(x)$ in the representation $\bar{\rho} \rho$ by

$$
U_{\bar{\rho} \rho}(x) \bar{\rho}^{\mathscr{S}_{a}}(B) R \Omega=\bar{\rho}^{\mathscr{S}_{a}}\left(\beta_{x}(B)\right) R \Omega, \quad B \in \mathfrak{B}^{\mathscr{S}_{a}} .
$$

We remark for later reference that $\mathscr{U}_{\bar{\rho} \rho}$ is equivalent to $\mathscr{U}_{0}$ on the range of $R$,

$$
U_{\bar{\rho} \rho}(x) R=R U_{0}(x) .
$$

Translations in the conjugate representation $\bar{\rho}$ can now be defined by

$$
U_{\bar{\rho}}(x)=\bar{\rho}^{\mathscr{S}_{a}}\left(U_{0}(x) U_{\rho}(-x)\right) U_{\bar{\rho} \rho}(x),
$$

and they are continuous because $\bar{\rho}^{\mathscr{S}_{a}}$ is normal on $\mathfrak{A}^{c}\left(\mathscr{S}_{a}+x\right)^{-}$for all $x$. One can show then as in [DHR II] (see also Lemma 7.6) that $\operatorname{sp} \mathscr{U}_{\bar{\rho}}+\operatorname{sp} \mathscr{U}_{\rho} \subset \operatorname{sp} \mathscr{U}_{0}$ and therefore sp $\mathscr{U}_{\bar{\rho}} \subset \bar{V}_{+}$. Since $\bar{\rho}$ is irreducible the arguments of Sect. 2 can be applied proving that the set $\left(\operatorname{sp} \mathscr{U}_{\bar{\rho}}+p\right)$ has a Lorentz-invariant lower boundary for some $p \in \mathbb{R}^{4}$. However, we will need that $p=0$.

This additional piece of information is obtained by applying the methods of the Jost-Lehmann-Dyson representation to the two-point function of generalized fields. If $\mathscr{S}_{1}^{\prime}, \mathscr{S}_{2} \subset \mathscr{S}_{0}^{\prime} \cap \mathscr{S}_{a}^{\prime}$ are two mutually spacelike cones and if there is a cone $\mathscr{S}_{3} \supset\left(\mathscr{S}_{1}-\mathscr{S}_{2}\right)$, then it follows from the general relation (6.28) that

$$
\left(\bar{\rho}^{\mathscr{S}_{a}}\left(B_{1}^{*}\right) R \Omega, U_{\bar{\rho}}(x) \bar{\rho}^{\mathscr{S}_{a}}\left(B_{2}^{*}\right) R \Omega\right)=\lambda_{\rho}\left(B_{2} \Omega, U_{\rho}(-x) B_{1} \Omega\right)
$$

if $\left\{\rho, B_{i}\right\} \in \mathfrak{F}\left(\mathscr{S}_{i}\right), i=1,2$ and $x \in \mathscr{S}_{3}^{\prime}$. Normally one exploits in the Jost-LehmannDyson representation the fact that a relation of the type (7.7) holds for $x$ in the causal complement of some bounded region. However, the region $\mathscr{S}_{3}^{\prime}$ is still big enough (it contains in particular a wedge-region) to draw as in [26] and [DHR II] the conclusion that the spectra of the mass operators $P_{\rho}^{2}$ and $P_{\bar{\rho}}^{2}$ coincide. If we would only know that $\operatorname{sp} \mathscr{U}_{\rho} \subset \bar{V}_{+}$this would merely restrict $p$ to a lightlike vector, $p^{2}=0$. But from the more specific information (7.1) it follows that $p=0$. So the particular strueture of the energy-momentum spectrum in particle representations is used at the very end of this analysis. It is then clear that relation (7.1) holds also for sp $\mathscr{U}_{\bar{\rho}}$ with the same mass-values $m$ and $M$. We summarize the results of this discussion in the following theorem [17].

Theorem 7.1. The conjugate representation $\bar{\rho} \in \Delta_{\mathscr{S}_{0}}$ of an irreducible single particle representation $\rho \in \Delta_{\mathscr{S}_{0}}$ is an irreducible single particle representation with translations $\mathscr{U}_{\bar{\rho}}$ given by relation (7.6). Moreover,

$$
\left\{p^{2}: p \in \operatorname{sp} \mathscr{U}_{\rho}\right\}^{-}=\left\{p^{2}: p \in \operatorname{sp} \mathscr{U}_{\bar{\rho}}\right\}^{-},
$$

i.e. the mass-spectra in the representations $\rho$ and $\bar{\rho}$ coincide. 
Recalling, that the statistics parameters of $\rho$ and $\bar{\rho}$ are equal, this theorem reproduces in our general setting the familiar fact that each particle has an antiparticle with the same mass and statistics.

Since one can use the elementary conjugates of single particle representations to build up conjugates of arbitrary representations in $(P)$ by composition, taking direct sums and subrepresentations it is sufficient to study the structure of the translations in representations arising from single particle representations by these operations.

Now let $\rho_{1}, \rho_{2} \in \Delta_{\mathscr{S}_{0}}$ be two representations in which continuous unitary representations $\mathscr{U}_{\rho_{1}}$ and $\mathscr{U}_{\rho_{2}}$ of the translations are defined. Then $\rho_{1} \rho_{2}$ is also a covariant representation with translation operators given by

$$
U_{\rho_{1} \rho_{2}}(x)=\rho_{1}^{\mathscr{S} a}\left(U_{\rho_{2}}(x) U_{0}(-x)\right) U_{\rho_{1}}(x) .
$$

(Note that if $\rho_{1}=\bar{\rho}$ and $\rho_{2}=\rho$ this definition is consistent with relation (7.6).) It can easily be checked that the composition law (7.8) for representations of the translation group is associative. We will see that with this definition the translations in the composed representations in $(\mathrm{P})$ have all the desired properties.

Given irreducible single particle representations $\rho_{i} \in \Delta_{\mathscr{S}_{0}}, i=1, \ldots n$ with translations $\mathscr{U}_{\rho_{i}}$ we can construct with the help of (7.8) translations $\mathscr{U}_{\rho_{1} \ldots \rho_{n}}$ in the representation $\rho_{1} \cdots \rho_{n}$. For the analysis of the spectral properties of $\mathscr{U}_{\rho_{1} \ldots \rho_{n}}$ the following lemma is needed.

Lemma 7.2. Let $\rho \in \Delta_{\mathscr{S}_{0}}$ be an irreducible representation with finite statistics in which translations $U_{\rho}(x)$ are defined, and let $\beta_{x}$ be the automorphism of $\mathfrak{B}^{\mathscr{S} a}, \mathscr{S}_{a} \subset \mathscr{S}_{0}^{\prime}$ induced by $U_{\rho}(x)$. If $x$ is a sequence of translations which tends to spacelike infinity in some suitable subcone of $-\mathscr{S}_{a}$, then

$$
w-\lim _{x} \beta_{x}(B)=\omega_{0}{ }^{\circ} \phi^{\mathscr{S}_{a}}(B) \cdot 1, \quad B \in \mathfrak{B}^{\mathscr{S}_{a}},
$$

where $\phi^{\mathscr{S}_{a}}$ is the left-inverse of $\rho^{\mathscr{S}_{a}}$ distinguished by Proposition 6.2.

Proof. For suitable sequences $x \in-\mathscr{S}_{a}$ the norm of the commutator $\left[\beta_{x}(B), \rho(A)\right]$ tends to zero for all $A \in \mathfrak{U}$ and $B \in \mathfrak{B}^{\mathscr{S}_{a}}$ because of locality. So taking into account that $\rho$ is irreducible, it follows that each weak limit point of $\beta_{x}$ is of the form $\omega(\cdot) \cdot 1$, where $\omega$ is some state on $\mathfrak{B}^{\mathscr{S}}$. Moreover, since

$$
w-\lim _{x} \alpha_{x}(B)=\omega_{0}(B) \cdot 1, \quad B \in \mathfrak{B}^{\mathscr{S}_{a}}
$$

and since $\rho^{\mathscr{S}_{a}}$ is weakly continuous on $\mathfrak{A}^{c}\left(\mathscr{S}_{a}+y\right)^{-}$for all $y$ one finds that

$$
\omega\left(\rho^{\mathscr{S}_{a}}(B)\right)=\omega_{0}(B), \quad B \in \mathfrak{B}^{\mathscr{S}_{a}},
$$

So if $\pi_{\omega}$ denotes the representation of $\mathfrak{B}^{\mathscr{S}_{a}}$ which is induced by $\omega$ it is clear that $\pi_{\omega}{ }^{\circ} \rho^{\mathscr{S}_{a}}$ contains a subrepresentation which is equivalent to the vacuum representation, hence $\omega=\omega_{0}{ }^{\circ} \phi$ for some left-inverse $\phi$. The uniqueness result on leftinverses in Sect.6 then implies that all weak limit points of $\beta_{x}$ coincide, proving the statement. QED.

Using this result we can analyse now the energy-momentum spectrum in translational covariant subrepresentations of $\rho_{1} \cdots \rho_{n}$. (Actually any subrepresentation of $\rho_{1} \cdots \rho_{n}$ is covariant as we shall see later.) If $E \in \rho_{1} \cdots \rho_{n}(\mathfrak{H})^{\prime}$ is a projection 
which commutes with $U_{\rho_{1} \ldots \rho_{n}}(x)$ we define translations $\mathscr{U}_{E}$ on $E \mathscr{H}_{0}$ by

$$
U_{E}(x)=U_{\rho_{1} \ldots \rho_{n}}(x) E
$$

and find as in [DHR II, Thm. 5.2]:

Lemma 7.3. $\operatorname{sp} \mathscr{U}_{E} \supset \operatorname{sp} \mathscr{U}_{\rho_{1}}+\ldots+\operatorname{sp} \mathscr{U}_{\rho_{n}}$.

Proof. We sketch the argument for $n=2$. Let $B_{1}, B_{2} \in \mathfrak{B}^{\mathscr{S}_{a}}$, then from relation (7.8)

$$
U_{E}(x) \cdot E \rho_{1}^{\mathscr{S}_{a}}\left(B_{2}\right) B_{1} \Omega=E \rho_{1}^{\mathscr{S}_{a}}\left(U_{\rho_{2}}(x) B_{2} U_{0}(-x)\right) U_{\rho_{1}}(x) B_{1} \Omega .
$$

Thus if we set

$$
B_{i}=\int d^{4} x f_{i}(x) U_{\rho_{i}}(x) A_{i} U_{0}(-x), \quad i=1,2,
$$

where $A_{i} \in \mathfrak{A}$ and $f_{i}$ are testfunctions, it follows that the energy-momentum support (with respect to $\mathscr{U}_{E}$ ) of the vectors

$$
\Psi_{y_{1} y_{2}}=E \rho_{1}^{\mathscr{S}_{a}}\left(U_{\rho_{2}}\left(y_{2}\right) B_{2} U_{0}\left(-y_{2}\right)\right) U_{\rho_{1}}\left(y_{1}\right) B_{1} \Omega
$$

is contained in supp $\tilde{f}_{1}+\operatorname{supp} \tilde{f}_{2}$. Hence if we can prove that this vector is different from zero for some $y_{1}, y_{2}$ whenever $\left\|B_{1} \Omega\right\| \cdot\left\|B_{2} \Omega\right\| \neq 0$, the assertion follows.

We have seen in the previous lemma that the automorphisms $\beta_{y}^{\rho_{1}} i=1,2$ converge weakly to $\omega_{0}{ }^{\circ} \phi_{\rho_{t}}^{\mathscr{S}_{a}}(\cdot) \cdot 1$ if $y$ tends to spacelike infinity in the way described. Thus we can proceed as in [DHR II, Thm. 5.2] and send first $y_{1}$ to spacelike infinity and then $y_{2}$. The result is

$$
\lim _{y_{2}} \lim _{y_{1}}\left\|\Psi_{y_{1} y_{2}}\right\|^{2}=\left\|B_{1} \Omega\right\|^{2} \cdot\left\|B_{2} \Omega\right\|^{2} \cdot\left(\Omega, \phi_{\rho_{2}}^{\mathscr{S}_{a}} \phi_{\rho_{1}}^{\mathscr{S}_{a}}(E) \Omega\right),
$$

and it remains to show that the vacuum expectation value of $\phi_{\rho_{2}}^{\mathscr{S}_{a}} \phi_{\rho_{1}}^{\mathscr{S}_{a}}(E)$ is different from zero. But this is obvious since $\phi_{\rho_{2}}^{\mathscr{S}_{a}} \phi_{\rho_{1}}^{\mathscr{S}_{a}}(E)$ is a multiple of the identity which cannot be zero because the left-inverses $\phi_{\rho_{l}}^{\mathscr{S}_{a}}$ act faithfully on $\mathfrak{B}^{\mathscr{S}_{a}}$. QED.

This result shows in particular that $\mathrm{sp} \mathscr{U}_{E}$ contains the sum of the single particle mass shells $H_{m_{l}}$ of the single particle representations $\rho_{i}$,

$$
\operatorname{sp} \mathscr{U}_{E} \supset\left\{p: p^{2} \geqq\left(\sum_{1}^{n} m_{i}\right)^{2}, p_{0}>0\right\} .
$$

Therefore, any light cone containing sp $\mathscr{U}_{E}$ contains also $V_{+}$. In the next step we will show that sp $\mathscr{U}_{\rho_{1}} \cdots \rho_{n}$ (and therefore also sp $\mathscr{U}_{E}$ ) is contained in $\bar{V}_{+}$.

Lemma 7.4. $\operatorname{sp} \mathscr{U}_{\rho_{1}} \cdots \rho_{n} \subset \bar{V}_{+}$.

Proof. Again we give the argument only for $n=2$. Let $R=\bar{\rho}_{2}^{\mathscr{S}_{a}}\left(R_{1}\right) R_{2}$, where $R_{i}, i=1,2$ are isometric intertwiners from $\imath$ to $\bar{\rho}_{i} \rho_{i}$. Then $R$ is an isometric intertwiner from $l$ to $\bar{\rho}_{2} \bar{\rho}_{1} \rho_{1} \rho_{2}$. Defining the translations $\mathscr{U}_{\bar{\rho}_{2} \bar{\rho}_{1} \rho_{1} \rho_{2}}$ in the representation $\bar{\rho}_{2} \bar{\rho}_{1} \rho_{1} \rho_{2}$ by the composition law (7.8), one arrives by repeated use of the relations (7.5), (7.6) and (7.8) as well as of the fact that $R_{1}$ is an intertwiner from $l$ to $\bar{\rho}_{1} \rho_{1}$ at

$$
U_{\bar{\rho}_{2} \bar{\rho}_{1} \rho_{1} \rho_{2}}(x) R=R U_{0}(x),
$$

which is the analogue of relation (7.5). So the projection $F=R R^{*}$ commutes with 
$U_{\bar{\rho}_{2} \bar{\rho}_{1} \rho_{1} \rho_{2}}(x)$ and we can define translations on $F \mathscr{H}_{0}$ by

$$
U_{F}(x)=U_{\bar{\rho}_{2} \bar{\rho}_{1} \rho_{1} \rho_{2}}(x) F .
$$

We want to show that $\operatorname{sp} \mathscr{U}_{\bar{\rho}_{1}}+\operatorname{sp} \mathscr{U}_{\bar{\rho}_{2}}+\operatorname{sp} \mathscr{U}_{\rho_{1} \rho_{2}} \subset \operatorname{sp} \mathscr{U}_{F}$; the statement then follows since $\operatorname{sp} \mathscr{U}_{F} \subset \operatorname{sp} \mathscr{U}_{0}$ by the above relation. As in the proof of Lemma 7.2 it is sufficient to verify that the vector

$$
\Psi_{y_{1} y_{2} y_{3}}=F \cdot\left(\bar{\rho}_{2} \bar{\rho}_{1}\right)^{\mathscr{S}_{a}}\left(U_{\rho_{1} \rho_{2}}\left(y_{3}\right) B_{3} U_{0}\left(-y_{3}\right)\right) \bar{\rho}_{2}^{\mathscr{S}_{a}}\left(U_{\bar{\rho}_{1}}\left(y_{2}\right) B_{2} U_{0}\left(-y_{2}\right)\right) U_{\bar{\rho}_{2}}\left(y_{1}\right) B_{1} \Omega
$$

is different from zero for some $y_{1}, y_{2}, y_{3}$ whenever $\left\|B_{1} \Omega\right\| \cdot\left\|B_{2} \Omega\right\| \cdot\left\|B_{3} \Omega\right\| \neq 0$. Now since $\bar{\rho}_{1}, \bar{\rho}_{2}$ are irreducible representations we can let $y_{1}$ and $y_{2}$ go to spacelike infinity, giving

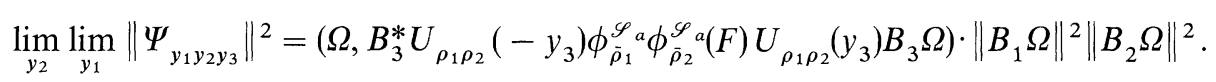

At this point we cannot perform the limit with respect to $y_{3}$ by applying Lemma 8.2 , because $\rho_{1} \rho_{2}$ need not be an irreducible representation. But for the particular $F$ which is of interest here we find that

$$
\phi_{\bar{\rho}_{1}}^{\mathscr{S}_{a}} \phi_{\bar{\rho}_{2}}^{\mathscr{S}_{a}}(F)=\phi_{\bar{\rho}_{1}}^{\mathscr{S}_{a}}\left(R_{1} R_{1}^{*}\right) \phi_{\bar{\rho}_{2}}^{\mathscr{S}_{a}}\left(R_{2} R_{2}^{*}\right)=\lambda \cdot 1 \neq 0
$$

because each $\phi_{\bar{\rho}_{1}}^{\mathscr{S}}\left(R_{i} R_{i}^{*}\right)$ is a non-zero multiple of the identity. So

$$
\lim _{y_{2}} \lim _{y_{1}}\left\|\Psi_{y_{1} y_{2} y_{3}}\right\|^{2}=\lambda\left\|B_{1} \Omega\right\|^{2}\left\|B_{2} \Omega\right\|^{2}\left\|B_{3} \Omega\right\|^{2}
$$

and the assertion follows. QED.

Next we will prove that the translations $U_{\rho_{1} \ldots \rho_{n}}(x)$ defined by the composition law (7.8) are elements of $\rho_{1} \ldots \rho_{n}(\mathfrak{H})^{-}$. We will establish this fact by showing that each projection $E \in \rho_{1} \cdots \rho_{n}(\mathfrak{Y})^{\prime}$ commutes with $U_{\rho_{1} \ldots \rho_{n}}(x)$. As in the corresponding argument in [DHR II] it is crucial that $\rho_{1} \cdots \rho_{n}$ is a finite direct sum of irreducible representations because the particle representations $\rho_{i}$ have finite statistics (cf. the discussion in Sect.6 and [DHR I]).

Lemma 7.5. $U_{\rho_{1} \ldots \rho_{n}}(x) \in \rho_{1} \ldots \rho_{n}(\mathfrak{A})^{-}$

Proof. Since $\operatorname{sp} \mathscr{U}_{\rho_{1} \ldots \rho_{n}} \subset \bar{V}_{+}$each central projection $E$ in $\rho_{1} \ldots \rho_{n}(\mathfrak{A})^{\prime}$ commutes with $U_{\rho_{1} \ldots \rho_{n}}(x)[19,27]$. So let $E$ be a minimal central projection and let $\mathscr{U}_{E}$ be the subrepresentation of the translations $\mathscr{U}_{\rho_{1} \ldots \rho_{n}}$ on $E \mathscr{H}_{0}$. According to the result [19] of Borchers quoted in Sect.2 one can find translations $\hat{U}_{E}(x) \in \rho_{1} \ldots \rho_{n}(\mathfrak{U})^{-} E$ inducing the same automorphism on $\rho_{1} \ldots \rho_{n}(\mathfrak{A})^{-} \cdot E$ as $U_{E}(x)$. Moreover, since $\rho_{1} \ldots \rho_{n}(\mathfrak{U})^{-} \cdot E$ is a factor one can choose $\hat{\mathscr{U}}_{E}$ such that the lower boundary of sp $\hat{\mathscr{U}}_{E}$ is Lorentz-invariant (Proposition 2.1). Setting $\breve{U}_{E}(x)=U_{E}(x) \hat{U}_{E}(-x)$ it is clear that $\breve{U}_{E}(x) \in \rho_{1} \ldots \rho_{n}(\mathfrak{U})^{\prime} E$, and since $\rho_{1} \ldots \rho_{n}(\mathfrak{U})^{\prime} E$ is a finite, discrete von Neumann algebra it follows by spectral decomposition that

$$
\check{U}_{E}(x)=\sum_{k=1}^{m} e^{i p_{k} x} \cdot E_{k},
$$

where $E_{1}, \ldots E_{m}$ are mutually orthogonal projections in $\rho_{1} \ldots \rho_{n}(\mathfrak{A})^{\prime} \cdot E$. Now the spectrum of $\hat{\mathscr{U}}_{E}$ is the same on all subspaces $E_{k} \mathscr{H}_{0}, k=1, \ldots m$, because 
$\rho_{1} \ldots \rho_{n}(\mathfrak{X})^{-} \cdot E$ is a factor. Moreover,

$$
U_{\rho_{1} \ldots \rho_{n}}(x) E_{k}=E_{k} U_{\rho_{1} \ldots \rho_{n}}(x), k=1, \ldots m,
$$

so applying Lemma 7.3 and 7.4 to $U_{E_{k}}(x)=U_{\rho_{1} \ldots \rho_{n}}(x) E_{k}$ one arrives at

$$
H_{m_{1}}+\ldots+H_{m_{n}} \subset \operatorname{sp} \hat{\mathscr{U}}_{E}+p_{k} \subset \bar{V}_{+}, k=1, \ldots m,
$$

which is only compatible with the Lorentz-invariant boundary of $\mathrm{sp} \hat{\mathscr{U}}_{E}$ if $p_{k}=0, k=1, \ldots m$. So $\breve{\mathscr{U}}_{E}$ is the trivial representation, and the assertion follows.

QED.

By repeated application of the relations (7.6), (7.8) and (7.9) one can construct now for each $\rho \in(P)$ a representation $\mathscr{U}_{\rho}$ of the translations such that $\operatorname{sp} \mathscr{U}_{\rho} \subset$ $\bar{V}_{+}, U_{\rho}(x) \in \rho(\mathfrak{U})^{-}$and the lower boundary of $\operatorname{sp} \mathscr{U}_{\rho}$ is Lorentz-invariant in each subrepresentation of $\rho$; these properties characterize $\mathcal{U}_{\rho}$ uniquely.

Finally, we will show that $\operatorname{sp} \mathscr{U}_{\rho}$ has a lower mass gap. This is needed in the calculations of scalar products of scattering states in the next section.

Lemma 7.6. Let $\rho \in(P)$. Then

$$
\operatorname{sp} \mathscr{U}_{\rho} \subset\{0\} \cup\left\{p: p^{2} \geqq \frac{\mu^{2}}{4}, p_{0}>0\right\},
$$

where $\mu$ is the mass-gap in the vacuum representation. $0 \in \mathrm{sp} \mathscr{U}_{\rho}$ if and only if $\rho$ contains a subrepresentation which is equivalent to the vacuum representation.

Proof. If $\rho$ is irreducible it follows from the argument given in Lemma 7.3 that $\operatorname{sp} \mathscr{U}_{\bar{\rho}}+\operatorname{sp} \mathscr{U}_{\rho} \subset \operatorname{sp} \mathscr{U}_{0}$. On the other hand relation (7.7) holds for the expectation values of $U_{\rho}(x)$ and $U_{\bar{\rho}}(x)$, and applying the methods of the Jost-Lehmann-Dyson representation one finds that the mass spectra of $\mathscr{U}_{\rho}$ and $\mathscr{U}_{\bar{\rho}}$ coincide. These two facts establish the first part of the statement for irreducible $\rho$. But then it holds for arbitrary $\rho \in(P)$ because any such $\rho$ can be decomposed into a finite direct sum of irreducible representations and $U_{\rho}(x) \in \rho(\mathfrak{U})^{-}$.

Now if $\rho$ is reducible and if there is a vector $\Phi$ which is invariant under $U_{\rho}(x)$, then for all $x \in \mathbb{R}^{4}$

$$
(\Phi, \rho(A) \Phi)=\left(\Phi, \rho\left(\alpha_{x}(A)\right) \Phi\right)=\omega_{0}(A) \cdot\|\Phi\|^{2}, A \in \mathfrak{U},
$$

where the second equality is obtained in the limit of large spacelike $x \in \mathscr{S}_{a}$. So $\rho$ contains a subrepresentation which is equivalent to the vacuum representation. Conversely, if $\rho(A) R=R A, A \in \mathfrak{U}$ for some isometric intertwiner $R$, then $U_{\rho}(x) R=$ $R U_{0}(x)$ because of the uniqueness of $\mathscr{U}_{\rho}$, hence $R \Omega$ is invariant under the action of $U_{\rho}(x)$. QED.

We summarize the information obtained in this section in

Theorem 7.7. Let $\rho$ be a particle representation, $\rho \in(P)$. Then there exists a unique continuous, unitary representation $\mathscr{U}_{\rho}$ of the translations such that

i) $U_{\rho}(x) \rho(A) U_{\rho}(x)^{-1}=\rho\left(\alpha_{x}(A)\right)$ for $A \in \mathfrak{A}$.

ii) $\operatorname{sp} \mathscr{U}_{\rho} \subset\{0\} \cup\left\{p: p^{2} \geqq \frac{\mu^{2}}{4}, p_{0}>0\right\}$ 
and $0 \in \mathrm{sp} \mathscr{U}_{\rho}$ iff $\rho$ contains a subrepresentation which is equivalent to the vacuum representation.

iii) $\operatorname{sp} \mathscr{U}_{\rho}$ has a Lorentz-invariant lower boundary in each subrepresentation of $\rho$.

iv) $U_{\rho}(x) \in \rho(\mathfrak{H})^{-}$for all $x$.

We are now in a position to define translations on the vector bundle $\mathscr{H}$ and on the field bundles $\mathfrak{F}^{\mathscr{S}_{a}}$. To this end we restrict the definition of these bundles to the subset of particle representations in $\Delta_{\mathscr{S}_{0}}$. Then translations $\boldsymbol{U}(x)$ on $\mathscr{H}$ can be defined by

$$
\boldsymbol{U}(x)\{\rho, \Psi\}=\left\{\rho, U_{\rho}(x) \Psi\right\} .
$$

$\boldsymbol{U}(x)$ commutes with the action of intertwiners, and it induces automorphisms $\alpha_{x}$ on the field bundles,

$$
\alpha_{x}\{\rho, B\}=\boldsymbol{U}(x)\{\rho, B\} \boldsymbol{U}(-x)=\left\{\rho, U_{\rho}(x) B U_{0}(-x)\right\}
$$

which respect the local structure,

$$
\alpha_{x}(\mathfrak{F}(\mathscr{S})) \subset \mathfrak{F}(\mathscr{S}+x)
$$

Moreover, there exists an adjoint of $\alpha_{x}(\boldsymbol{B})$ such that

$$
\alpha_{x}(\boldsymbol{B})^{\dagger}=\alpha_{x}\left(\boldsymbol{B}^{\dagger}\right)
$$

(see [DHR II, Prop. 4.5]). These results are used in the construction of scattering states in the subsequent section.

\section{Scattering Theory}

Let $\rho_{1}, \ldots \rho_{n} \in \Delta_{\mathscr{S}_{0}}$ be irreducible single particle representations. We will show now that there are states in the composed representation $\rho_{1} \cdots \rho_{n}$ which look like configurations of $n$ freely moving particles at asymptotic times. This, finally, justifies the interpretation of the representations $\rho \in(P)$ as particle representations.

We construct these states applying the basic ideas of the Haag-Ruelle scattering theory [13]. The procedure is well known in local field theory, and it has been shown in [DHR II] how to extend the construction to representations which are localizable in bounded regions. Now in the present general case the fields which connect the vacuum and single particle states need not be localized in bounded regions, the best we can say is that they are localized in spacelike cones. In order to exploit these weaker localization properties we must base our construction of scattering states on asymptotic particle configurations, where the momenta of all particles point into different directions. But this is sufficient since these states are dense in the set of all scattering states.

As usual, the construction of scattering states is performed in a fixed Lorentzsystem which can be characterized by a positive timelike vector $e$ (the time-direction), $e^{2}=1$ and $e_{0}>0$. Given $e$, a spacelike cone $\mathscr{S}$ and the sector of a single particle representation $\rho \in \Delta_{\mathscr{S}_{0}}$ we define subspaces of the testfunction space $\mathscr{S}\left(\mathbb{R}^{4}\right)$. 
Definition. $f \in \mathscr{L}_{\rho}(\mathscr{S}, e) \subset \mathscr{S}\left(\mathbb{R}^{4}\right)$ if

i) $\operatorname{supp} \tilde{f}$ is compact and $\operatorname{supp} \tilde{f} \cap \operatorname{sp} \mathscr{U}_{\rho} \subset H_{m}$.

ii) There is a neighbourhood $\mathcal{O}$ of the origin in $\mathbb{R}^{4}$ such that for each $p \in \operatorname{supp} \tilde{f}$

$$
\frac{p-(p e) e}{\varepsilon_{e}(p)}+\mathscr{O} \subset(\mathscr{S}-a),
$$

where $\varepsilon_{e}(p)=\left(m^{2}+(p e)^{2}-p^{2}\right)^{1 / 2}$ and $a$ is the apex of $\mathscr{S}$.

These testfunction spaces can be used to construct single particle states of mass $m$ which enter at asymptotic times $t$ with certainty the region $\mathscr{S}+t e$. Let $\boldsymbol{B}=\{\rho, B\} \in \mathfrak{F}(\mathscr{S})$ and let $f \in \mathscr{L}_{\rho}(\mathscr{S}, e)$, then we set for $t>0$

$$
C(t e)=(2 \pi)^{-2} \int d^{4} x \int d^{4} p e^{-i p x+i\left(p e-\varepsilon_{e}(p)\right) t} \tilde{f}(p) \alpha_{x}(\boldsymbol{B}) .
$$

It is easy to verify that $\boldsymbol{C}(t e) \boldsymbol{\Omega}$ is a single particle state vector (in the fiber $\mathscr{H}_{\rho}$ of $\mathscr{H}$ ) which does not depend on $t$ and $e$. Moreover, making use of the asymptotic behaviour of smooth solutions of the Klein-Gordon equation [13] one finds that the operator $C(t e)$ is essentially localized in the region $\mathscr{S}+t(e+s) \subset \mathscr{S}+t e$, where $s \in \mathscr{S}-a$ is a fixed vector and $a$ is the apex of $\mathscr{S}$. More precisely, there exists a sequence of generalized fields $C_{t} \in \mathfrak{F}(\mathscr{S}+t(e+s))$ such that

$$
\left\|\boldsymbol{C}(t e)-C_{t}\right\|<h(t),
$$

$h$ being a rapidly decreasing function. So the single particle states can be constructed with the help of creation operators which are almost localized in spacelike cones.

The construction of multi-particle states is now accomplished as in [DHR II]. Let $\rho_{1}, \ldots \rho_{n} \in \Delta_{\mathscr{S}_{0}}$ be irreducible single particle representations. Then one picks mutually spacelike separated cones $\mathscr{S}_{1}, \ldots \mathscr{S}_{n}$ such that $\mathscr{S}_{0}^{\prime} \cap \mathscr{S}_{1}^{\prime} \cap \ldots \cap \mathscr{S}_{n}^{\prime}$ still contains some auxiliary cone $\mathscr{S}_{a}$ (this is necessary for the application of the field bundle formalism). One constructs then, as explained, single particle creation operators $C_{i}(t e), i=1, \ldots n$ which are essentially localized in the cones $\mathscr{S}_{i}+t\left(e+s_{i}\right)$ and applies the commutation relations (5.10), giving

$$
\left\|\prod_{i=1}^{n} C_{p^{-1}(i)}(t e)-\varepsilon_{p}\left(\rho_{n}, \ldots \rho_{1}\right) \circ \prod_{i=1}^{n} C_{i}(t e)\right\|<h(t)
$$

for any permutation $p \in P^{(n)}$, Since $C_{i}(t e) \boldsymbol{\Omega}$ is independent of $t$ it follows that (in the fiber $\mathscr{H}_{\rho_{n} \ldots \rho_{1}}$ )

$$
\left\|\frac{d}{d t} \prod_{i=1}^{n} C_{i}(t e) \boldsymbol{\Omega}\right\|<h(t)
$$

so the limit

$$
s-\lim _{t \rightarrow \infty} C_{1}(t e) \cdots C_{n}(t e) \boldsymbol{\Omega}
$$

exists. We remark that one can replace everywhere in these expressions the operators $C_{i}(t e)$ by $C_{i}\left(t e_{i}\right)$, provided the vectors $e_{i}$ are sufficiently close to $e$. So as in [DHR II, Prop. 7.1] we arrive at 
Lemma 8.1. Let $\boldsymbol{C}_{i}(t e), i=1, \ldots n$ be single particle creation operators with localization properties specified above. Then the strong limit

$$
s-\lim _{t \rightarrow \infty} C_{1}(t e) \ldots C_{n}(t e) \boldsymbol{\Omega}=\Psi^{\text {out }}
$$

exists. If the localization cones $\mathscr{S}_{1}, \ldots \mathscr{S}_{n}$ and the single particle states $\boldsymbol{C}_{i}($ te $) \boldsymbol{\Omega}=$ $\boldsymbol{\Psi}_{i}, i=1, \ldots n$ are kept fixed, $\boldsymbol{\Psi}^{\mathrm{out}}$ does not depend on the choice of the operators $C_{i}(t e)$ and of the time-direction $e$.

In view of this result we can write

$$
\boldsymbol{\Psi}^{\text {out }}=\boldsymbol{\Psi}_{1} \stackrel{\text { out }}{\times} \ldots \stackrel{\text { out }}{\times} \boldsymbol{\Psi}_{n},
$$

and it follows immediately from the above discussion that $\boldsymbol{\Psi}^{\text {out }}$ has the familiar behaviour under translations and permutations:

$$
\begin{aligned}
& \boldsymbol{U}(x) \boldsymbol{\Psi}^{\text {out }}=\left(\boldsymbol{U}(x) \boldsymbol{\Psi}_{1}\right) \stackrel{\text { out }}{\times} \ldots \stackrel{\text { out }}{\times}\left(\boldsymbol{U}(x) \boldsymbol{\Psi}_{n}\right) \\
& \boldsymbol{\varepsilon}_{p}\left(\rho_{n}, \ldots \rho_{1}\right) \cdot \boldsymbol{\Psi}^{\text {out }}=\boldsymbol{\Psi}_{p^{-1}(1)} \stackrel{\text { out }}{\times} \ldots \stackrel{\text { out }}{\times} \boldsymbol{\Psi}_{p^{-1}(n)} .
\end{aligned}
$$

But at this point of our analysis we have to take into account that $\boldsymbol{\Psi}^{\text {out }}$ might not only depend on the single particle states $\boldsymbol{\Psi}_{1}, \ldots \boldsymbol{\Psi}_{n}$ but also on the choice of the cones $\mathscr{S}_{1}, \ldots \mathscr{S}_{n}$. This apparent possibility is ruled out after the calculation of scalar products.

Let $\rho_{1}, \ldots \rho_{n} \in \Delta_{\mathscr{S}_{0}}$ and $\hat{\rho}_{1}, \ldots \hat{\rho}_{n} \in \Delta_{\mathscr{S}_{0}}$ be two sets of irreducible single particle representations such that either $\hat{\rho}_{i}=\rho_{i}, i=1, \ldots n$ or $\hat{\rho}_{j}$ is inequivalent to $\rho_{j}$ for some $j$. Then, as in relation (8.2), we can construct single particle creation operators $C_{1}(t e), \ldots C_{n}(t e)$ and $\hat{C}_{1}(t e), \ldots \hat{C}_{n}(t e)$ which are essentially localized in cones $\mathscr{S}_{1}+t\left(e+s_{1}\right), \ldots \mathscr{S}_{n}+t\left(e+s_{n}\right)$ and $\hat{\mathscr{S}}_{1}+t\left(e+\hat{s}_{1}\right), \ldots \hat{\mathscr{S}}_{n}+t\left(e+\hat{s}_{n}\right)$, respectively. According to the previous lemma there exist the limits

$$
\begin{aligned}
& \boldsymbol{\Psi}^{\text {out }}=s-\lim _{t \rightarrow \infty} \boldsymbol{C}_{1}(t e) \ldots \boldsymbol{C}_{n}(t e) \boldsymbol{\Omega}, \\
& \hat{\boldsymbol{\Psi}}^{\text {out }}=s-\lim _{t \rightarrow \infty} \hat{\boldsymbol{C}}_{1}(t e) \ldots \hat{\boldsymbol{C}}_{n}(t e) \boldsymbol{\Omega},
\end{aligned}
$$

provided the cones $\mathscr{S}_{1}, \ldots \mathscr{S}_{n}$ (respectively $\hat{\mathscr{S}}_{1}, \ldots \hat{\mathscr{S}}_{n}$ ) are spacelike separated.

Now if $T$ is any intertwiner from $\hat{\rho}_{n} \ldots \hat{\rho}_{1}$ to $\rho_{n} \ldots \rho_{1}$, then the scalar product $\left(\boldsymbol{\Psi}^{\text {out }} \boldsymbol{T} \hat{\boldsymbol{\Psi}}^{\text {out }}\right)$ can be expressed in terms of scalar products of the single particle states $\boldsymbol{\Psi}_{i}=\boldsymbol{C}_{i}(t e) \boldsymbol{\Omega}$ and $\hat{\boldsymbol{\Psi}}_{i}=\hat{\boldsymbol{C}}_{i}(t e) \boldsymbol{\Omega}$. To verify this let us first consider a situation where the cones $\mathscr{S}_{1}, \ldots \mathscr{S}_{n}$ and $\hat{\mathscr{S}}_{1}, \ldots \hat{\mathscr{S}}_{n}$ are arranged in a special way.

Lemma 8.2. Let $\hat{\mathscr{S}}_{i} \subset \mathscr{S}_{j}^{\prime}$ for $i \neq j$ and $i, j=1, \ldots n$. Then

$$
\left(\boldsymbol{\Psi}^{\text {out }}, \boldsymbol{T} \hat{\boldsymbol{\Psi}}^{\text {out }}\right)=\omega_{0}{ }^{\circ} \phi_{\rho_{1}}^{\mathscr{S}_{a}} \ldots \phi_{\rho_{n}}^{\mathscr{S}_{a}}(T) \cdot \prod_{i=1}^{n}\left(\boldsymbol{\Psi}_{i}, \hat{\boldsymbol{\Psi}}_{i}\right)
$$

if $\rho_{i}=\hat{\rho}_{i}$ for $i=1, \ldots$. If $\rho_{j} \nsucc \hat{\rho}_{j}$ for some $j$ the expression vanishes.

Proof. Applying the rules for computations with generalized fields it is easy to verify that

$$
\begin{aligned}
& \left(\boldsymbol{C}_{1}(t e) \ldots \boldsymbol{C}_{n}(t e) \boldsymbol{\Omega}, \boldsymbol{T} \cdot \hat{\boldsymbol{C}}_{1}(t e) \ldots \hat{\boldsymbol{C}}_{n}(t e) \boldsymbol{\Omega}\right) \\
& \quad=\left(\boldsymbol{\Omega}, \boldsymbol{T}^{\prime} \cdot \boldsymbol{C}_{n}(t e)^{\dagger} \ldots \boldsymbol{C}_{1}(t e)^{\dagger} \hat{\boldsymbol{C}}_{1}(t e) \ldots \hat{\boldsymbol{C}}_{n}(t e) \boldsymbol{\Omega}\right)
\end{aligned}
$$


where $T^{\prime}$ is some intertwiner from $\hat{\rho}_{n} \ldots \hat{\rho}_{1} \bar{\rho}_{1} \ldots \bar{\rho}_{n}$ to $l$. Because of the localization properties of the single particle creation operators at asymptotic times, one can rearrange the order of factors in the above expression, such that operators with asymptotically overlapping localization cones are placed next to each other. The result is, up to terms which vanish as $t \rightarrow \infty$,

$$
\left(\boldsymbol{\Omega}, \boldsymbol{T}^{\prime \prime} \cdot \boldsymbol{C}_{n}(t e)^{\dagger} \hat{\boldsymbol{C}}_{n}(t e) \ldots \boldsymbol{C}_{1}(t e)^{\dagger} \hat{\boldsymbol{C}}_{1}(t e) \boldsymbol{\Omega}\right),
$$

where $T^{\prime \prime}$ is an intertwiner from $\hat{\rho}_{1} \bar{\rho}_{1} \ldots \hat{\rho}_{n} \bar{\rho}_{n}$ to $l$. It is crucial now that the spacelike distance between the (essential) localization regions of the operator $\boldsymbol{C}_{1}(t e)^{\dagger} \hat{\boldsymbol{C}}_{1}(t e)$ and the remaining operators $\boldsymbol{C}_{i}(t e)^{\dagger} \hat{\boldsymbol{C}}_{i}(t e), i=2, \ldots n$ increases linearly with $t$. So taking into account that sp $\mathscr{U}$ has a lower mass-gap on $\mathscr{H}_{\hat{\rho}_{1} \bar{\rho}_{1}}$ and that the norms of the operators $C(t e)$ are bounded by a multiple of $|t|^{3 / 2}$ one can apply the clustertheorem as in [DHR II, Lemma 7.2] and insert to the left of $\boldsymbol{C}_{1}(t e)^{\dagger} \hat{\boldsymbol{C}}_{1}(t e)$ the projection $\boldsymbol{E}_{0}$ onto the translation invariant subspace of $\mathscr{H}_{\hat{\rho}_{1} \bar{\rho}_{1}}$. Neglecting terms which tend to zero as $t \rightarrow \infty$, this gives

$$
\left(\boldsymbol{\Omega}, \boldsymbol{T}^{\prime \prime} \boldsymbol{C}_{n}(t e)^{\dagger} \hat{\boldsymbol{C}}_{n}(t e) \ldots \boldsymbol{C}_{2}(t e)^{\dagger} \hat{\boldsymbol{C}}_{2}(t e) \boldsymbol{E}_{0} \boldsymbol{C}_{1}(t e)^{\dagger} \hat{\boldsymbol{C}}_{1}(t e) \boldsymbol{\Omega}\right) .
$$

Since $E_{0}=0$ if $\rho_{1}$ and $\hat{\rho}_{1}$ are inequivalent, this proves one half of the statement. If $\rho_{1}=\hat{\rho}_{1}$, then $E_{0}$ projects onto the ray of $\left\{\rho_{1} \bar{\rho}_{1}, R_{1} \Omega\right\}$, where $R_{1}$ is an isometric intertwiner from $l$ to $\rho_{1} \bar{\rho}_{1}$. So as in [DHR II] one finds after a straight forward calculation that the above expression is identical to

$$
\left(\boldsymbol{\Omega}, \boldsymbol{T}^{\prime \prime \prime} C_{n}(t e)^{\dagger} \hat{\boldsymbol{C}}_{n}(t e) \ldots \boldsymbol{C}_{2}(t e)^{\dagger} \hat{\boldsymbol{C}}_{2}(t e) \boldsymbol{\Omega}\right)\left(\boldsymbol{C}_{1}(t e) \boldsymbol{\Omega}, \hat{\boldsymbol{C}}_{1}(t e) \boldsymbol{\Omega}\right),
$$

where $T^{\prime \prime \prime}$ is an intertwiner from $\hat{\rho}_{2} \bar{\rho}_{2} \ldots \hat{\rho}_{n} \bar{\rho}_{n}$ to $l$. Repeating this procedure and bearing in mind that the vectors $\boldsymbol{C}(t e) \boldsymbol{\Omega}$ are $t$-independent single particle states one obtains in the limit $t \rightarrow \infty$

$$
\left(\boldsymbol{\Psi}^{\text {out }}, \boldsymbol{T} \hat{\boldsymbol{\Psi}}^{\text {out }}\right)=\varphi(T) \prod_{i=1}^{n}\left(\boldsymbol{\Psi}_{i}, \hat{\boldsymbol{\Psi}}_{i}\right)
$$

where $\varphi(T)$ is a number (an intertwiner from $l$ to $l$ ). The specific form of $\varphi(T)$ can be computed as in [DHR II]. We leave the details to the reader and remark that the result is obvious if all single particle representations are of pure Bose-or Fermitype, since $T$ is then a multiple of the identity. QED.

Now let $\mathscr{H}_{\text {p }}^{(1)}(\mathscr{S}, e)$ be the closed linear span of single particle states $\boldsymbol{C}(t e) \boldsymbol{\Omega}$, where in the defining relation $(8.2)$ for $C(t e)$ the testfunction $f$ varies within $\mathscr{L}_{\rho}(\mathscr{S}, e)$ and $\boldsymbol{B}=\{\rho, B\}$ within $\mathfrak{F}(\mathscr{S})$. Using the fact that $\mathfrak{F}(\mathscr{S}) \boldsymbol{\Omega}$ is dense in the fibers of $\mathscr{H}$ it is obvious that $\mathscr{H}_{\rho}^{(1)}(\mathscr{S}, e)$ is the subspace of single particle states $\Psi \in \mathscr{H}_{\rho}^{(1)}$ whose energy-momentum support is restricted by the condition

$$
\{p-(p e) e: p \in \operatorname{supp} \Psi\} \subset \mathscr{S}-a,
$$

$a$ being the apex of $\mathscr{S}$. So $\mathscr{H}_{\rho}^{(1)}(\mathscr{S}, e)$ describes particles whose spatial momenta $(p-(p e) e)$ are contained in $(\mathscr{S}-a) \cap e^{\perp}$, where $e^{\perp}$ denotes the spacelike plane orthogonal to $e$. Hence if $\mathscr{S}^{(k)}, k=1, \ldots m$ is any set of cones with common apex $a$ and if

$$
e^{\perp} \cap \bigcup_{k=1}^{m}\left(\mathscr{S}^{(k)}-a\right) \supset e^{\perp} \cap(\mathscr{S}-a)
$$


we get

$$
\sum_{k=1}^{m} \mathscr{H}_{\rho}^{(1)}\left(\mathscr{S}^{(k)}, e\right) \supset \mathscr{H}_{\rho}^{(1)}(\mathscr{S}, e)
$$

which means that in the construction of single particle states we can pass to smaller cones $\mathscr{S}$ without problems.

Coming back to the problem of calculating scalar products we are now almost finished: if $\mathscr{H}_{\rho_{n} \ldots \rho_{1}}^{\text {out }}\left(\mathscr{S}_{1}, \ldots \mathscr{S}_{n} ; e\right)$ denotes the closed linear span of vectors constructed in Lemma 8.1 we see from Lemma 8.2 that this space is isomorphic to the tensor product $\mathscr{H}_{\rho_{1}}^{(1)}\left(\mathscr{S}_{1}, e\right) \otimes \ldots \otimes \mathscr{H}_{\rho_{n}}^{(1)}\left(\mathscr{S}_{n}, e\right)$, the isomorphism being given by

$$
\boldsymbol{\Psi}_{1} \otimes \ldots \otimes \boldsymbol{\Psi}_{n} \rightarrow \boldsymbol{\Psi}_{1} \stackrel{\text { out }}{\times} \stackrel{\text { out }}{\times} \boldsymbol{\Psi}_{n} .
$$

Hence if $\mathscr{S}_{1}^{(1)}, \ldots \mathscr{S}_{1}^{(m)}$ is any set of spacelike cones satisfying relation (8.12) with $\mathscr{S}$ replaced by $\mathscr{S}_{1}$, and if each $\mathscr{S}_{1}^{(k)}$ is spacelike separated from $\mathscr{S}_{2}, \ldots \mathscr{S}_{n}$, then

$$
\sum_{k=1}^{m} \mathscr{H}_{\rho_{n} \ldots \rho_{1}}^{\text {out }}\left(\mathscr{S}_{1}^{(k)}, \mathscr{S}_{2}, \ldots \mathscr{S}_{n} ; e\right) \rightrightarrows \mathscr{H}_{\rho_{n} \ldots \rho_{1}}^{\text {out }}\left(\mathscr{S}_{1}, \ldots \mathscr{S}_{n} ; e\right)
$$

We now have the tools to compute the scalar products for arbitrary vectors which are constructed according to Lemma 8.1.

Theorem 8.3. Let $\quad \boldsymbol{\Psi}^{\text {out }}=\boldsymbol{\Psi}_{1} \stackrel{\text { out }}{\times} \ldots \stackrel{\text { out }}{\times} \boldsymbol{\Psi}_{n} \in \mathscr{H}_{\rho_{n} \ldots \rho_{1}}^{\text {out }}\left(\mathscr{S}_{1}, \ldots \mathscr{S}_{n} ; e\right)$ and $\hat{\boldsymbol{\Psi}}^{\text {out }}=$ $\hat{\boldsymbol{\Psi}}_{1}{ }^{\text {out }} \ldots \stackrel{\text { out }}{\times} \hat{\boldsymbol{\Psi}}_{n} \in \mathscr{H}_{\rho_{n} \ldots \rho_{1}}^{\text {out }}\left(\hat{\mathscr{S}}_{1}, \ldots \hat{\mathscr{S}}_{n} ; e\right)$ where the representations $\rho_{i}, \rho_{j} i, j=1, \ldots n$ are either equal or inequivalent. Then for any $T \in \rho_{n} \cdots \rho_{1}(\mathfrak{Q})^{\prime}$

$$
\left(\boldsymbol{\Psi}^{\text {out }}, \boldsymbol{T} \hat{\boldsymbol{\Psi}}^{\text {out }}\right)=\sum \omega_{0}{ }^{\circ} \phi_{\rho_{1}}^{\mathscr{S}_{a}} \ldots \phi_{\rho_{n}}^{\mathscr{S}_{a}}\left(T \varepsilon_{p}\left(\rho_{n}, \ldots \rho_{1}\right)\right) \prod_{i=1}^{n}\left(\boldsymbol{\Psi}_{i}, \hat{\boldsymbol{\Psi}}_{p(i)}\right),
$$

where the sum extends over all permutations $p \in P^{(n)}$ for which $\rho_{i}=\rho_{p(i)}, i=1, \ldots n$. Remark. $T$ is left arbitrary, because this result can then be used to calculate scalar products in any subrepresentation of $\rho_{n} \cdots \rho_{1}$.

Proof. Using relation (8.15) we can decompose $\boldsymbol{\Psi}^{\text {out }}$ and $\hat{\boldsymbol{\Psi}}^{\text {out }}$ into $\boldsymbol{\Psi}^{\text {out }}=\sum_{k=1}^{m} \boldsymbol{\Psi}_{k}^{\text {out }}$ and $\hat{\boldsymbol{\Psi}}^{\text {out }}=\sum_{k^{\prime}=1}^{m^{\prime}} \hat{\boldsymbol{\Psi}}_{k^{\prime}}^{\text {out }}$, where

$$
\boldsymbol{\Psi}_{k}^{\text {out }}=\boldsymbol{\Psi}_{1}^{(k)} \times \ldots \times \boldsymbol{\Psi}_{n}^{\text {out }} \in \mathscr{H}_{\rho_{n} \ldots \rho_{1}}^{\text {out }}\left(\mathscr{S}_{1}^{(k)}, \ldots \mathscr{S}_{n}^{(k)} ; e\right)
$$

and

$$
\hat{\boldsymbol{\Psi}}_{k^{\prime}}^{\text {out }}=\hat{\boldsymbol{\Psi}}_{1}^{\left(k^{\prime}\right)} \times \ldots \stackrel{\text { out }}{\text { out }} \hat{\boldsymbol{\Psi}}_{n}^{\left(k^{\prime}\right)} \in \mathscr{H}_{\rho_{n} \ldots \rho_{1}}^{\text {out }}\left(\hat{\mathscr{S}}_{1}^{\left(k^{\prime}\right)}, \ldots \hat{\mathscr{S}}_{n}^{\left(k^{\prime}\right)}, e\right) .
$$

The point is now, that for all $k$ and $k^{\prime}$ the cones $\mathscr{S}_{1}^{(k)}, \ldots \mathscr{S}_{n}^{(k)}$ and $\hat{\mathscr{S}}_{1}^{\left(k^{\prime}\right)}, \ldots \hat{\mathscr{S}}_{n}^{\left(k^{\prime}\right)}$ can be made so small such that they can be ordered into $n$ pairs, which are mutually spacelike separated. So for each $k, k^{\prime}$ there exists a permutation $p \in P^{(n)}$ for which

$$
\hat{\mathscr{S}}_{p(i)}^{\left(k^{\prime}\right)} \cup \mathscr{S}_{i}^{(k)} \subset\left(\hat{\mathscr{P}}_{p(j)}^{\left(k^{\prime}\right)} \cup \mathscr{S}_{j}^{(k)}\right)^{\prime}, i \neq j \quad i, j=1, \ldots n .
$$


But according to relation (8.9) we have

$$
\hat{\boldsymbol{\Psi}}_{k^{\prime}}^{\text {out }}=\varepsilon_{p}\left(\rho_{n}, \ldots \rho_{1}\right) \cdot\left(\hat{\boldsymbol{\Psi}}_{p(1)}^{\left(k^{\prime}\right)} \stackrel{\text { out }}{\times} \ldots \times \hat{\boldsymbol{\Psi}}_{p(n)}^{\left(k^{\prime}\right)}\right),
$$

so we are in a position to apply Lemma 8.2. The result is

$$
\left(\boldsymbol{\Psi}_{k}^{\text {out }}, \boldsymbol{T} \hat{\boldsymbol{\Psi}}_{k^{\prime}}^{\text {out }}\right)=\omega_{0}{ }^{\circ} \phi_{\rho_{1}}^{\mathscr{S}_{a}} \ldots \phi_{\rho_{n}}^{\mathscr{S}_{a}}\left(T \varepsilon_{p}\left(\rho_{n}, \ldots \rho_{1}\right)\right) \prod_{i=1}^{n}\left(\boldsymbol{\Psi}_{i}^{(k)}, \hat{\boldsymbol{\Psi}}_{p(i)}^{\left(k^{\prime}\right)}\right)
$$

if $\rho_{i}=\rho_{p(i)}$ for $i=1, \ldots n$; otherwise the expression vanishes. The statement follows then on summing over $k$ and $k^{\prime}$. QED.

Clearly, one can compute now also the scalar products for any linear combination of vectors which are constructed according to Lemma 8.1 (with arbitrary localization cones $\left.\mathscr{S}_{1}, \ldots \mathscr{S}_{n}\right)$. The closed linear span $\mathscr{H}_{\rho_{n} \ldots \rho_{1}}^{\text {out }}$ of all these vectors is the space of outgoing $n$-particle scattering states which are composed of single particle states from the representations $\rho_{1}, \ldots \rho_{n}$.

The only remaining question is whether our construction does depend on the choice of $e$ (of the Lorentz-system). That this is not the case can be seen as follows: the scattering states have been obtained as images of a linear mapping $\Omega^{\text {out }}(e)$ from $\mathscr{H}_{\rho_{1}}^{(1)} \otimes \ldots \otimes \mathscr{H}_{\rho_{n}}^{(1)}$ into $\mathscr{H}_{\rho_{n} \ldots \rho_{1}}^{\text {out }}$, which is bounded according to Theorem 8.3. Now let $e_{1}$ be another positive timelike direction and let $e(\xi)=c(\xi)\left((1-\xi) e+\xi e_{1}\right)$, $0 \leqq \xi \leqq 1$, be a path from $e$ to $e_{1}$, where $c(\xi)$ is a normalizing factor. Then we consider the space $\mathscr{D} \subset \mathscr{H}_{\rho_{1}}^{(1)} \otimes \ldots \otimes \mathscr{H}_{\rho_{n}}^{(1)}$ of vectors, whose wavefunctions $\psi_{n}\left(p_{1}, \ldots p_{n}\right)$ have compact support and vanish whenever the spatial parts $p-(p e(\xi)) e(\xi)$ of any two momenta $p_{i}, p_{j}$ become (almost) parallel for some $\xi$. Since the path $\xi \rightarrow e(\xi)$ lies on a 2 -dimensional plane it is obvious that $\mathscr{D}$ is dense in $\mathscr{H}_{\rho_{1}}^{(1)} \otimes \ldots \otimes \mathscr{H}_{\rho_{n}}^{(1)}$. On the other hand it follows from the second half of Lemma 8.1 that $\Omega^{\text {out }}(e(\xi))=\Omega^{\text {out }}(e)$ on $\mathscr{D}$, so taking into account that the mappings are bounded we find that $\Omega^{\text {out }}\left(e_{1}\right)=\Omega^{\text {out }}(e)$. This proves that our construction is independent of the Lorentz-system.

The space of incoming scattering states $\mathscr{H}_{\rho_{n} \ldots \rho_{1}}^{\text {in }}$ can be constructed in a similar way. But it is noteworthy that in the construction of creation operators $C(t e)$, which generate from $\boldsymbol{\Omega}$ single particle states in $\mathscr{H}_{\rho}^{(1)}(\mathscr{S}, e)$, one now has to use in the defining relation (8.2) operators $\boldsymbol{B}$ from $\mathfrak{F}(-\mathscr{S})$. So in contrast to local field theory there does not necessarily exist an interpolating field, which connects (the still existing) incoming and outgoing fields in the sense of the LSZ-asymptotic condition [28]. As a consequence, one cannot apply the reduction formulas, and the analytic structure of the $S$-matrix may differ from the familiar one.

Summing up, we have seen in the present section, that also for fields which are localized in cones a reasonable scattering theory exists. We emphasize, that the scattering states are uniquely characterized by the set of single particle states which they describe at asymptotic times. This means that the familiar interpretation of scattering experiments in terms of asymptotic observables does not require locality of interpolating fields. The same is true for the statistics of particles: a scattering state of particles belonging to a sector with pure Fermi-statistics, say, has a completely antisymmetric wave-function (relation 8.9), although the underlying fields might satisfy only the restricted spacelike commutation relations (5.9). So from this general point of view it seems worthwhile to devote further studies to these fields.

Another problem, which has not been discussed in our analysis but for which 
our results might be of some interest is the problem of asymptotic completeness. This problem consists actually of two parts: given a model, one has first to determine a (minimal) representation space for the observables containing all scattering states of the basic particles. In the second step one has then to verify that each vector in the representation space can be interpreted as a scattering state. The first part of this problem has been solved in this paper for massive theories with a conventional particle interpretation. There the set $(P)$ of particle representations describes all scattering states, and it is minimal in the sense that $(P)$ coincides (up to equivalence) with the set of representations which are induced by the scattering states. This result shows that a general solution of the difficult second part of the problem is impossible within the conventional framework of local field theory, if the existence of particles which require the introduction of string-like fields cannot be excluded.

\section{Conclusions}

Starting from the principle of locality of observables we have derived most of the qualitative features of massive particles which are observed in physics. Among the empirical facts not discussed is the relation between the spin and statistics of particles. Since we did not assume Lorentz-covariance, an answer to this problem lies outside the scope of this paper. But there might still be a multiplicity-statistics theorem in the present setting: in a sector with (para)-Fermi-statistics any particle multiplet should consist of an even number of states! In this context it is of interest, that in the decomposition

$$
\mathscr{H}^{(1)}=\int d^{3} p \mathscr{H}^{(1)}(\mathbf{p})
$$

of a single particle space with respect to the spatial momentum, (almost) all spaces $\mathscr{H}^{(1)}(\mathbf{p})$ have the same dimension, as a consequence of locality. So the notion of a particle multiplet does not require the presence of Lorentz-transformations. We are presently investigating these internal degrees of freedom of particles more closely.

For applications perhaps the most interesting result of our analysis is the insight that an explanation of the structural properties of particles does not require local charge carrying fields. Yet the localization properties of these fields are not completely arbitrary: as we have seen, it is always possible in a massive theory to find fields that are localized in a spacelike cone and connect the vacuum and the single particle states. Moreover, the location of this cone can be chosen at will.

The physical picture implied by this result is that a massive particle might be tied to an infinite string the direction of which is not visible. The presence of the string becomes manifest only in certain experiments such as the measurement of the total "electric" flux through the surface of a sufficiently large sphere surrounding the particle, or in the analytic structure of the $S$-matrix. As was discussed in the Introduction, this picture is only consistent if the vacuum state undergoes large loop-like fluctuations and if on the other hand large string-like fluctuations are suppressed.

In order to illustrate this heuristic picture we present a kinematic model which 
exhibits the described behaviour. Let $\mathbf{A}(\mathbf{x})$ be a vector-valued field on $\mathbb{R}^{3}$ which may be interpreted as a magnetic vector potential and let $\mathbf{E}(\mathbf{x})$ be the canonically conjugate electric field. We assume canonical commutation relations in the integrated and exponentiated Weyl-form

$$
e^{i \mathbf{E}(\mathbf{f})} e^{i \mathbf{A}(\mathbf{g})}=e^{i(\mathbf{f}, \mathbf{g})} e^{i \mathbf{A}(\mathbf{g})} e^{i \mathbf{E}(\mathbf{f})},
$$

where $\mathbf{E}(\mathbf{f}), \mathbf{A}(\mathbf{g})$ are the fields smoothed out with testfunctions $\mathbf{f}, \mathbf{g}$ with compact support, and

$$
(\mathbf{f}, \mathbf{g})=\int d^{3} x \mathbf{f}(\mathbf{x}) \cdot \mathbf{g}(\mathbf{x}) .
$$

The algebra $\mathfrak{H}$ generated by the unitary operators $e^{i \mathbf{E}(\mathbf{f})} e^{i \mathbf{A}(\mathbf{g})}$ has a natural local structure which is induced by the local structure in the testfunction space: if $\mathscr{R} \subset \mathbb{R}^{3}$ we define $\mathfrak{U}(\mathscr{R})$ as the algebra which is generated by the above unitaries with supp f, supp $\mathbf{g} \subset \mathscr{R}$. Similarly we can define translations $\alpha_{\mathbf{x}}$ on $\mathfrak{A}$, setting

$$
\alpha_{\mathbf{x}}\left(e^{i \mathbf{E}(\mathbf{f})} e^{i \mathbf{A}(\mathbf{g})}\right)=e^{i \mathbf{E}\left(\mathbf{f}_{\mathbf{x}}\right)} e^{i \mathbf{A}\left(\mathbf{g}_{\mathbf{x}}\right)},
$$

where $\mathbf{f}_{\mathbf{x}}, \mathbf{g}_{\mathbf{x}}$ are the translated testfunctions.

Now let us consider the state $\omega_{0}$ on $\mathfrak{H}$ which is determined by the conditions

$$
\omega_{0}\left(e^{i \mathbf{E}(\mathbf{f})} \cdot e^{i \mathbf{A}(\mathbf{g})}\right)=\left\{\begin{array}{l}
1 \text { if } \text { curl } \mathbf{f}=0, \operatorname{div} \mathbf{g}=0 \\
0 \text { otherwise. }
\end{array}\right.
$$

One can show that $\omega_{0}$ is a pure state which is invariant under translations, and we will take $\omega_{0}$ as the vacuum in our kinematic model. Next we introduce states $\omega_{\mathbf{h}}$ on $\mathfrak{A}$ by

$$
\omega_{\mathbf{h}}\left(e^{i \mathbf{E}(\mathbf{f})} \cdot e^{i \mathbf{A}(\mathbf{g})}\right)=e^{i(\mathbf{h}, \mathbf{f})} \cdot \omega_{0}\left(e^{i \mathbf{E}(\mathbf{f})} \cdot e^{i \mathbf{A}(\mathbf{g})}\right),
$$

where $\mathbf{h}$ is an infinitely differentiable vector-valued function such that div $\mathbf{h}$ has compact support. The following facts can then easily be established:

i) $\omega_{\mathbf{h}}$ and $\omega_{\mathbf{h}^{\prime}}$ induce equivalent representations $\pi_{\mathbf{h}}$ and $\pi_{\mathbf{h}^{\prime}}$ of $\mathfrak{Y}$ if and only if

$$
\int d^{3} x \operatorname{div} \mathbf{h}(\mathbf{x})=\int d^{3} x \operatorname{div} \mathbf{h}^{\prime}(\mathbf{x})
$$

Therefore, states $\omega_{\mathbf{h}}$ belonging to different sectors can be distinguished by their "electric" charge.

ii) For all admissible functions $\mathbf{h}$ and arbitrary spatial cones $\mathscr{S} \subset \mathbb{R}^{3}$ one has

$$
\pi_{\mathbf{h}}\left\lceil\mathfrak{U}^{c}(\mathscr{S}) \simeq \pi_{0} \uparrow \mathfrak{U}^{c}(\mathscr{S}) .\right.
$$

If in particular $\mathscr{S} \supset \operatorname{supp}(\operatorname{div} \mathbf{h})$ then

$$
\omega_{\mathbf{h}}(A)=\omega_{0}(A) \text { for } A \in \mathfrak{U}^{c}(\mathscr{S})
$$

Hence the direction of the electric flux lines cannot be observed.

iii) If $\int d^{3} x \operatorname{div} \mathbf{h}(\mathbf{x}) \neq 0$, then the representations $\pi_{\mathbf{h}}$ and $\pi_{0}$ restricted to the algebra of the complement $\mathcal{O}^{c}$ of any bounded region $\mathcal{O} \subset \mathbb{R}^{3}$ are disjoint,

$$
\pi_{\mathbf{h}}\left\lceil\mathfrak{U}\left(\mathcal{O}^{c}\right) \mid \pi_{0}\left\lceil\mathfrak{U}\left(\mathcal{O}^{c}\right)\right.\right.
$$

This is a consequence of Gauss' law and expresses the fact that an electric charge cannot be localized in a bounded region. 
Thus this primitive model displays the desired features. The enhancement of loops and suppression of strings is very drastic in this example and there exist refinements with a somewhat more realistic behaviour. We do not expect, however, that such a situation is dynamically possible in theories with a continuous Abelian gauge group, even in the presence of massless particles [29].

A more realistic model could be the $\mathbb{Z}_{2}$-lattice gauge theory coupled to a $\mathbb{Z}_{2}$ valued Higgs field. The pure gauge theory is self-dual in four dimensions [30], so the weak coupling region is accessible from the strong coupling region by the duality transformation. In the pure gauge theory with external charges stringlike localized sectors seem to exist in the weak coupling region, and it is anteresting question whether this situation remains unchanged if one adds weakly coupled Higgs fields.

Even more interesting is the question of whether stringlike localizable particles exist in non-Abelian gauge theories, such as quantum chromodynamics. There are heuristic arguments that quarks, if they exist as isolated particles, cannot be localizable in finitely extended regions. Recently West [31] showed under the assumption that dispersion relations can be applied as in local field theory that quarks are confined in quantum chromodynamics. But in view of the present results one should take the possibility of string-like localizable quarks more seriously into account in a thorough investigation of the confinement problem.

To get a convenient description of theories with such particles one could try to develop a Wightman-type formalism for fields living on strings. At present not much is known about the properties of such theories, and one can only speculate that they might exhibit new and interesting features. For example, the no-go theorems ruling out interacting theories without particle production in four dimensions [32] use locality of the interpolating fields as an essential ingredient. So it seems to be worthwhile to reinvestigate the possibilities of theories without particle production, with factorizing S-matrices, with an infinite number of conservation laws or even completely integrable theories under this new aspect. In this context it is encouraging that string-like fields in three dimensions (which have not been discussed in this paper) exhibit similar topological properties as the soliton fields in two dimensions.

Speculating even further, there may exist models where the fundamental particles (the quarks) are string-like localizable and cannot be produced in collisions, whereas their finitely localizable bound states can be freely produced and annihilated. For the quarks there would then be no experimental sources, and in this sense they would appear to be confined. But nevertheless, such quarks could be present in the physical world and be observed! This picture does not even seem to be in obvious conflict with the qualitative features of present-day physics [33].

\section{Appendix}

We give here the proof of Theorem 3.1. In order to display how the dimension of space enters into the construction we work in arbitrary $(s \geqq 1)$ spatial dimensions. 
1. Given $\tilde{p}, \tilde{p}$ one chooses points $k_{0}, k_{1}, \ldots k_{s} \in \mathbb{R}^{s+1}$ for which the following conditions hold:

$$
\begin{aligned}
& \tilde{p_{1}}+\sum_{i=0}^{j} k_{i} \in H_{m}=\left\{p: p^{2}=m^{2}, p_{0}>0\right\}, \quad j=0,1, \ldots s-1 \\
& \tilde{p_{1}}+\sum_{i=0}^{s} k_{i}=\tilde{p_{2}} \\
& \tilde{p_{1}}+k_{j} \in\left\{p: p^{2}<0\right\}, \quad j=1, \ldots s .
\end{aligned}
$$

(That such a set of points exists can easily be seen if one goes to the centre of mass system of $p_{1}^{\sim}$ and $\tilde{p_{2}}$.). Since the hyperboloid $H_{m} \subset \mathrm{sp} \mathscr{U}$ is isolated from the rest of sp $\mathscr{U}$ and since the set $\left\{p: p^{2}<0\right\}$ is open and $\left\{p: p^{2}<0\right\} \cap \operatorname{sp} \mathscr{U}=\emptyset$ there exist also neighbourhoods $\Gamma_{i}$ of $k_{i}, i=0,1 \ldots s$ and a neighbourhood $\Delta^{\sim}$ of $p_{1}^{\sim}$ such that

$$
\begin{aligned}
& \Delta^{\sim} \subset \Delta_{1},\left(\Delta^{\sim}+\sum_{i=0}^{s} \Gamma_{i}\right) \subset \Delta_{2}, \\
& \left(\Delta^{\sim}+\sum_{i=0}^{j} \Gamma_{i}\right) \cap \operatorname{sp} \mathscr{U} \subset H_{m} ; j=0,1, \ldots s, \\
& \left(\Delta^{\sim}+\Gamma_{j}\right) \cap \operatorname{sp} \mathscr{U}=\emptyset, \quad j=1, \ldots s .
\end{aligned}
$$

For the next step in the argument it is convenient to adjust also the sizes of $\Delta^{\sim}$ and $\Gamma_{i}$ according to

$$
\Delta^{\sim}+\sum_{i=0}^{j} \Gamma_{i} \subset \operatorname{sp} \mathscr{U}-\Gamma_{j+1}, \quad j=0,1, \ldots s-1 .
$$

2. Then one chooses almost local operators $B_{i}$ with momentum transfer $\Gamma_{i}$, $i=0,1, \ldots$ s such that

$$
B_{s} B_{s-1} \ldots B_{0} E\left(\Delta^{\sim}\right) \neq 0 \text {. }
$$

This is possible because if $B_{j+1} B_{j} \ldots B_{0} E\left(\Delta^{\sim}\right)=0$ for all almost local operators $B_{j+1}$ with momentum transfer $\Gamma_{j+1}$, then also $E\left(\operatorname{sp} \mathscr{U}-\Gamma_{j+1}\right) B_{j} \ldots B_{0} E\left(\Delta^{\sim}\right)=0$ (cf. the proof of Proposition 2.2). Taking into account that the regions $\Gamma_{i}$ have been adjusted such that the range of the operator $B_{j} \ldots B_{0} E\left(\Delta^{\sim}\right)$ is contained in $E\left(\right.$ sp $\left.\mathscr{U}-\Gamma_{j+1}\right) \mathscr{H}$, one concludes that $B_{j} \ldots B_{0} E\left(\Delta^{\sim}\right)=\emptyset$, and repeating the argument one is led to a contradiction.

3. Next one considers the operator-valued function

$$
\mathbf{x}_{1}, \ldots \mathbf{x}_{s} \rightarrow B_{s}\left(\mathbf{x}_{s}\right) \ldots B_{1}\left(\mathbf{x}_{1}\right) B_{0} \cdot E\left(\Delta^{\sim}\right) .
$$

Since the operators $B_{i}$ have bounded energy-momentum transfer, all functions $x \rightarrow B_{i}(x)$ and therefore also the above function are continuous in the uniform topology on $\mathscr{B}(\mathscr{H})$. Moreover, since $\left(\Delta^{\sim}+\Gamma_{i}\right) \cap \operatorname{sp} \mathscr{U}=0$ for $i=1, \ldots s$ one has $B_{i} E\left(\Delta^{\sim}\right)=0$, hence

$$
\begin{aligned}
& B_{s}\left(\mathbf{x}_{s}\right) \ldots B_{1}\left(\mathbf{x}_{1}\right) B_{0} E\left(\Delta^{\sim}\right) \\
& \quad=\left[B_{s}\left(\mathbf{x}_{s}\right),\left[\ldots,\left[B_{1}\left(\mathbf{x}_{1}\right), B_{0}\right] \ldots\right]\right] E\left(\Delta^{\sim}\right) .
\end{aligned}
$$


Bearing in mind that the operators $B_{j}, j=0,1 \ldots s$ are almost local, one obtains the bound

$$
\left\|\left[B_{s}\left(\mathbf{x}_{s}\right),\left[\ldots,\left[B_{1}\left(\mathbf{x}_{1}\right), B_{0}\right] \ldots\right]\right]\right\| \leqq h\left(\sum_{i=1}^{s}\left|\mathbf{x}_{i}\right|\right)
$$

and consequently

$$
\left\|B_{s}\left(\mathbf{x}_{s}\right) \ldots B_{1}\left(\mathbf{x}_{1}\right) B_{0} E\left(\Delta^{\sim}\right)\right\| \leqq h\left(\sum_{i=1}^{s}\left|\mathbf{x}_{i}\right|\right)
$$

where $h$ is rapidly decreasing. So the Fourier transform

$$
\mathbf{k}_{1}, \ldots \mathbf{k}_{s} \rightarrow \int \prod_{j=1}^{s} d^{s} x_{j} e^{-i\left(\mathbf{k}_{j} \mathbf{x}_{j}\right)} B_{s}\left(\mathbf{x}_{s}\right) \ldots B_{1}\left(\mathbf{x}_{1}\right) B_{0} E\left(\Delta^{\sim}\right)
$$

is a uniformly continuous function in $\mathscr{B}(\mathscr{H})$, which is not identical to 0 since $B_{s} \ldots B_{1} B_{0} E\left(\Delta^{\sim}\right) \neq 0$. In particular, there exist $s$ linearly independent vectors $\mathbf{q}_{1}, \ldots \mathbf{q}_{s}$ for which this function does not vanish, and these vectors are kept fixed in the following. Then one defines the operator

$$
B^{a}=\int \prod_{j=1}^{s} d^{s} x_{j} e^{-i\left(\mathbf{q}_{j} \mathbf{x}_{j}\right)}\left[B_{s}\left(\mathbf{x}_{s}\right),\left[\ldots,\left[B_{1}\left(\mathbf{x}_{1}\right), B_{0}\right] \ldots\right]\right],
$$

which is again almost local and satisfies $B^{a} E\left(\Delta^{\sim}\right) \neq 0$ according to the above remarks. $B^{a}$ is essentially the operator $B$ of the theorem, which will be shown in the remainder of this proof.

4. If $g_{1}, \ldots g_{s}$ are smooth functions on $\mathbb{R}^{s}$ then the norm of the operator-valued function

$$
\mathbf{x}_{1}, \ldots \mathbf{x}_{s} \rightarrow B_{s}\left(\mathbf{x}_{s}\right) g_{s}(\mathbf{P}) B_{s-1}\left(\mathbf{x}_{s-1}\right) \ldots B_{1}\left(\mathbf{x}_{1}\right) g_{1}(\mathbf{P}) B_{0} E\left(\Delta^{\sim}\right)
$$

converges also rapidly to 0 if $\sum_{i=1}^{s}\left|\mathbf{x}_{i}\right|$ tends to infinity. To verify this the following two remarks may suffice: first one can replace everywhere in the above expression the functions $g_{j}$ by testfunctions with compact support because of the restricted energy-momentum transfer of the operators $B_{j}$. And second the norm of

$$
\begin{aligned}
& \mathbf{x}_{1}, \ldots \mathbf{x}_{s}, \mathbf{y}_{1}, \ldots \mathbf{y}_{s} \rightarrow \\
& f_{s}\left(\mathbf{y}_{s}\right) \ldots f_{1}\left(\mathbf{y}_{1}\right) B_{s}\left(\mathbf{x}_{s}\right) U\left(\mathbf{y}_{s}\right) B_{s-1}\left(\mathbf{x}_{s-1}\right) \ldots B_{1}\left(\mathbf{x}_{1}\right) U\left(\mathbf{y}_{1}\right) B_{0} E\left(\Delta^{\sim}\right),
\end{aligned}
$$

where $f_{1}, \ldots f_{s}$ are arbitrary testfunctions, converges rapidly to zero if $\sum_{i=1}^{s}\left(\left|\mathbf{x}_{i}\right|+\left|\mathbf{y}_{i}\right|\right)$ tends to infinity, because of $B_{i}(\mathbf{x}) U(\mathbf{y})=U(\mathbf{y}) B_{i}(\mathbf{x}-\mathbf{y})$ and the bounds given in the previous step. Hence if one integrates this expression with respect to $\mathbf{y}_{1}, \ldots \mathbf{y}_{s}$ the statement follows. By the same argument and a change of variables one can also show that

$$
\begin{aligned}
& \int \prod_{j=1}^{s} d^{s} x_{j} e^{-i\left(\mathbf{q}_{j} \mathbf{x}_{j}\right)} B_{s}\left(\mathbf{x}_{s}\right) g_{s}(\mathbf{P}) B_{s-1}\left(\mathbf{x}_{s-1}\right) \ldots B_{1}\left(\mathbf{x}_{1}\right) g_{1}(\mathbf{P}) B_{0} E\left(\Delta^{\sim}\right) \\
& \quad=g_{s}\left(\mathbf{P}+\mathbf{q}_{s} \cdot 1\right) g_{s-1}\left(\mathbf{P}+\left(\mathbf{q}_{s}+\mathbf{q}_{s-1}\right) \cdot 1\right) \ldots g_{1}\left(\mathbf{P}+\sum_{i=1}^{s} \mathbf{q}_{i} \cdot 1\right) B^{a} E\left(\Delta^{\sim}\right),
\end{aligned}
$$


where $B^{a}$ is the operator defined in step 3 .

5. It is convenient to introduce the notation $\varepsilon(\mathbf{p})=\left(|\mathbf{p}|^{2}+m^{2}\right)^{1 / 2}$ and to distinguish the following functions of the momentum operator:

$$
V_{\mathbf{q}}(t)=\int e^{i t \varepsilon(\mathbf{p}+\mathbf{q})} d E(p)
$$

Clearly, $V_{0}(t)$ acts on $\mathscr{H}^{(1)}$ like the time-translations $U(t)$,

$$
U(t) \uparrow \mathscr{H}^{(1)}=V_{\mathbf{0}}(t) \uparrow \mathscr{H}^{(1)} \text {. }
$$

Taking into account that $E\left(\Delta^{\sim}+\sum_{i=0}^{j} \Gamma_{i}\right) \mathscr{H} \subset \mathscr{H}^{(1)}$ for $j=0,1 \ldots s$ as well as the result of step 4 one obtains

$$
\begin{aligned}
& \int \prod_{j=1}^{s} d^{s} x_{j} e^{-i\left(\mathbf{q}_{j}, \mathbf{x}_{j}\right)} B_{s}\left(x_{s}\right) \ldots B_{1}\left(x_{1}\right) B_{0} E\left(\Delta^{\sim}\right) \\
& \quad=V_{0}\left(t_{s}\right) V_{\mathbf{q}_{s}}\left(t_{s-1}-t_{s}\right) \ldots V \sum_{i=2}^{s} \mathbf{q}_{i}\left(t_{1}-t_{2}\right) V \sum_{i=1}^{s} \mathbf{q}_{i}\left(-t_{1}\right) B^{a} E\left(\Delta^{\sim}\right),
\end{aligned}
$$

where $x_{i}=\left(t_{i}, \mathbf{x}_{i}\right)$. Hence if one puts

$$
B_{f}^{a}=\int \prod_{j=1}^{s} d^{s+1} x_{j} e^{-i\left(\mathbf{q}_{j} \mathbf{x}_{j}\right)} f\left(t_{s}, \ldots t_{1}\right)\left[B_{s}\left(x_{s}\right),\left[\ldots,\left[B_{1}\left(x_{1}\right), B_{0}\right] \ldots\right]\right]
$$

for any testfunction $f$ on $\mathbb{R}^{s}$, one arrives at

$$
B_{f}^{a} \cdot E\left(\Delta^{\sim}\right)=\tilde{f}\left(Q_{s}, \ldots Q_{1}\right) B^{a} E\left(\Delta^{\sim}\right)
$$

Here $\tilde{f}$ is the Fourier transform of $f$ and

$$
Q_{i}=\varepsilon\left(\mathbf{P}+\sum_{j=i}^{s+1} \mathbf{q}_{j} \cdot 1\right)-\varepsilon\left(\mathbf{P}+\sum_{j=i+1}^{s+1} \mathbf{q}_{j} \cdot 1\right), \quad i=1, \ldots s
$$

with the convention $\mathbf{q}_{s+1}=0$. Since $f$ converges rapidly to 0 for large arguments $B_{f}^{a}$ is again an almost local operator.

6. In order to find out which functions of the momentum operator $\mathbf{P}$ can be obtained by varying $f$ in $\tilde{f}\left(Q_{s}, \ldots Q_{1}\right)$ one has to study the mapping $\sigma$ of $\mathbb{R}^{s}$ into $\mathbb{R}^{s}$ which is defined in terms of the coordinates $p_{i}$ of $\mathbf{p}$ by

$$
p_{i} \rightarrow \varepsilon\left(\mathbf{p}+\sum_{j=i}^{s+1} \mathbf{q}_{j}\right)-\varepsilon\left(\mathbf{p}+\sum_{j=i+1}^{s+1} \mathbf{q}_{j}\right), \quad i=1, \ldots s .
$$

Since the function $\mathbf{k} \rightarrow \varepsilon(\mathbf{k})$ is analytic in a complex neighbourhood of $\mathbb{R}^{s} \sigma$ is smooth. Moreover the determinant of the Jacobian of $\sigma$ is also analytic in a neighbourhood of $\mathbb{R}^{s}$, and its value at $\mathbf{p}=0$ can easily be seen to be different from 0 because the vectors $\mathbf{q}_{i}, i=1, \ldots s$ are linearly independent (step 3 ). So the Jacobian of $\sigma$ can only be singular on a closed set $\Delta_{0} \subset \mathbb{R}^{s}$ of Lebesgue measure 0 .

Now $E\left(\mathbb{R} \times \Delta_{0}\right)=0$ because the spectrum of $\mathbf{P}$ is absolutely continuous (cf. Proposition 2.2), hence in view of $B^{a} E\left(\Delta^{\sim}\right) \neq 0$ there exists a point $\mathbf{r}$ in the complement of $\boldsymbol{\Delta}_{0}$ such that $E(\mathbb{R} \times \boldsymbol{\Delta}) B^{a} E\left(\boldsymbol{\Delta}^{\sim}\right) \neq 0$ for each neighbourhood $\boldsymbol{\Delta}$ of $\mathbf{r}$. On the other hand $\sigma$ is regular at $\mathbf{r}$, and according to the inverse function theorem there exists a neighbourhood $\boldsymbol{\Delta}$ of $\mathbf{r}$ such that $\sigma$ is an invertible map of $\boldsymbol{\Delta}$ onto $\sigma(\boldsymbol{\Delta})$ with 
a smooth inverse $\sigma^{-1}$. So given any testfunction $\tilde{f}$ with support in $\Delta$ one can define a testfunction $\tilde{f}_{\sigma}$ with support in $\sigma(\Delta)$ by setting $\tilde{f}_{\sigma}=\tilde{f}^{\circ} \sigma^{-1}$ on $\sigma(\Delta)$ and $\tilde{f}_{\sigma}=0$ on the complement of $\sigma(\Delta)$. With this definition one gets

$$
B_{f_{\sigma}}^{a} E\left(\Delta^{\sim}\right)=\tilde{f}(\mathbf{P}) \cdot B^{a} E\left(\Delta^{\sim}\right)
$$

for the restricted class of testfunctions $\tilde{f}$, and since $E(\mathbb{R} \times \Delta) B^{a} E\left(\Delta^{\sim}\right) \neq 0$ there exists in particular a testfunction $\tilde{f}_{a}$ with support in $\boldsymbol{\Delta}$ such that $\tilde{f}_{a}(\mathbf{P}) \cdot B^{a} \cdot E\left(\Delta^{\sim}\right) \neq 0$.

7. In order to extend this result to arbitrary functions one proceeds as follows: given a smooth function $g$ on $\mathbb{R}^{s+1}$ one restricts it to the hyperboloid $H_{m}=$ $\left\{p: p_{0}=\left(\mathbf{p}^{2}+m^{2}\right)^{1 / 2}\right\}$ which is parameterized by the momentum $\mathbf{p}$; this gives a smooth function $\tilde{g}$ on $\mathbb{R}^{s}$. Then one multiplies $\tilde{g}$ with the function $\tilde{f_{a}}$ introduced in step 6; the product $\tilde{g} \tilde{f}_{a}$ is a testfunction with support in $\Delta$, so $\left(\tilde{g} \tilde{f}_{a}\right)_{\sigma}$ is defined. Finally, one takes the inverse Fourier transform $\mathscr{F}^{-1}$ of the latter function. The result is a testfunction $L(g)$ on $\mathbb{R}^{s}$, formally given by

$$
L(g)=\mathscr{F}^{-1} \circ\left(\tilde{g} \tilde{f}_{a}\right)_{\sigma}, \quad \tilde{g}=g \nmid H_{m} .
$$

From the previous calculations it follows then that

$$
B_{L(g)}^{a} E\left(\Delta^{\sim}\right)=\tilde{g}(\mathbf{P}) \tilde{f}_{a}(\mathbf{P}) B^{a} E\left(\Delta^{\sim}\right)=\tilde{g}(\mathbf{P}) B_{L(e)}^{a} E\left(\Delta^{\sim}\right),
$$

where $e$ is the constant function $e(p)=1$. Since the range of $B_{L(e)}^{a} E\left(\Delta^{\sim}\right)$ is contained in $\mathscr{H}^{(1)}$ one can replace in this equation $\tilde{g}(\mathbf{P})$ by $g(P)$, so

$$
B_{L(g)}^{a} E\left(\Delta^{\sim}\right)=g(P) B_{L(e)}^{a} E\left(\Delta^{\sim}\right) .
$$

Also

$$
B_{L(e)}^{a} E\left(\Delta^{\sim}\right)=f_{a}(\mathbf{P}) B^{a} E\left(\Delta^{\sim}\right) \neq 0,
$$

and therefore the family of almost local operators $B_{L(g)}^{a}$ satisfies the first two relations of the theorem.

8. The remaining relations can be fulfilled if one adjusts the region $\Delta^{\sim}$ and the momentum transfer of the operators $B_{L(g)}^{a}$ as follows: first one chooses some region $\Delta_{1}^{\tilde{1}}$, such that the closure of $\Delta_{1}^{\tilde{1}}$ is contained in $\Delta^{\sim}$ and $B_{L(e)}^{a} E\left(\Delta_{1}^{\tilde{1}}\right) \neq 0$. (This is possible because $B_{L(e)}^{a} E\left(\Delta_{1}^{\tau}\right) \neq 0$.) So the Fourier transform of $x \rightarrow B_{L(e)}^{a}(x) E\left(\Delta_{1}^{\tilde{r}}\right)$ is different from 0 and there exists a testfunction $f_{\Gamma}$ whose Fourier transform has support in a region $\Gamma$ such that $\tilde{\Delta_{1}}+\Gamma-\Gamma \subset \Delta^{\sim}$ and

$$
\int d^{s+1} x f_{\Gamma}(x) B_{L(e)}^{a}(x) E\left(\Delta_{1}^{\tilde{r}}\right) \neq 0 .
$$

Then one defines

$$
B_{g}=\int d^{s+1} x f_{\Gamma}(x) B_{L(g)}^{a}(x)
$$

and $B_{e}=B$. Clearly $B_{g}$ is an almost local operator for any smooth function $g$. Also, $B \cdot E\left(\Delta_{1}^{\tilde{1}}\right) \neq 0$ and

$$
\begin{aligned}
B_{g} \cdot E\left(\Delta^{\sim}\right) & =\int d^{s+1} x f_{\Gamma}(x) U(x) B_{L(g)}^{a} E\left(\Delta^{\sim}\right) U(x)^{-1} \\
& =g(P) \cdot B E\left(\Delta^{\sim}\right) .
\end{aligned}
$$

Since $\Delta_{1}^{\tilde{C}} \subset \Delta^{\tilde{4}}$, this relation holds a fortiori if one replaces $\Delta^{\sim}$ by $\Delta_{1}^{\tilde{1}}$. Furthermore, 
setting $\Delta_{2}^{\tilde{2}}=\Delta_{1}^{\tilde{1}}+\Gamma$, the range of $B_{g} \cdot E\left(\Delta_{1}\right)$ is contained in $E\left(\Delta_{2}^{\sim}\right) \mathscr{H}$, and taking into account that $\Delta_{2}^{\sim}-\Gamma=\Delta_{1}^{\sim}+\Gamma-\Gamma \subset \Delta^{\sim}$ one gets

$$
E\left(\Delta_{2}^{\sim}\right) B_{g}=E\left(\Delta_{2}^{\sim}\right) B_{g} E(\Delta)=E\left(\Delta_{2}^{\sim}\right) g(P) B,
$$

which is relation (iii) of the theorem. Finally, bearing in mind that in the process of constructing $B_{g}$ one has only restricted the momentum transfer of operators and the support of projections, it is also clear that $\tilde{\Delta_{1}} \subset \Delta_{1}$ and $\tilde{\Delta}_{2}^{\sim} \subset \Delta_{2}$. So the family of operators $B_{g}$ has all the desired properties, and this completes the proof.

From the above argument it follows also that $g \rightarrow B_{g}$ is a linear mapping which depends continuously (in the topology of $\mathscr{C}^{\infty}$ ) on $g$. Hence if $B^{\prime}$ is an arbitrary local or almost local operator in $\mathscr{B}(\mathscr{H})$ one can define

$$
\left(B^{\prime} \cdot B\right)_{\tilde{f}}=\int d^{s+1} x f(x) B^{\prime}(x) \cdot B_{e_{x}},
$$

where $f$ is any testfunction on $\mathbb{R}^{s+1}, B_{e_{x}}$ is the operator which is attached to the function $e_{x}(p)=\exp (i p x)$, and the integral is defined as a weak integral. It is easy to verify that $\left(B^{\prime} \cdot B\right)_{\tilde{f}}$ is an almost local operator and that

$$
\tilde{f}(P) \cdot B^{\prime} B E\left(\Delta_{1}^{\sim}\right)=\left(B^{\prime} B\right)_{\tilde{f}} E\left(\Delta_{1}^{\tilde{1}}\right) .
$$

Therefore one may relax in Theorem 3.1 the condition that the range of $B \cdot E\left(\Delta_{1}^{\sim}\right)$ is contained in $\mathscr{H}^{(1)}$. As a matter of fact, one can reach any spectral subspace $E(\Delta) \mathscr{H}$ by a suitable choice of $B$.

Acknowledgement. We dedicate this article to the memory of Jorge Andre Swieca, whose structural theorem on charges in Abelian gauge theories [1] stimulated these investigations. We also profited from valuable suggestions of Rudolf Haag, which ultimately led us to the proof of Theorem 3.1, and from useful discussions with Hans Jürgen Borchers, Sergio Doplicher, Gerhard Mack and John E. Roberts.

\section{References}

1. Swieca, J. A. : Charge screening and mass spectrum, Phys. Rev. D13, 312 (1976)

2. Streater, R. F., Wightman, A. S. : PCT, spin and statistics and all that, New York: Benjamin Inc 1964

3. Doplicher, S., Haag, R., Roberts, J. E. : Local observables and particle statistics I. Commun. Math. Phys. 23, 199 (1971)

4. Doplicher, S., Haag, R., Roberts, J. E. : Local observables and particle statistics II. Commun. Math. Phys. 35, 49 (1974)

5. Strocchi, F., Wightman, A. S. : Proof of the charge superselection rule in local relativistic quantum field theory, J. Math. Phys. 15, 2198 (1974)

6. Buchholz, D., Fredenhagen, K. : Charge screening and mass spectrum in Abelian gauge theories, Nucl. Phys. B154, 226 (1979)

7. Haag, R., Kastler, D. : An algebraic approach to field theory, J. Math. Phys. 5, 848 (1964)

8. Wilson, K. : Confinement of quarks, Phys. Rev. D10, 2445 (1974)

9. Sakai, S. : $C^{*}$-algebras and $W^{*}$-algebras, Berlin, Heidelberg, New-York: Springer 1971

10. Borchers, H. J. : On the vacuum state in quantum field theory, Commun. Math. Phys. 1, 57 (1965)

11. Fröhlich, J., Morcchio G., Strocchi, F. : Charged sectors and scattering states in Quantum Electrodynamics, Ann. Phys. 119, 241 (1979)

12. Buchholz, D. : (to be published)

13. Jost, R. : The general theory of quantized fields. Providence, Rhode Island: Am. Math. Soc., 1965 
14. Hepp, K., Jost, R. : Über die Matrixelemente des Translationsoperators, Helv. Phys. Acta 35, 34 (1962)

15. Borchers, H. J., Buchholz D. : (to be published)

16. Newton, T. D., Wigner, E. P. : Localized states for elementary systems, Rev. Mod. Phys. 21, 400 (1949)

17. Fredenhagen, K.: On the existence of antiparticles, Commun. Math. Phys. 79, 141 (1981)

18. Bisognano, J. J., Wichmann, E. : On the duality condition for a hermitean scalar field. J. Math. Phys. 16, 985-1007 (1975)

19. Borchers, H. J. : Energy and momentum as observables in quantum field theory, Commun. Math. Phys. 2, 49 (1966)

20. Vladimirov, V. S. : Methods of the theory of functions of many complex variables; Cambridge, Massachusetts and London: MIT Press 1966

21. Borchers, H. J.: On the converse of the Reeh-Schlieder theorem, Commun. Math. Phys. 10, 269 (1968)

22. Driessler, W. : On the type of local algebras in quantum field theory, Commun. Math. Phys. 59, 295 (1977)

23. Fröhlich, J. : New superselection sectors ("soliton states") in two dimensional Bose quantum field models, Commun. Math. Phys. 47, 269 (1976)

24. Borchers, H. J. : A remark on a theorem of B. Misra, Commun. Math. Phys. 4, 315 (1967)

25. Kadison, R. V. : The trace in finite operator algebras, Proc. Am. Math. Soc. 12, 973 (1961)

26. Borchers, H. J. : Local rings and the connection of spin with statistics, Commun. Math. Phys. 1, $281(1965)$

27. Araki, H. : On the algebra of all local observables. Prog. Theor. Phys. 32, 844 (1964)

28. Hepp, K. : In: Axiomatic Field Theory. Brandeis University New York, London, Paris: Gordon and Breach 1965

29. Buchholz, D., Fredenhagen, K. : (to be published)

30. Balian, R., Drouffe, J. M., Itzykson, C. : Gauge fields on a lattice II. Gauge-invariant Ising model, Phys. Rev. D11, 2098 (1974)

31. West, G. B. : Confinement in Quantum Chromodynamics, Phys. Rev. Lett. 46, 1365 (1981)

32. Aks, S. : Proof that scattering implies production in quantum field theory, J. Math. Phys. 6, $516(1965)$

33. Morpurgo, G. : In: Quarks and Leptons, Acta Phys. Austr. Suppl. XXI, 5 (1979)

Communicated by R. Haag

Received August 7, 1981 